\title{
Tetrazole Ring Substitution at Carbon and Boron Sites of the Cobalt Bis(dicarbollide) Ion Available via Dipolar Cycloadditions
}

\author{
Suzan El Anwar, ${ }^{1}$ Zdeňka Růžičková, ${ }^{2}$ Dmytro Bavol, ${ }^{1}$ Lukáš Fojt ${ }^{3}$ and Bohumír Grüner ${ }^{1 *}$ \\ ${ }^{1}$ Institute of Inorganic Chemistry of Czech Academy of Sciences, 25068 Řež, Czech Republic. \\ gruner@iic.cas.cz \\ ${ }^{2}$ Faculty of Chemical Technology, University of Pardubice, Studentská 95, 53210 Pardubice, \\ Czech Republic. \\ ${ }^{3}$ Institute of Biophysics, Academy of Sciences of the Czech Republic, v.v.i., Královopolská 135, \\ 61265 Brno, Czech Republic
}

Table of Contents

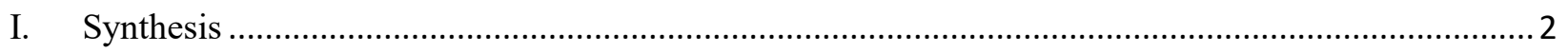

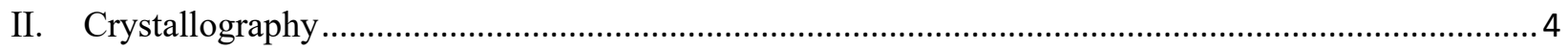

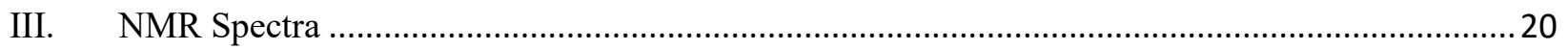

1. NMR spectra for nitrilium derivatives of cobalt bis(dicarbollide) ions ........................................20

2. NMR spectra for brominated derivatives of cobalt bis(dicabollide) ions ........................................2

3. NMR spectra for compounds containing tetrazole rings by dipolar cycloadditions ........................29

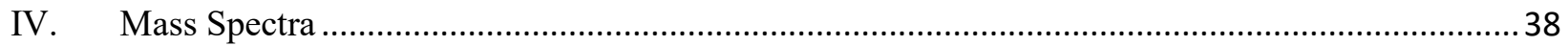

1. MS spectra for nitrilium derivatives of cobalt bis(dicarbollide) ions ...........................................38

2. MS spectra for brominated derivatives of cobalt bis(dicarbollide) ions ..........................................39

3. MS spectra for compounds containing tetrazole rings by dipolar cycloadditions ...........................40 


\section{Synthesis}

\section{Attempts to react organic azides with nitrile $3^{-}$and isonitrile I}

Reactions of nitrile $\mathbf{3}^{-}$and isonitrile $\mathbf{I}$ were tested with several organic azides 4(azidomethyl)benzonitrile and 1-azido acetic acid. However, no cyclic products resulted from those reactions and only addition products of proline formed in the reaction of isonitrile I with 1azido acetic acid.

For instance, the reaction of the nitrile $3^{-}$with 4-(azidomethyl)benzonitrile (1.5 eq) and Lproline, $150 \mathrm{~mol} \%$ gave no product, according to M.S. after stirring and heating at $130{ }^{\circ} \mathrm{C}$ for 4 days. The reaction of $\mathbf{I}$ with 1-azido acetic acid (1.8 eq.) gave after 2 days at rt. a product with composition corresponding to amide $\left[\left(8-\mathrm{CH}_{3} \mathrm{C}(\mathrm{O}) \mathrm{NH}-1,2-\mathrm{C}_{2} \mathrm{~B}_{9} \mathrm{H}_{10}\right)\left(1^{\prime}, 2^{\prime}-\mathrm{C}_{2} \mathrm{~B}_{9} \mathrm{H}_{11}\right)-3,3^{\prime}-\mathrm{Co}\right]^{-}$ (from MS) and only ca. $10 \%$ of the expected product, however after prolonged heating at $60{ }^{\circ} \mathrm{C}$, this was completely degraded and the molecular peak of the anticipated cyclic product disappeared in the MS. Adding a large excess of L-proline (10 eq.) activator to the reaction of $\mathbf{I}$ with 1-azido acetic acid (5 eq.) produced after 2 days at rt., a derivative of molecular mass $478.32(100 \%$ abundance, $\mathrm{m} / \mathrm{z}=482.32,10 \%)$ corresponding to composition [(8-N-(Proline)$\left.\left.\left(\mathrm{CH}_{3}\right) \mathrm{C}=\mathrm{NH}-1,2-\mathrm{C}_{2} \mathrm{~B}_{9} \mathrm{H}_{10}\right)\left(1^{\prime}, 2^{\prime}-\mathrm{C}_{2} \mathrm{~B}_{9} \mathrm{H}_{11}\right)-3,3^{\prime}-\mathrm{Co}\right]\left(\mathbf{1 2}^{-}\right)$. NMR spectra indicate that the proline was attached to carbon atom of the nitrile function, which is in line with previously prepared amidine derivatives. ${ }^{53}$ However, the ${ }^{1} \mathrm{H}$ and ${ }^{13} \mathrm{C}$ NMR spectra show that one couple of proton $\mathrm{CH}$ signals and both couples of carbon signals are split into two peaks. This indicated presence of diastereoisomers, tautomers or some H-bonding of proline molecule to the cage. Therefore, the assignment of the structure from NMR spectra remains still tentative. Unfortunately, crystals for $\mathrm{X}$-ray diffraction could not be grown. Amidines are known that may act as precursors in tetrazole synthesis using azides, ${ }^{66}$ however this particular reaction did not proceed further to the final stages of ring formation.

\section{[(8- (Proline-N)- $\left.\left(\mathrm{CH}_{3}\right) \mathrm{C}=\mathrm{NH}-1,2-\mathrm{C}_{2} \mathrm{~B}_{9} \mathrm{H}_{10}\right)\left(1^{\prime}, 2^{\prime}-\mathrm{C}_{2} \mathrm{~B}_{9} \mathrm{H}_{11}\right)-3,3$ '-Co] (12)}

[(8- $\left.\mathrm{NCCH}_{3}-1,2-\mathrm{C}_{2} \mathrm{~B}_{9} \mathrm{H}_{10}\right)\left(1\right.$ ', 2'- $\left.\mathrm{C}_{2} \mathrm{~B}_{9} \mathrm{H}_{11}\right)-3,3$ '-Co], I (0.207 g, $\left.0.569 \mathrm{mmol}\right)$ was dissolved in $25 \mathrm{~mL}$ of $\mathrm{CH}_{3} \mathrm{CN}$ and L-proline $(0.703 \mathrm{~g}, 6.106 \mathrm{mmol})$ was added. The reaction mixture was stirred at room temperature for 2 days. Then the excess of proline was removed by filtration and 
the filtrate was concentrated and purified on a silica gel column using $\mathrm{CH}_{2} \mathrm{Cl}_{2} / \mathrm{CH}_{3} \mathrm{CN}$ solvent mixture, gradually increasing the acetonitrile content from $20 \%$ to $100 \%$.

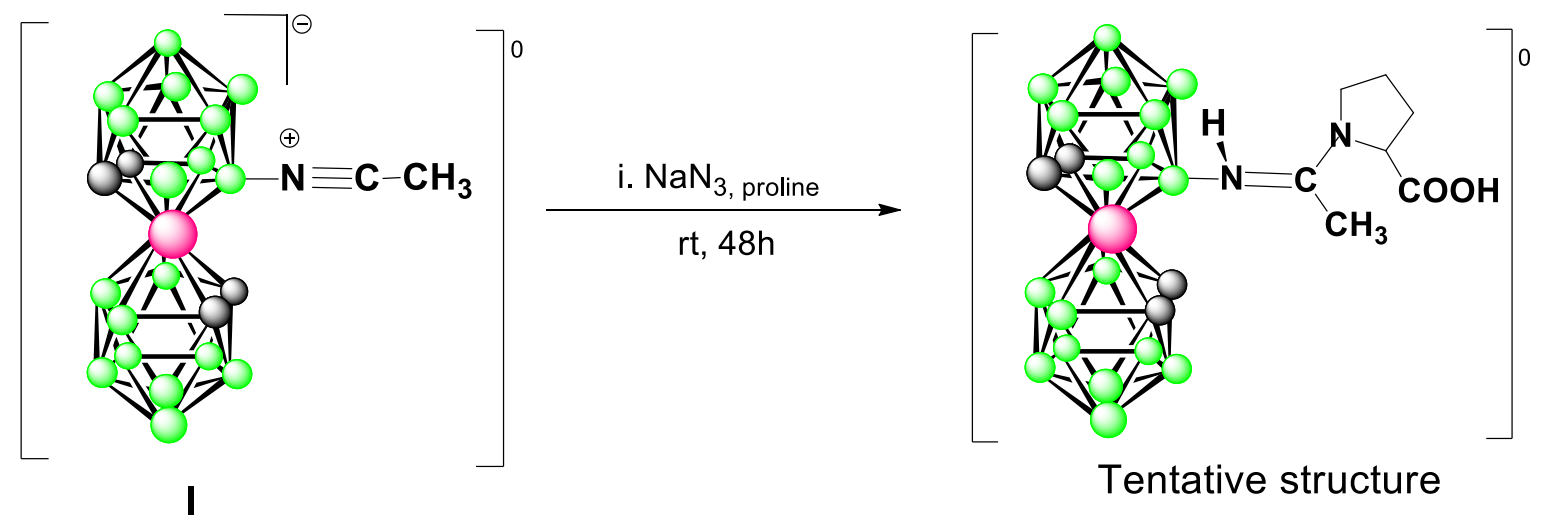

Scheme S1: Reaction leading to a proline substitution on the isonitrile group.

B(192 MHz; $\left.\mathrm{CD}_{3} \mathrm{CN} ; \mathrm{F}_{3} \mathrm{~B} . \mathrm{OEt}_{2}\right) 11.9$ (s, 1B, B8), 7.6 (br. d, 1B, B8'), 1.5 (d, 1B, B10', J= 100 Hz), -2.2 (d, 1B, B10, J=116 Hz), -5.4 (d, 2B, B4', 7'), -6.7 (d, 4B, B9', 12', B9, 12, J=131 Hz), 8.8 (d, 2B, B4, 7), -17.4 (br. d, 2B, B5'), -19.6 (br. d, 2B, B5, 11), -22.5 (br. d, 1B, B6'), -26.2 (br. d, 1B, B6); $\delta \mathrm{H}\left(600 \mathrm{MHz} ; \mathrm{CD}_{3} \mathrm{CN} ; \mathrm{Me}_{4} \mathrm{Si}\right) 6.67(1 \mathrm{H}, \mathrm{s}, 1 \mathrm{H}, \mathrm{NH}), 4.52(1 \mathrm{H}, \mathrm{s}, \mathrm{CH}-\mathrm{COOH})$, 3.96 (1H, s, CHcarborane), 3.39 (1H, s, CHcarborane), 3.86 (2H, s, CHcarborane), 3.35 (2H, m, $\left.\mathrm{CH}_{2}\right), 3.42\left(2 \mathrm{H}, \mathrm{m}, \mathrm{CH}_{2}\right), 2.19\left(2 \mathrm{H}, \mathrm{m}, \mathrm{CH} \mathrm{H}_{2}\right), 2.32(2 \mathrm{H}, \mathrm{s}, \mathrm{CH}) ; \delta \mathrm{C}\left(150 \mathrm{MHz} ; \mathrm{CD}_{3} \mathrm{CN}\right.$; $\left.\mathrm{Me}_{4} \mathrm{Si}\right) 172.8(\mathrm{COOH}), 163.6(C=\mathrm{N}), 52.5,52.3$ (CHcarborane), 49.1, $49.0(\mathrm{CH}$ carborane), 47.9 (CH-COOH), $30.3\left(\mathrm{CH}_{2}\right), 23.0\left(\mathrm{CH}_{2}\right), 21.6\left(\mathrm{CH}_{3}\right), 17.3\left(\mathrm{CH}_{2}\right) ; \mathrm{MS}\left(\mathrm{ESI}^{-}\right) \mathrm{m} / \mathrm{z}(\%)$ found: $\mathrm{MS}$ (ESI') $\mathrm{m} / \mathrm{z}$ (\%) found: 478.32 (100), 479.32 (80) [M-H]', calcd. 478.36 (100), 479.35 (79); 956.28 (40), 963.32 (1), 956.73 (100), 963.70 (1) [2M-H]'; 


\section{Crystallography}

Table S1: Experimental details for $2^{-} \mathrm{CH}_{2} \mathrm{Cl}_{2}$

Crystal data

Chemical formula

$M_{\mathrm{r}}$

Crystal system, space group

Temperature (K)

$a, b, c(\AA)$

$\beta\left(^{\circ}\right)$

$V\left(\AA^{3}\right)$

Z

Radiation type

$\mu\left(\mathrm{mm}^{-1}\right)$

Crystal size (mm)
$2\left(\mathrm{C}_{7} \mathrm{H}_{25} \mathrm{~B}_{18} \mathrm{CoN}\right) \cdot \mathrm{CH}_{2} \mathrm{Cl}_{2} \cdot 2\left(\mathrm{NC}_{4} \mathrm{H}_{12}\right)$

986.80

Monoclinic, $P 2_{1}$

150

$11.5778(6), 39.5902(18), 12.6906(6)$

$114.609(2)$

$5288.6(4)$

4

Mo $K \alpha$

0.76

$0.59 \times 0.30 \times 0.27$

Data collection

Diffractometer

Absorption correction

$T_{\min }, T_{\max }$

No. of measured, independent

and

observed $[I>2 \sigma(I)]$ reflections

$R_{\text {int }}$

0.058

$(\sin \theta / \lambda)_{\max }\left(\AA^{-1}\right)$

Refinement

$R\left[F^{2}>2 \sigma\left(F^{2}\right)\right], w R\left(F^{2}\right), S$

No. of reflections

No. of parameters

No. of restraints

$\mathrm{H}$-atom treatment

$\Delta \rho_{\max }, \Delta \rho_{\min }\left(\mathrm{e} \AA^{-3}\right)$

Absolute structure

Absolute structure parameter

0.650

23813

1249

2071

\section{Bruker D8 - Venture}

Multi-scan

SADABS2016/2 - Bruker AXS area detector scaling and absorption correction

$0.643,0.746$

$55052,23813,20364$

$0.067,0.170,1.06$

$\mathrm{H}$-atom parameters constrained

$w=1 /\left[\sigma^{2}\left(F_{\mathrm{o}}{ }^{2}\right)+(0.0692 P)^{2}+10.7351 P\right]$

where $P=\left(F_{\mathrm{o}}{ }^{2}+2 F_{\mathrm{c}}{ }^{2}\right) / 3$

$2.00,-0.95$

Refined as an inversion twin.

$0.36(2)$ 
Computer programs: Bruker Instrument Service vV6.2.3, APEX3 v2016.9-0 (Bruker AXS), SAINT V8.37A (Bruker AXS Inc., 2015), XT, VERSION 2014/5, SHELXL2017/1 (Sheldrick, 2017), Bruker SHELXTL.

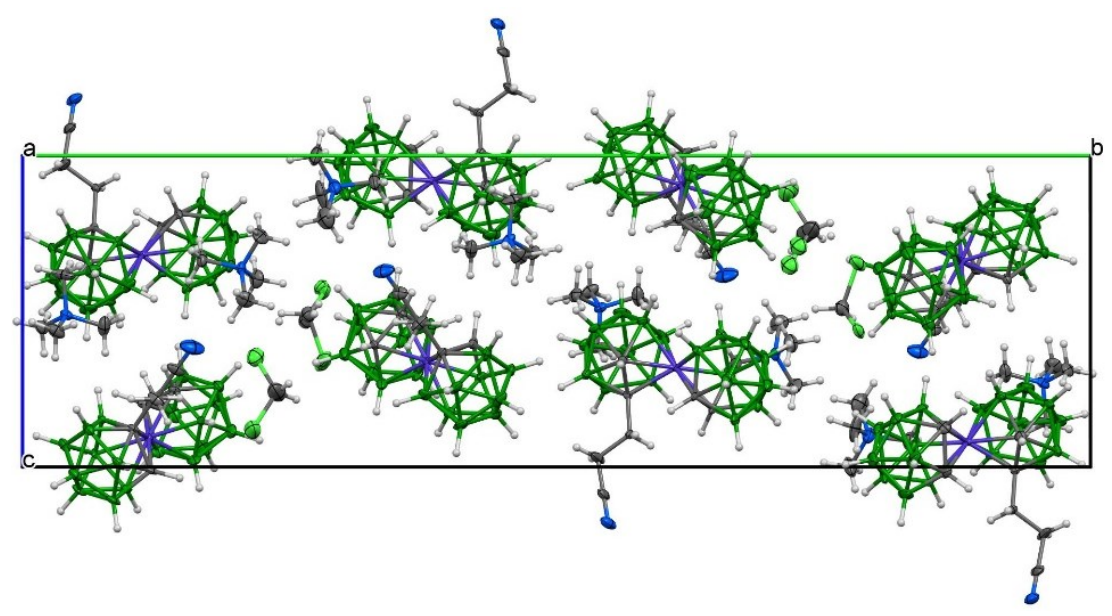

Figure S1: The unit cell of $\mathbf{2}^{-} \mathrm{CH}_{2} \mathrm{Cl}_{2}$ (ORTEP view, $40 \%$ probability level). 
Table S2: Experimental details for $\mathbf{2}^{-} \mathrm{CH}_{3} \mathrm{CN}$

\begin{tabular}{|c|c|}
\hline \multicolumn{2}{|l|}{ Crystal data } \\
\hline Chemical formula & $\mathrm{C}_{11} \mathrm{H}_{25} \mathrm{~B}_{18} \mathrm{CoN} \cdot \mathrm{C}_{2} \mathrm{H}_{3} \mathrm{~N} \cdot \mathrm{C}_{4} \mathrm{H}_{12} \mathrm{~N}$ \\
\hline$M_{\mathrm{r}}$ & 491.99 \\
\hline Crystal system, space group & Monoclinic, $P 2_{1} / n$ \\
\hline Temperature (K) & 150 \\
\hline$a, b, c(\AA)$ & $11.6500(8), 21.1820(12), 12.3326(8)$ \\
\hline$\beta\left(^{\circ}\right)$ & $117.489(2)$ \\
\hline$V\left(\AA^{3}\right)$ & $2699.7(3)$ \\
\hline$Z$ & 4 \\
\hline Radiation type & Mo $K \alpha$ \\
\hline$\mu\left(\mathrm{mm}^{-1}\right)$ & 0.65 \\
\hline Crystal size (mm) & $0.59 \times 0.58 \times 0.57$ \\
\hline \multicolumn{2}{|l|}{ Data collection } \\
\hline Diffractometer & Bruker D8 - Venture \\
\hline Absorption correction & $\begin{array}{l}\text { Multi-scan } \\
\text { SADABS2016/2 - Bruker AXS area detector scaling and absorption } \\
\text { correction }\end{array}$ \\
\hline$T_{\min }, T_{\max }$ & $0.370,0.746$ \\
\hline $\begin{array}{l}\text { No. of measured, independent } \\
\text { and } \\
\text { observed }[I>2 \sigma(I)] \text { reflections }\end{array}$ & $72594,6216,5358$ \\
\hline$R_{\text {int }}$ & 0.084 \\
\hline$(\sin \theta / \lambda)_{\max }\left(\AA^{-1}\right)$ & 0.651 \\
\hline \multicolumn{2}{|l|}{ Refinement } \\
\hline$R\left[F^{2}>2 \sigma\left(F^{2}\right)\right], w R\left(F^{2}\right), S$ & $0.037,0.083,1.08$ \\
\hline No. of reflections & 6216 \\
\hline No. of parameters & 405 \\
\hline $\mathrm{H}$-atom treatment & $\mathrm{H}$ atoms treated by a mixture of independent and constrained refinement \\
\hline$\Delta \rho_{\max }, \Delta \rho_{\min }\left(\mathrm{e} \AA^{-3}\right)$ & $0.39,-0.34$ \\
\hline
\end{tabular}

Computer programs: Bruker Instrument Service vV6.2.3, APEX3 v2016.9-0 (Bruker AXS), SAINT V8.37A (Bruker AXS Inc., 2015), XT, VERSION 2014/5, SHELXL2017/1 (Sheldrick, 2017), Bruker SHELXTL. 


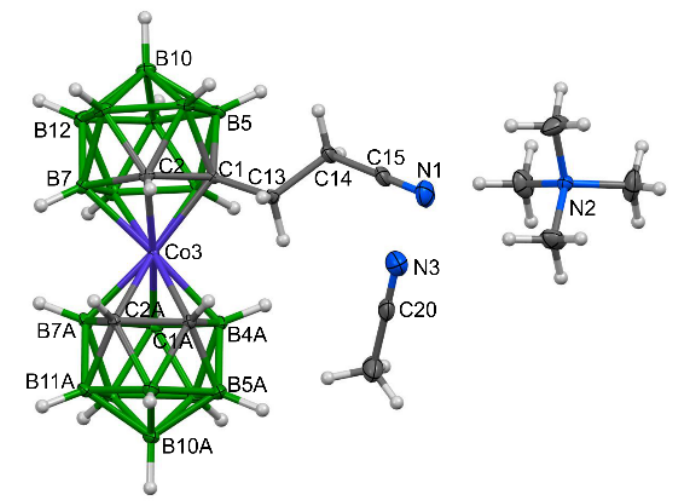

Figure S2: The molecular structure of $\mathrm{Me}_{4} \mathrm{N2}^{-} \cdot \mathrm{CH}_{3} \mathrm{CN}$ (ORTEP view, $40 \%$ probability level). Selected interatomic distances $[\AA]$ and angles $\left[{ }^{\circ}\right]: \mathrm{N} 1 \mathrm{C} 15$ 1.131(3), $\mathrm{C} 1 \mathrm{C} 13$ 1.536(2), C1 C2 1.631(2), C1 B5 1.719(2), C1 B4 1.724(2), C1 B6 1.741(2), C1 Co3 2.0983(15), C2A C1A 1.604(2), C2A B11A 1.694(2), C2A B7A 1.707(2), C2A B6A 1.728(2), C2A Co3 2.0539(16), C2 Co3 2.0608(15), Co3 B4 2.0814(18), Co3 B7 2.0837(18), Co3 B7A 2.0914(18), Co3 B4A 2.0953(19), Co3 B8 2.1026(19), Co3 B8A 2.1181(19), C13 C1 B4 126.63(14), C13 C1 C2 117.70(13), C13 C1 B5 118.88(13), C2 C1 B5 109.14(12), C2 C1 B4 109.09(12), B5 C1 B4 62.95(10), C13 C1 B6 110.60(13), C2 C1 B6 61.36(10), B4 C1 B6 113.50(12), C13 C1 Co3 112.40(10), B5 C1 Co3 121.69(10), B6 C1 Co3 122.57(10), C1A C2A B11A 111.30(13), C1A C2A B7A 111.90(13), B11A C2A B6A 61.85(11), B7A C2A B6A 115.68(13), B11A C2A Co3 125.23(11), B6A C2A Co3 125.66(11).

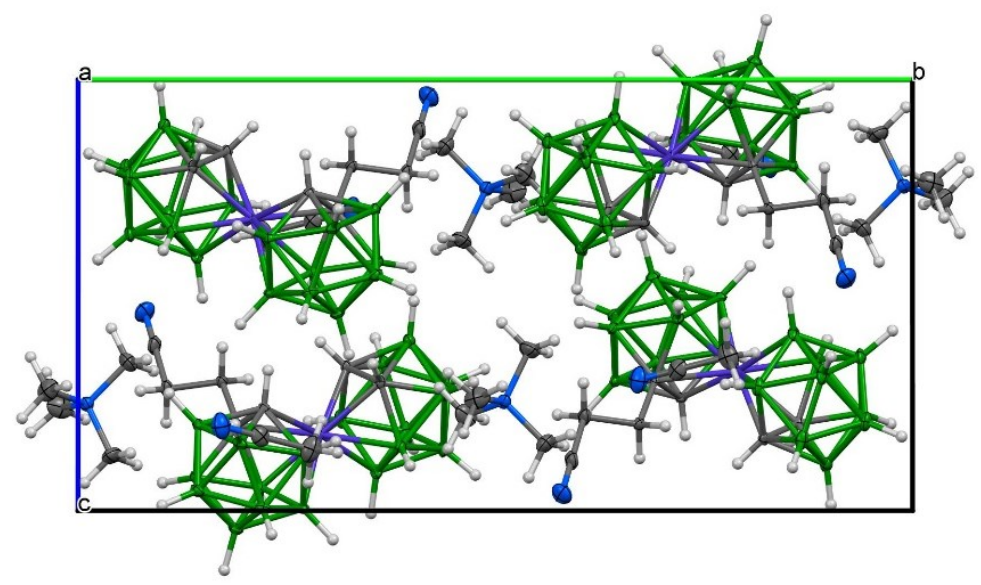

Figure S3: The unit cell of $2^{-} \mathrm{CH}_{3} \mathrm{CN}$ (ORTEP view, $40 \%$ probability level). 
Table S3: Experimental details for 3-

Crystal data

Chemical formula

$M_{\mathrm{r}}$

Crystal system, space group

Temperature $(\mathrm{K})$

$a, b, c(\AA)$

$\beta\left(^{\circ}\right)$

$V\left(\AA^{3}\right)$

Z

Radiation type

$\mu\left(\mathrm{mm}^{-1}\right)$

Crystal size (mm)
$\mathrm{C}_{8} \mathrm{H}_{27} \mathrm{~B}_{18} \mathrm{CoN} \cdot 0.5\left(\mathrm{CH}_{2} \mathrm{Cl}_{2}\right) \cdot \mathrm{C}_{4} \mathrm{H}_{12} \mathrm{~N}$

507.42

Monoclinic, $C 2 / c$

150

35.925 (2), 12.3252 (7), 12.6528 (7)

$101.439(3)$

$5491.2(6)$

8

Mo $K \alpha$

0.73

$0.44 \times 0.28 \times 0.11$

Data collection

Diffractometer

Absorption correction

$T_{\min }, T_{\max }$

No. of measured, independent

and

observed $[I>2 \sigma(I)]$ reflections

$R_{\text {int }}$

$(\sin \theta / \lambda)_{\max }\left(\AA^{-1}\right)$

Refinement

$R\left[F^{2}>2 \sigma\left(F^{2}\right)\right], w R\left(F^{2}\right), S$

No. of reflections

No. of parameters

No. of restraints

$\mathrm{H}$-atom treatment

$\Delta \rho_{\max }, \Delta \rho_{\min }\left(\mathrm{e} \AA^{-3}\right)$
Bruker D8 - Venture

Multi-scan

SADABS2016/2 - Bruker AXS area detector scaling and absorption correction

$0.436,0.746$

$82665,6301,5876$

0.071

0.652

$0.074,0.186,1.24$

6301

407

537

$\mathrm{H}$ atoms treated by a mixture of independent and constrained refinement $w=1 /\left[\sigma^{2}\left(F_{\mathrm{o}}^{2}\right)+(0.0424 P)^{2}+48.2489 P\right]$ where $P=\left(F_{\mathrm{o}}{ }^{2}+2 F_{\mathrm{c}}{ }^{2}\right) / 3$

$1.78,-1.54$

Computer programs: Bruker Instrument Service vV6.2.3, APEX3 v2016.9-0 (Bruker AXS), SAINT V8.37A (Bruker AXS Inc., 2015), XT, VERSION 2014/5, SHELXL2017/1 (Sheldrick, 2017), Bruker SHELXTL. 


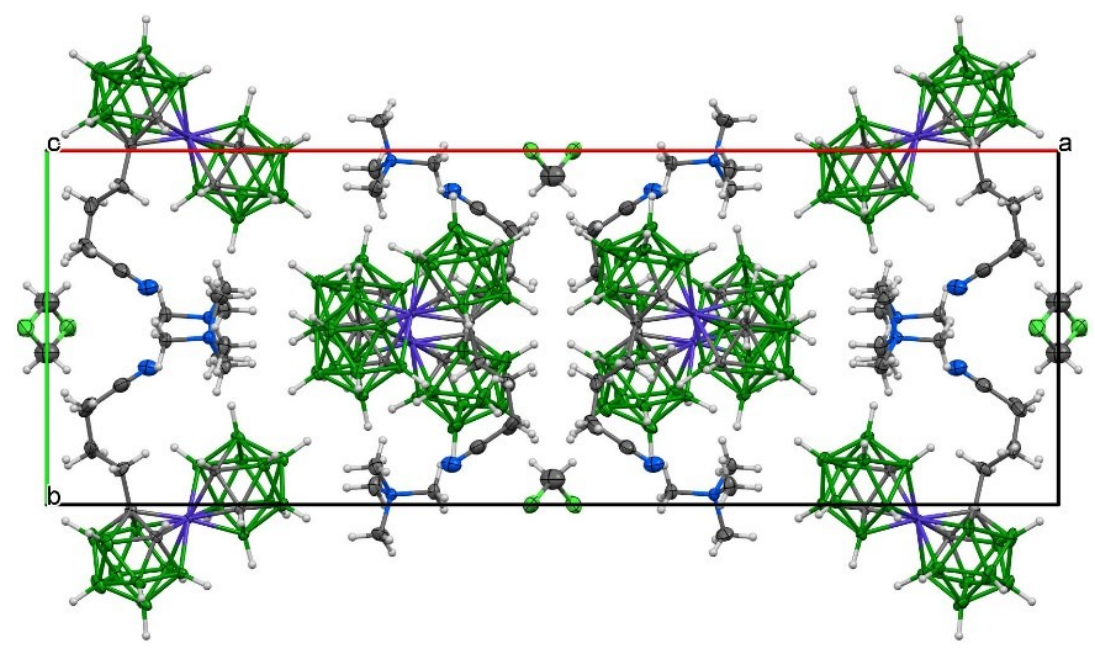

Figure S4: The unit cell of 3- (ORTEP view, 40\% probability level). 
Table S4: Experimental details for 4

Crystal data

\begin{tabular}{l|l}
\hline Chemical formula & $\mathrm{C}_{16} \mathrm{H}_{44} \mathrm{~B}_{18} \mathrm{Cl}_{4} \mathrm{CoN}_{3}$ \\
$M_{\mathrm{r}}$ & 673.85 \\
Crystal system, space group & Monoclinic, $P 2_{1} / c$ \\
Temperature (K) & 150 \\
$a, b, c(\AA)$ & $12.3099(7), 13.6660(7), 20.3456(10)$ \\
$\beta\left({ }^{\circ}\right)$ & $103.943(2)$ \\
$V\left(\AA^{3}\right)$ & $3321.8(3)$ \\
$Z$ & 4 \\
Radiation type & $\mathrm{Mo} \mathrm{K \alpha}$ \\
$\mu\left(\mathrm{mm}^{-1}\right)$ & 0.86 \\
Crystal size $(\mathrm{mm})$ & $0.40 \times 0.14 \times 0.10$ \\
\hline
\end{tabular}

Data collection

\begin{tabular}{l|l}
\hline Diffractometer & $\begin{array}{l}\text { Bruker D8 - Venture } \\
\text { Absorption correction }\end{array}$ \\
$T_{\min }, T_{\max }$ & $\begin{array}{l}\text { SADABS2016/2 - Bruker AXS area detector scaling and absorption } \\
\text { correction } \\
0.340,0.746\end{array}$ \\
No. of measured, independent & $62653,7622,6178$ \\
and & \\
observed $[I>2 \sigma(I)]$ reflections & \\
$R_{\text {int }}$ & 0.074 \\
$(\sin \theta / \lambda)_{\max }\left(\AA^{-1}\right)$ & 0.651
\end{tabular}

Refinement

\begin{tabular}{l|l}
\hline$R\left[F^{2}>2 \sigma\left(F^{2}\right)\right], w R\left(F^{2}\right), S$ & $0.064,0.183,1.07$
\end{tabular}

No. of reflections 7622

No. of parameters 479

No. of restraints 600

$\mathrm{H}$-atom treatment $\quad \mathrm{H}$ atoms treated by a mixture of independent and constrained refinement $\Delta \rho_{\max }, \Delta \rho_{\min }\left(\mathrm{e} \AA^{-3}\right) \quad 0.80,-1.37$

Computer programs: Bruker Instrument Service vV6.2.3, APEX3 v2016.9-0 (Bruker AXS), SAINT V8.37A (Bruker AXS Inc., 2015), XT, VERSION 2014/5, SHELXL2017/1 (Sheldrick, 2017), Bruker SHELXTL. 


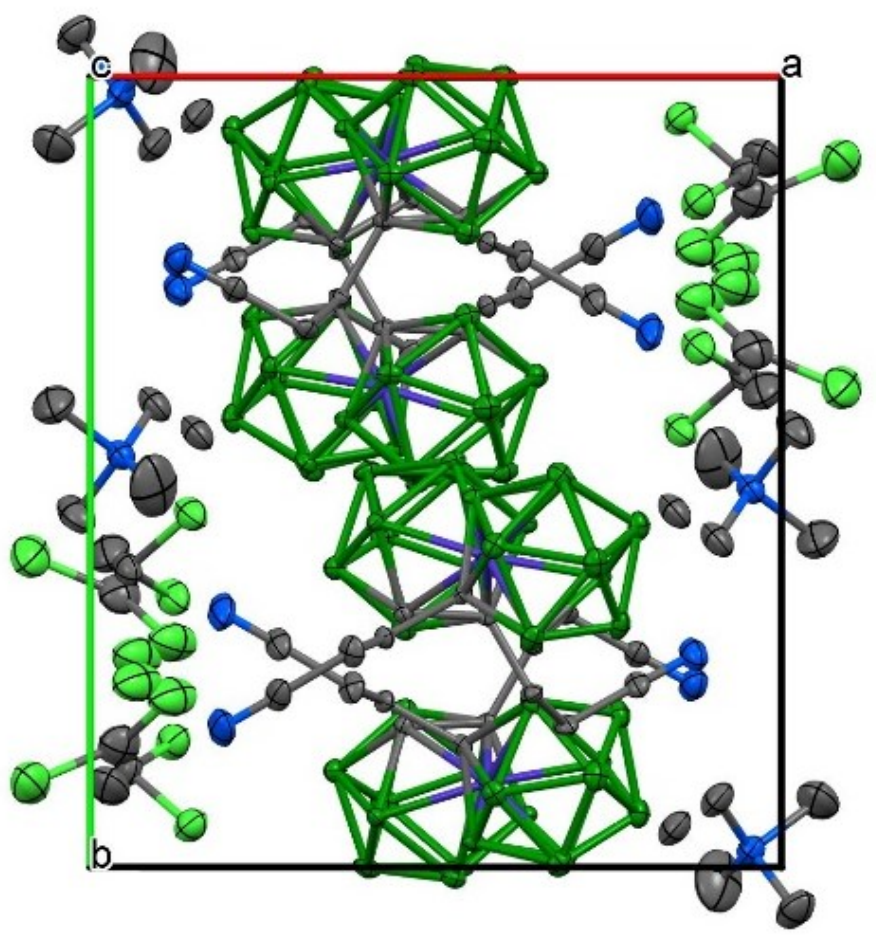

Figure S5: The unit cell of $\mathbf{4}^{-}$(ORTEP view, $40 \%$ probability level). 
Table S5: Experimental details for 5

Crystal data

Chemical formula

$M_{\mathrm{r}}$

\section{$\mathrm{C}_{4} \mathrm{H}_{21} \mathrm{~B}_{18} \mathrm{BrCoCs}$}

535.54

Crystal system, space group

Monoclinic, $P 2_{1} / n$

Temperature $(\mathrm{K})$

150

$a, b, c(\AA)$

7.0044 (3), 27.1761 (9), 10.5281 (4)

$\beta\left({ }^{\circ}\right)$

109.353 (1)

$V\left(\AA^{3}\right)$

$1890.81(13)$

Z

4

Radiation type

Mo $K \alpha$

$\mu\left(\mathrm{mm}^{-1}\right)$

4.90

Crystal size (mm)

$0.41 \times 0.24 \times 0.18$

Data collection

\begin{tabular}{|c|c|}
\hline \multirow{2}{*}{$\begin{array}{l}\text { Diffractometer } \\
\text { Absorption correction }\end{array}$} & Bruker D8 - Venture \\
\hline & $\begin{array}{l}\text { Multi-scan } \\
\text { SADABS2016/2 - Bruker AXS area detector scaling and absorption } \\
\text { correction }\end{array}$ \\
\hline$T_{\min }, T_{\max }$ & $0.318,0.746$ \\
\hline $\begin{array}{l}\text { No. of measured, independent } \\
\text { and } \\
\text { observed }[I>2 \sigma(I)] \text { reflections }\end{array}$ & $45650,4358,3860$ \\
\hline$R_{\text {int }}$ & 0.059 \\
\hline$(\sin \theta / \lambda)_{\max }\left(\AA^{-1}\right)$ & 0.651 \\
\hline \multicolumn{2}{|l|}{ Refinement } \\
\hline$R\left[F^{2}>2 \sigma\left(F^{2}\right)\right], w R\left(F^{2}\right), S$ & $0.046,0.119,1.06$ \\
\hline No. of reflections & 4358 \\
\hline No. of parameters & 226 \\
\hline No. of restraints & 480 \\
\hline $\mathrm{H}$-atom treatment & $\begin{array}{l}\text { H-atom parameters constrained } \\
w=1 /\left[\sigma^{2}\left(F_{\mathrm{o}}^{2}\right)+(0.0511 P)^{2}+15.1889 P\right] \\
\text { where } P=\left(F_{\mathrm{o}}^{2}+2 F_{\mathrm{c}}^{2}\right) / 3\end{array}$ \\
\hline$\Delta \rho_{\max }, \Delta \rho_{\min }\left(\mathrm{e} \AA^{-3}\right)$ & $3.04,-1.73$ \\
\hline
\end{tabular}

Computer programs: Bruker Instrument Service vV6.2.3, APEX3 v2016.9-0 (Bruker AXS), SAINT V8.37A (Bruker AXS Inc., 2015), XT, VERSION 2014/5, SHELXL2017/1 (Sheldrick, 2017), Bruker SHELXTL. 
Dipolar Cycloadditions to Azide Anions with iso-Nitrilium and Alkylnitrile Derivatives of Cobalt Bis(dicarbollide) Ions

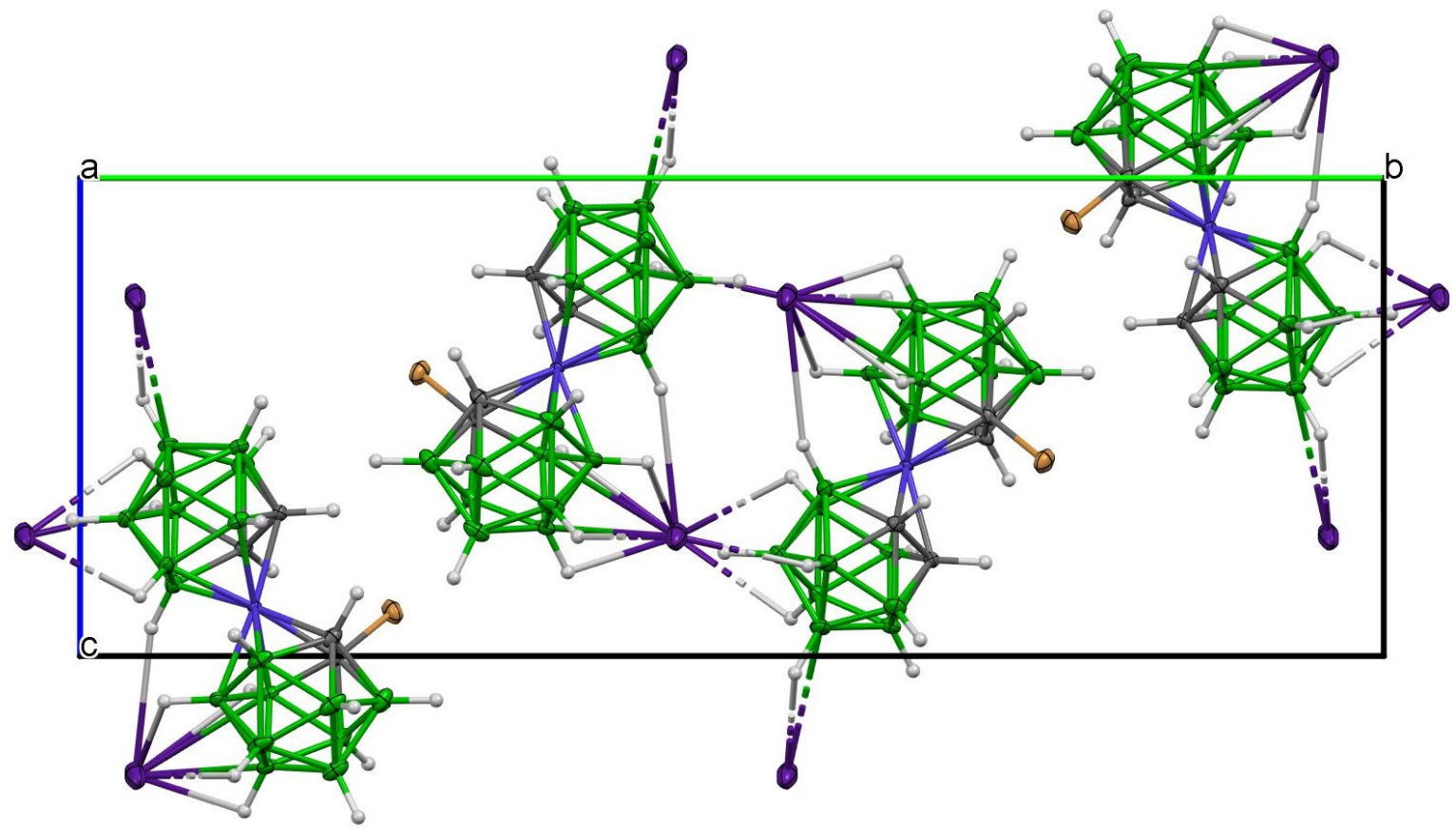

Figure S6: The unit cell of 5- (ORTEP view, 40\% probability level).

13 
Table S6: Experimental details for 6

Crystal data

Chemical formula

$M_{\mathrm{r}}$

$\mathrm{C}_{17} \mathrm{H}_{66} \mathrm{~B}_{36} \mathrm{Br}_{4} \mathrm{Cl}_{2} \mathrm{Co}_{2} \mathrm{~N}_{2}$

1196.27

Crystal system, space group

Monoclinic, $P 2_{1} / c$

Temperature $(\mathrm{K})$

150

$a, b, c(\AA)$

20.1121 (18), 18.911 (2), 13.4744 (14)

$\beta\left({ }^{\circ}\right)$

$99.416(3)$

$V\left(\AA^{3}\right)$

5055.9 (9)

$Z$

4

Radiation type

Mo $K \alpha$

$\mu\left(\mathrm{mm}^{-1}\right)$

3.95

Crystal size (mm)

$0.59 \times 0.44 \times 0.11$

Data collection

\begin{tabular}{|c|c|}
\hline Diffractometer & Bruker D8 - Venture \\
\hline Absorption correction & $\begin{array}{l}\text { Multi-scan } \\
\text { SADABS2016/2 - Bruker AXS area detector scaling and absorption } \\
\text { correction }\end{array}$ \\
\hline$T_{\min }, T_{\max }$ & $0.232,0.746$ \\
\hline $\begin{array}{l}\text { No. of measured, independent } \\
\text { and } \\
\text { observed }[I>2 \sigma(I)] \text { reflections }\end{array}$ & $119753,11640,7317$ \\
\hline$R_{\text {int }}$ & 0.213 \\
\hline$(\sin \theta / \lambda)_{\max }\left(\AA^{-1}\right)$ & 0.651 \\
\hline \multicolumn{2}{|l|}{ Refinement } \\
\hline$R\left[F^{2}>2 \sigma\left(F^{2}\right)\right], w R\left(F^{2}\right), S$ & $0.087,0.268,1.03$ \\
\hline No. of reflections & 11640 \\
\hline No. of parameters & 593 \\
\hline No. of restraints & 1043 \\
\hline $\mathrm{H}$-atom treatment & H-atom parameters constrained \\
\hline & $\begin{array}{l}w=1 /\left[\sigma^{2}\left(F_{\mathrm{o}}^{2}\right)+(0.1371 P)^{2}+48.2101 P\right] \\
\text { where } P=\left(F_{\mathrm{o}}^{2}+2 F_{\mathrm{c}}^{2}\right) / 3\end{array}$ \\
\hline$\Delta \rho_{\max }, \Delta \rho_{\min }\left(\mathrm{e} \AA^{-3}\right)$ & $1.57,-3.75$ \\
\hline
\end{tabular}

Computer programs: Bruker Instrument Service vV6.2.3, APEX3 v2016.9-0 (Bruker AXS), SAINT V8.37A (Bruker AXS Inc., 2015), XT, VERSION 2014/5, SHELXL2017/1 (Sheldrick, 2017), Bruker SHELXTL. 


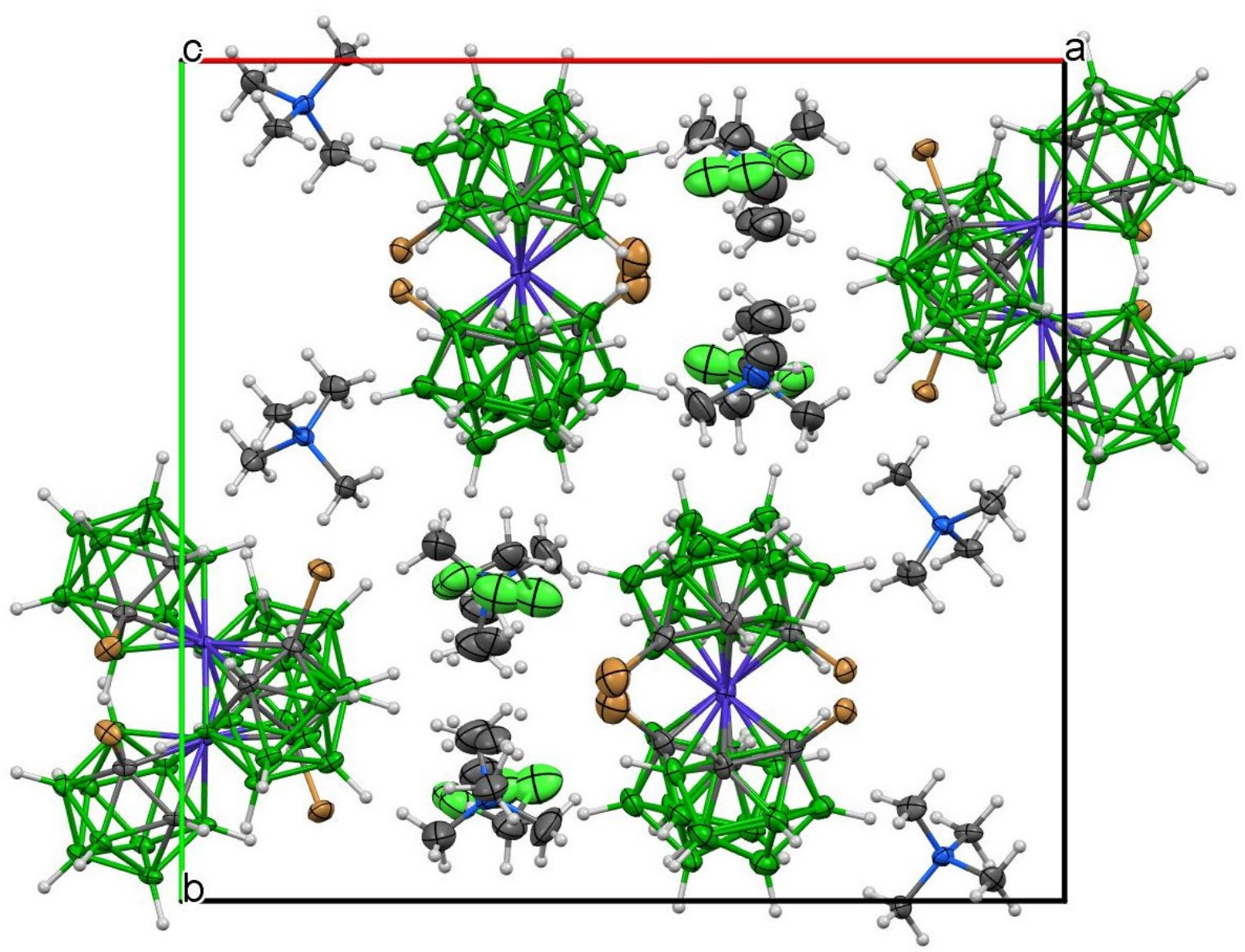

Figure S7: The unit cell of $6^{-}$(ORTEP view, 40\% probability level). 
Table S7: Experimental details for $7^{-}$

Crystal data

Chemical formula

$M_{\mathrm{r}}$

Crystal system, space group

Temperature $(\mathrm{K})$

$a, b, c(\AA)$

$\beta\left(^{\circ}\right)$

$V\left(\AA^{3}\right)$

$Z$

Radiation type

$\mu\left(\mathrm{mm}^{-1}\right)$

Crystal size (mm)

Data collection

Diffractometer

Absorption correction

$T_{\min }, T_{\max }$

No. of measured, independent and

observed $[I>2 \sigma(I)]$ reflections

$R_{\text {int }}$

$(\sin \theta / \lambda)_{\max }\left(\AA^{-1}\right)$

Refinement

$R\left[F^{2}>2 \sigma\left(F^{2}\right)\right], w R\left(F^{2}\right), S$

No. of reflections

No. of parameters

No. of restraints

$\mathrm{H}$-atom treatment

$\Delta \rho_{\max }, \Delta \rho_{\min }\left(\mathrm{e} \AA^{-3}\right)$
$\mathrm{C}_{9} \mathrm{H}_{34} \mathrm{~B}_{18} \mathrm{CoN}_{5}$

465.92

Monoclinic, $P 2_{1} / c$

150

14.6706 (14), 13.9561 (13), 12.8627 (14)

$114.894(3)$

$2388.9(4)$

4

Mo $K \alpha$

0.73

$0.52 \times 0.39 \times 0.24$
Bruker D8 - Venture

Multi-scan

SADABS2016/2 - Bruker AXS area detector scaling and absorption correction

$0.478,0.746$

$64964,5495,4587$

0.102

0.651

Computer programs: Bruker Instrument Service vV6.2.3, APEX3 v2016.9-0 (Bruker AXS), SAINT

V8.37A (Bruker AXS Inc., 2015), XT, VERSION 2014/5, SHELXL2017/1 (Sheldrick, 2017), Bruker SHELXTL. 


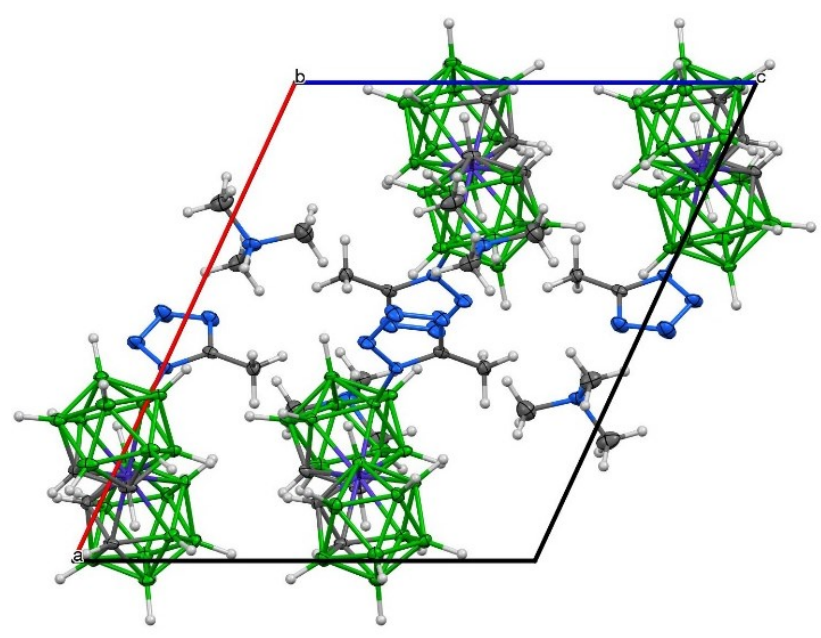

Figure S8: The unit cell of 7- (ORTEP view, 40\% probability level). 
Table S8: Experimental details for 10

\begin{tabular}{|c|c|}
\hline \multicolumn{2}{|l|}{ Crystal data } \\
\hline Chemical formula & $\mathrm{C}_{11} \mathrm{H}_{38} \mathrm{~B}_{18} \mathrm{CoN}_{5}$ \\
\hline$M_{\mathrm{r}}$ & 493.97 \\
\hline Crystal system, space group & Orthorhombic, $\mathrm{Pbca}$ \\
\hline Temperature (K) & 150 \\
\hline$a, b, c(\AA)$ & $15.8090(8), 10.2733(4), 31.5381$ (14) \\
\hline$V\left(\AA^{3}\right)$ & $5122.1(4)$ \\
\hline$Z$ & 8 \\
\hline Radiation type & Mo $K \alpha$ \\
\hline$\mu\left(\mathrm{mm}^{-1}\right)$ & 0.68 \\
\hline Crystal size (mm) & $0.50 \times 0.07 \times 0.07$ \\
\hline \multicolumn{2}{|l|}{ Data collection } \\
\hline Diffractometer & Bruker D8 - Venture \\
\hline Absorption correction & $\begin{array}{l}\text { Multi-scan } \\
S A D A B S 2016 / 2 \text { - Bruker AXS area detector scaling and absorption } \\
\text { correction }\end{array}$ \\
\hline$T_{\min }, T_{\max }$ & $0.500,0.746$ \\
\hline $\begin{array}{l}\text { No. of measured, independent } \\
\text { and } \\
\text { observed }[I>2 \sigma(I)] \text { reflections }\end{array}$ & $34177,5880,4562$ \\
\hline$R_{\text {int }}$ & 0.066 \\
\hline$(\sin \theta / \lambda)_{\max }\left(\AA^{-1}\right)$ & 0.651 \\
\hline \multicolumn{2}{|l|}{ Refinement } \\
\hline$R\left[F^{2}>2 \sigma\left(F^{2}\right)\right], w R\left(F^{2}\right), S$ & $0.064,0.120,1.12$ \\
\hline No. of reflections & 5880 \\
\hline No. of parameters & 336 \\
\hline No. of restraints & 543 \\
\hline H-atom treatment & $\begin{array}{l}\text { H atoms treated by a mixture of independent and constrained refinement } \\
w=1 /\left[\sigma^{2}\left(F_{\mathrm{o}}{ }^{2}\right)+(0.0225 P)^{2}+13.367 P\right] \\
\text { where } P=\left(F_{\mathrm{o}}{ }^{2}+2 F_{\mathrm{c}}{ }^{2}\right) / 3\end{array}$ \\
\hline$\Delta \rho_{\max }, \Delta \rho_{\min }\left(\mathrm{e} \AA^{-3}\right)$ & $0.50,-0.70$ \\
\hline
\end{tabular}

Computer programs: Bruker Instrument Service vV6.2.3, APEX3 v2016.9-0 (Bruker AXS), SAINT V8.37A (Bruker AXS Inc., 2015), XT, VERSION 2014/5, SHELXL2017/1 (Sheldrick, 2017), Bruker SHELXTL. 


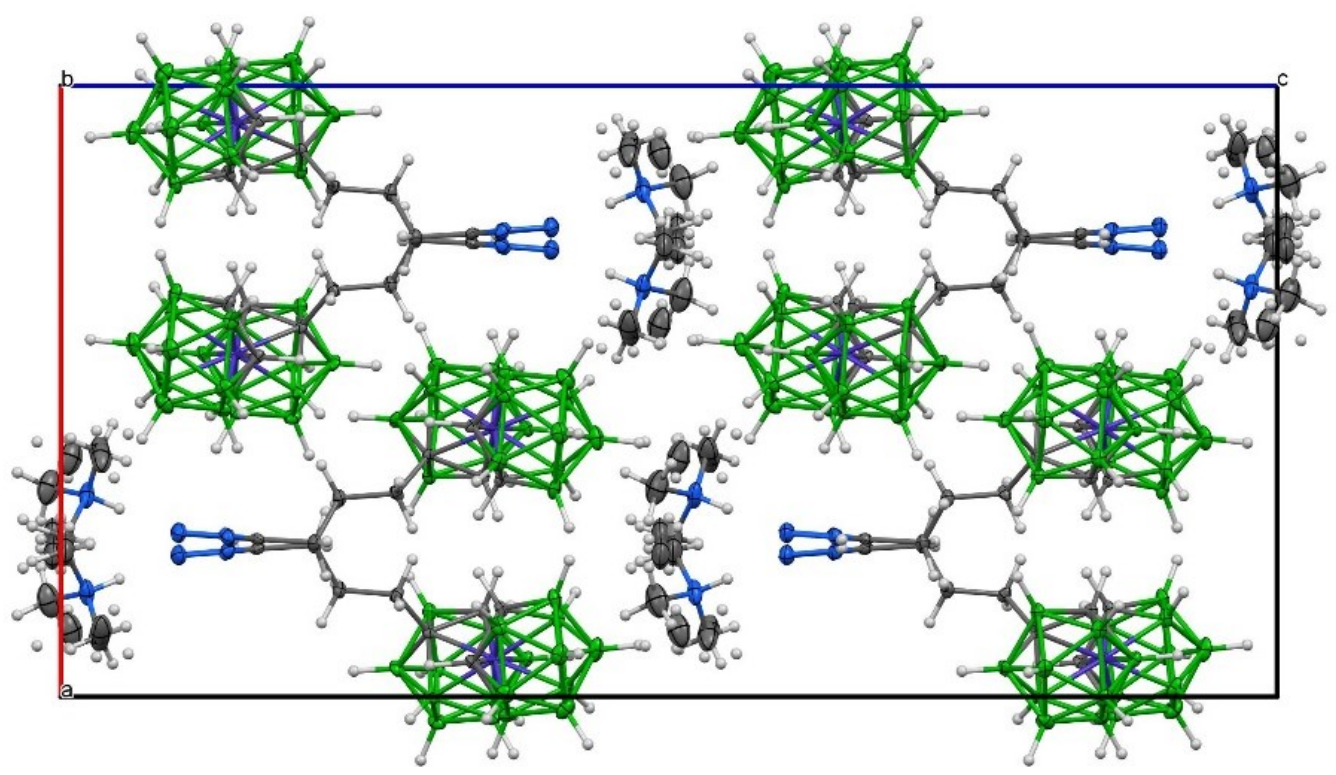

Figure S9: The unit cell of $\mathbf{1 0}^{-}$(ORTEP view, $40 \%$ probability level). 


\section{NMR Spectra}

1. NMR spectra for nitrilium derivatives of cobalt bis(dicarbollide) ions

a)

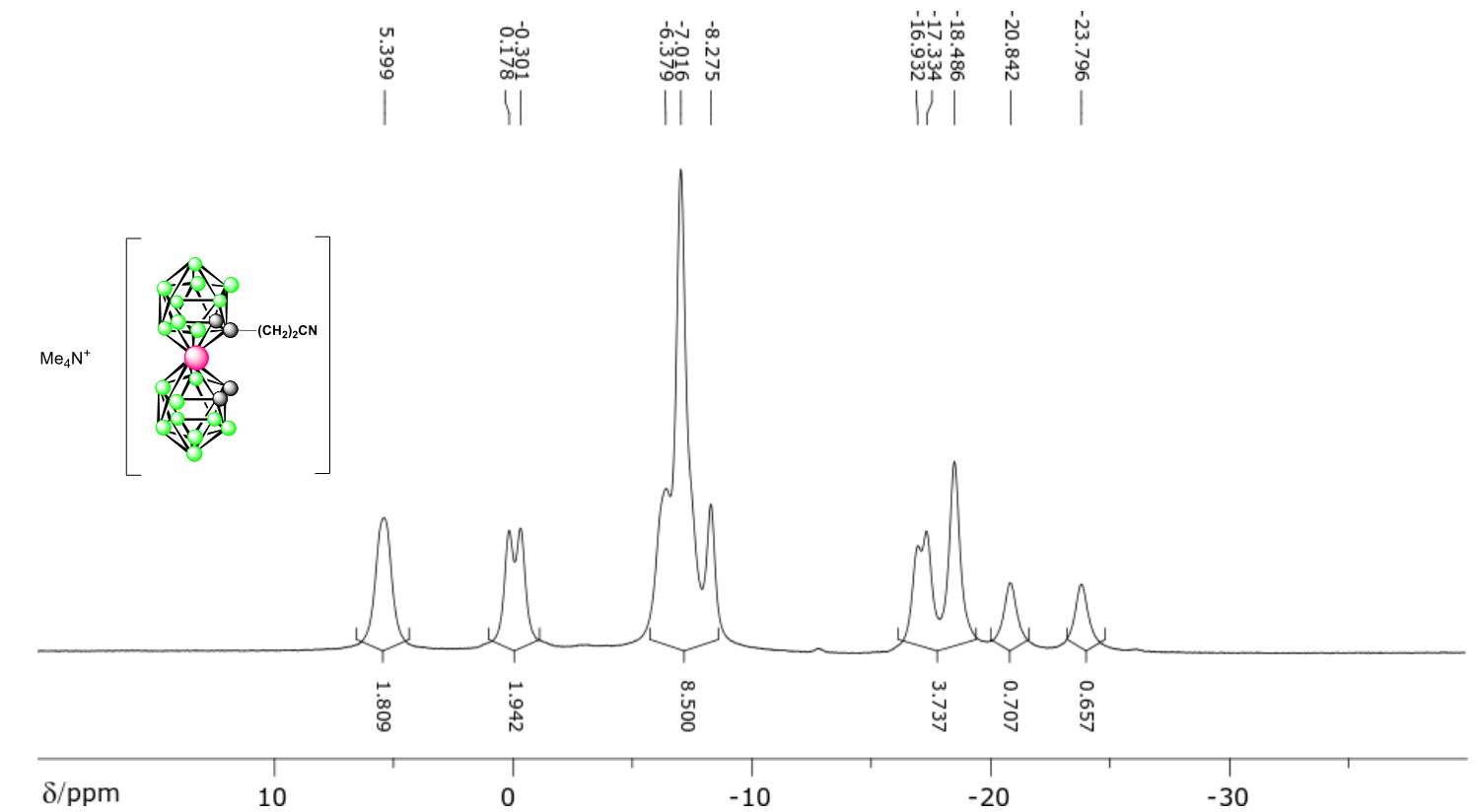

b)

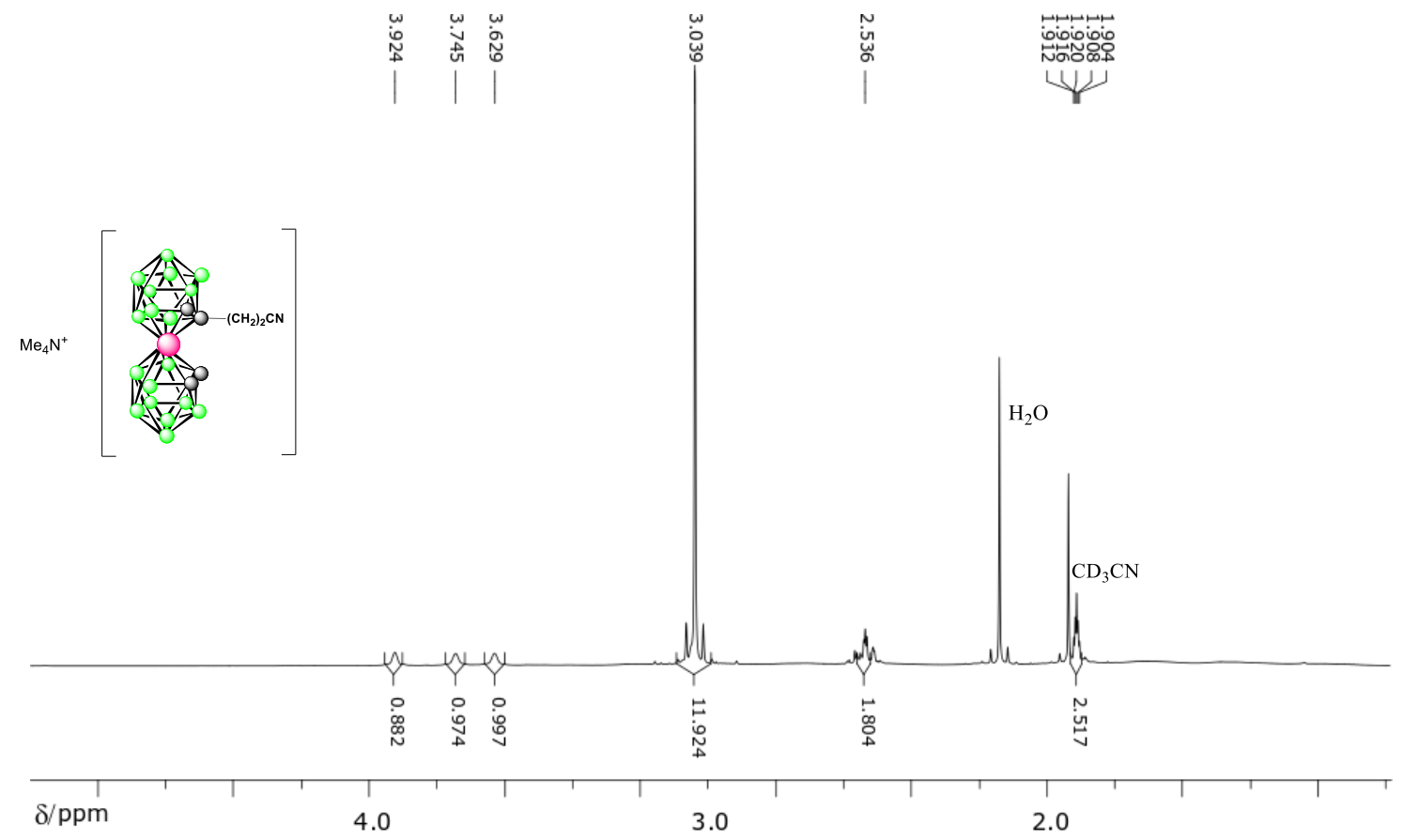


c)

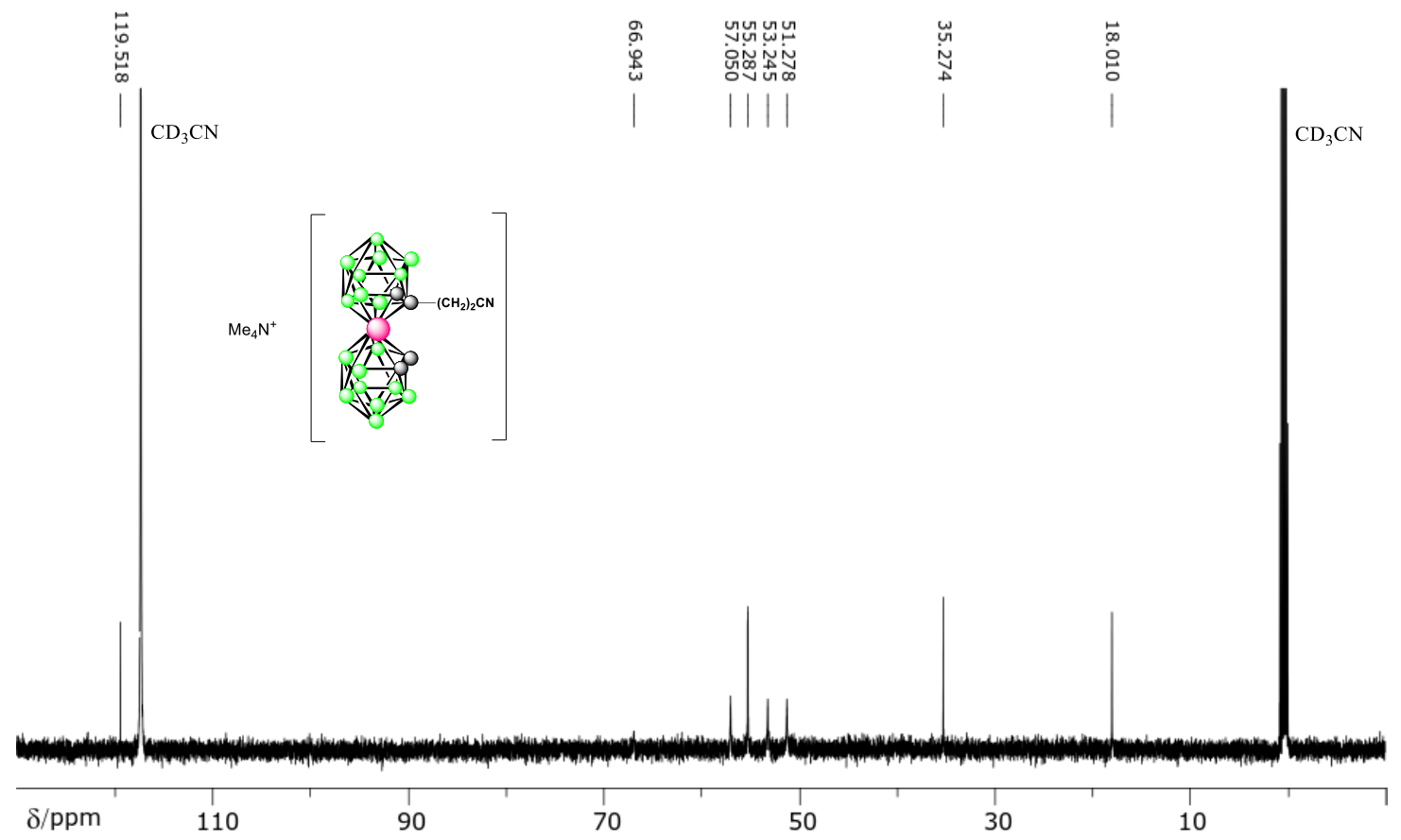

Figure S10. NMR spectra of compound $2^{-}$in $\mathrm{CD}_{3} \mathrm{CN}$, from top to bottom: a) $\left.{ }^{11} \mathrm{~B}\left\{{ }^{1} \mathrm{H}\right\} \mathrm{NMR}(192 \mathrm{MHz}), \mathrm{b}\right){ }^{1} \mathrm{H} \mathrm{NMR}(600 \mathrm{MHz})$, c) ${ }^{13} \mathrm{C}$ NMR $(150 \mathrm{MHz})$

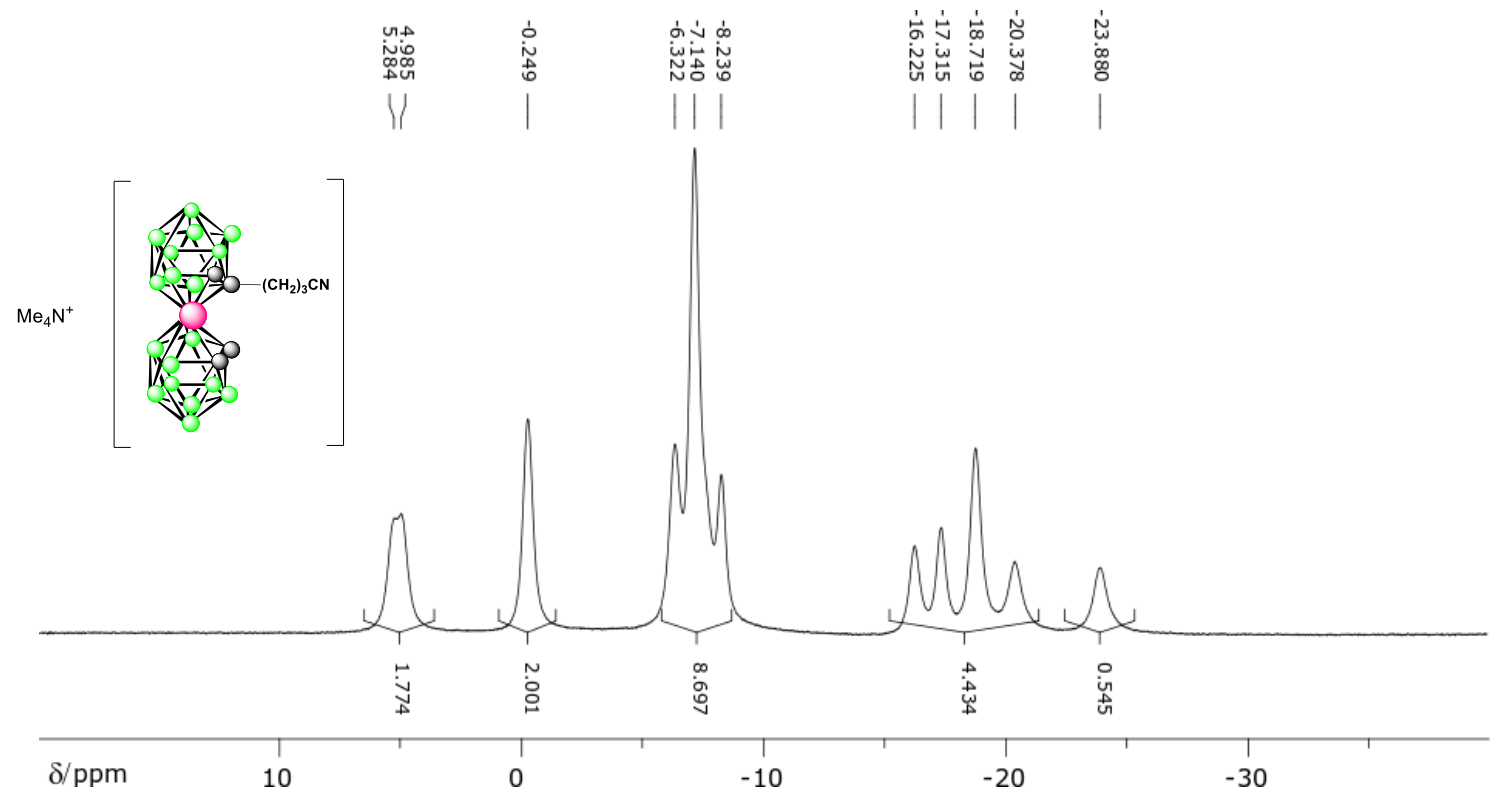


b)

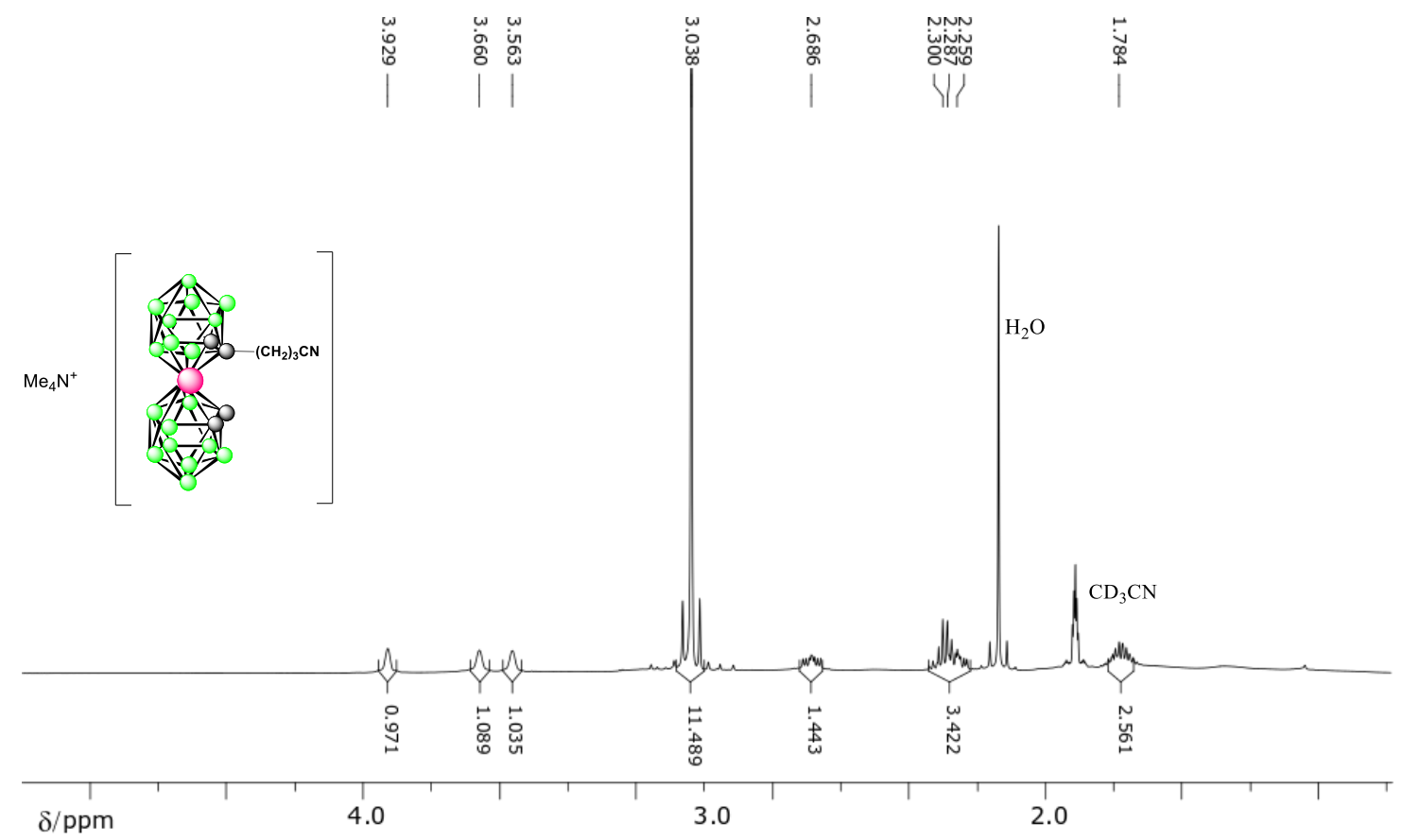

c)

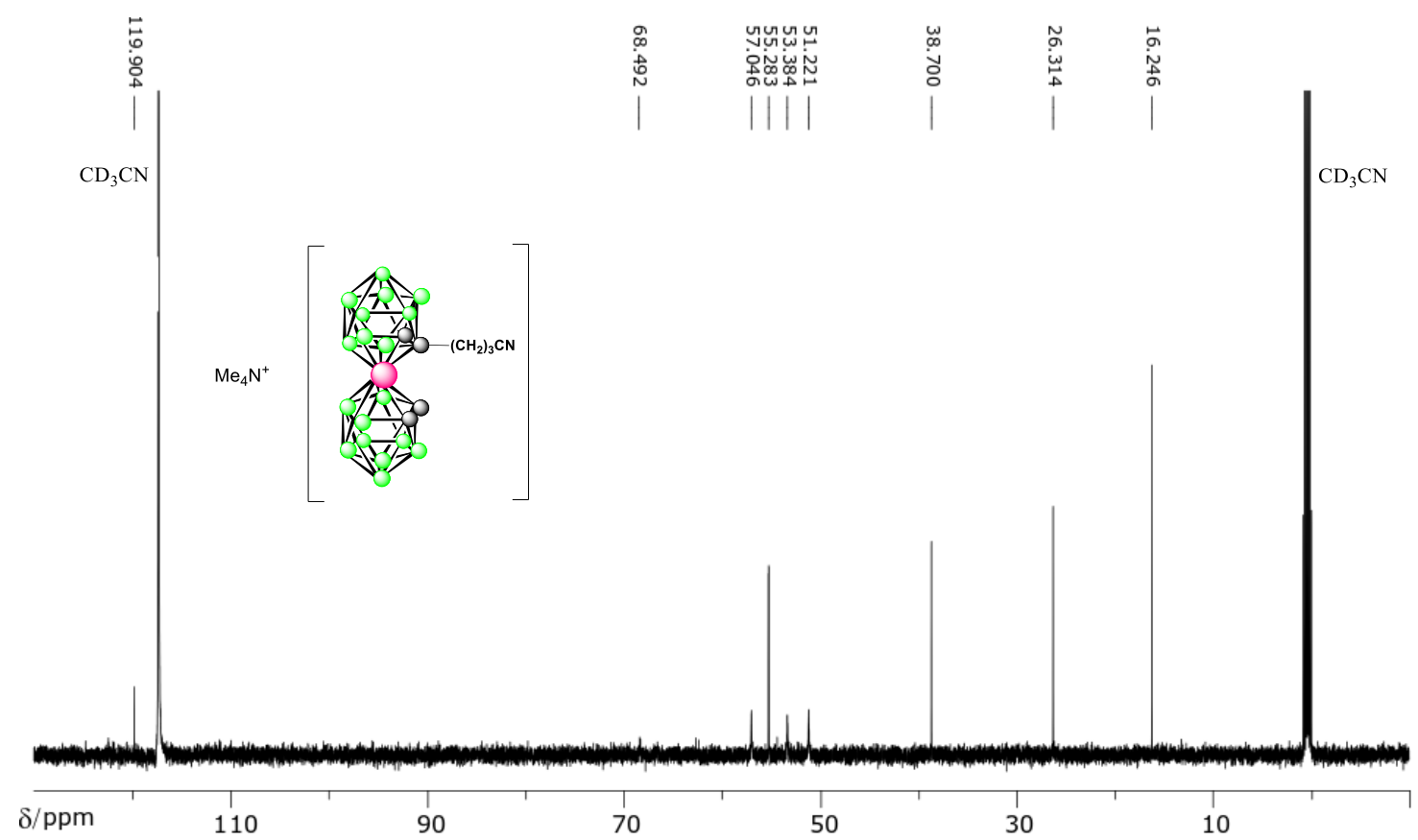

Figure S11. NMR spectra of compound $3^{-}$in $\mathrm{CD}_{3} \mathrm{CN}$, from top to bottom: a) $\left.{ }^{11} \mathrm{~B}\left\{{ }^{1} \mathrm{H}\right\} \mathrm{NMR}(192 \mathrm{MHz}), \mathrm{b}\right){ }^{1} \mathrm{H} \mathrm{NMR}(600 \mathrm{MHz})$, c) ${ }^{13} \mathrm{C}$ NMR $(150 \mathrm{MHz})$ 
a)

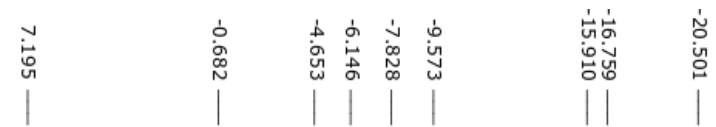

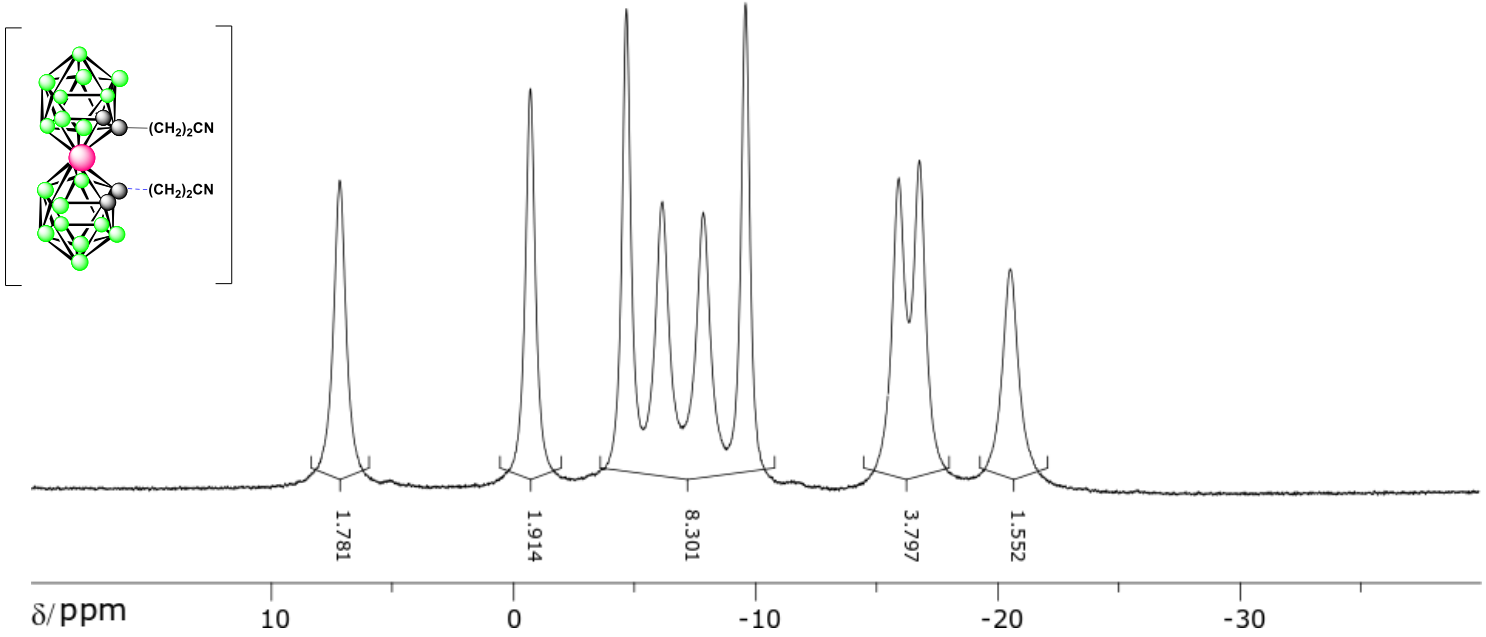

b)

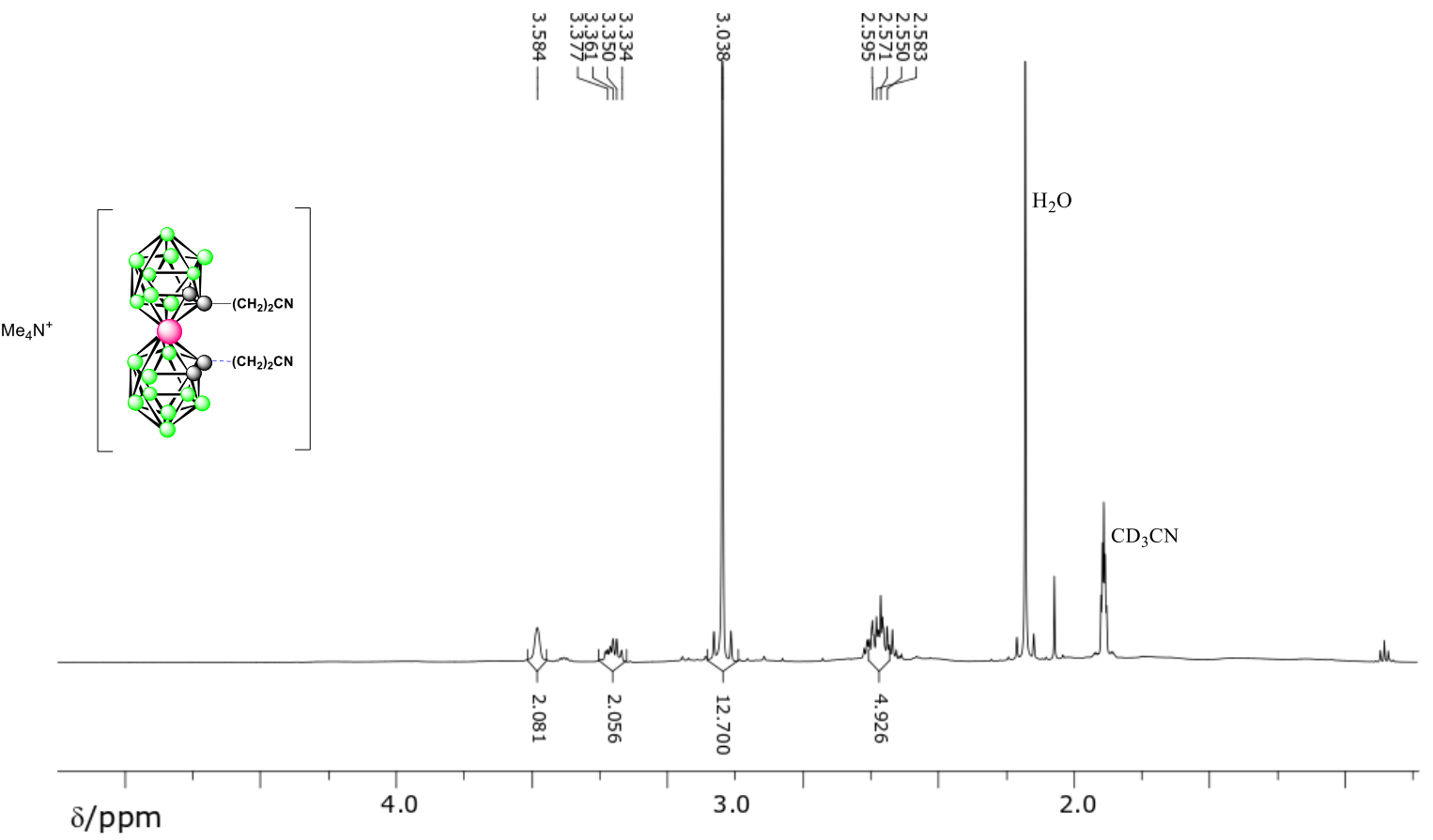


c)

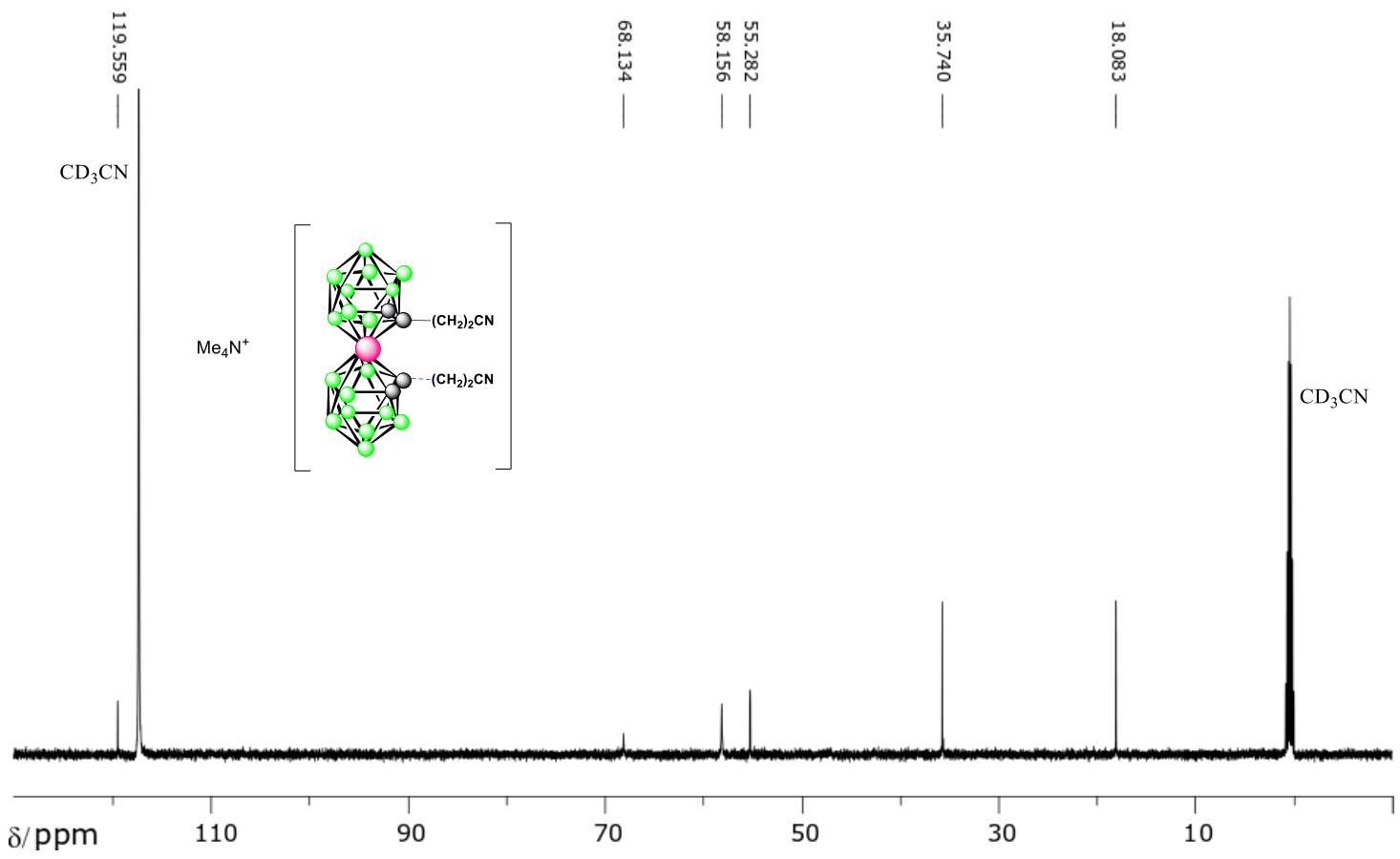

Figure S12. NMR spectra of compound $r a c-4^{-}$in $\mathrm{CD}_{3} \mathrm{CN}$, from top to bottom: a) $\left.{ }^{11} \mathrm{~B}\left\{{ }^{1} \mathrm{H}\right\} \mathrm{NMR}(192 \mathrm{MHz}), \mathrm{b}\right){ }^{1} \mathrm{H} \mathrm{NMR}(600$ $\mathrm{MHz}), \mathrm{c}){ }^{13} \mathrm{C}$ NMR $(150 \mathrm{MHz})$

2. NMR spectra for brominated derivatives of cobalt bis(dicabollide) ions

a)
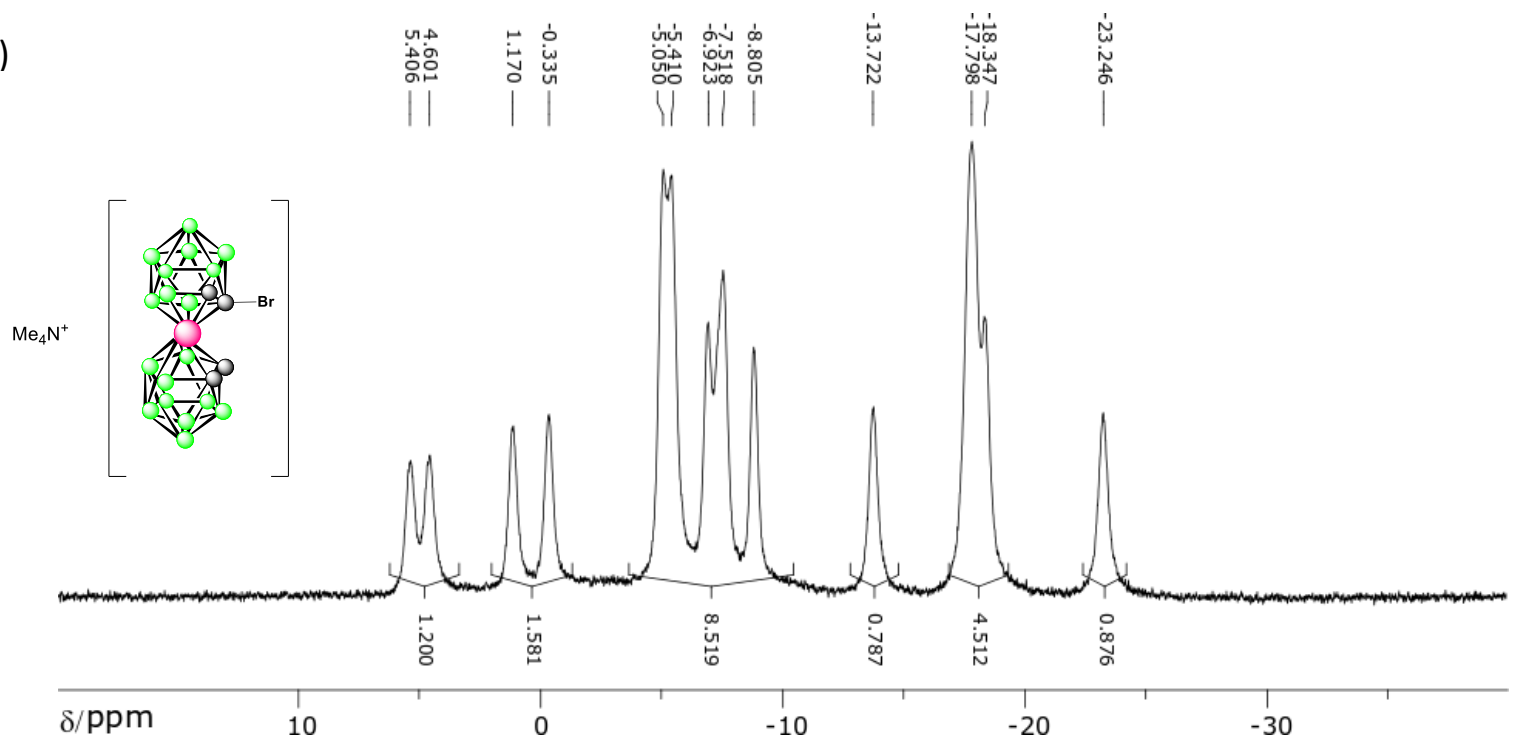
b)

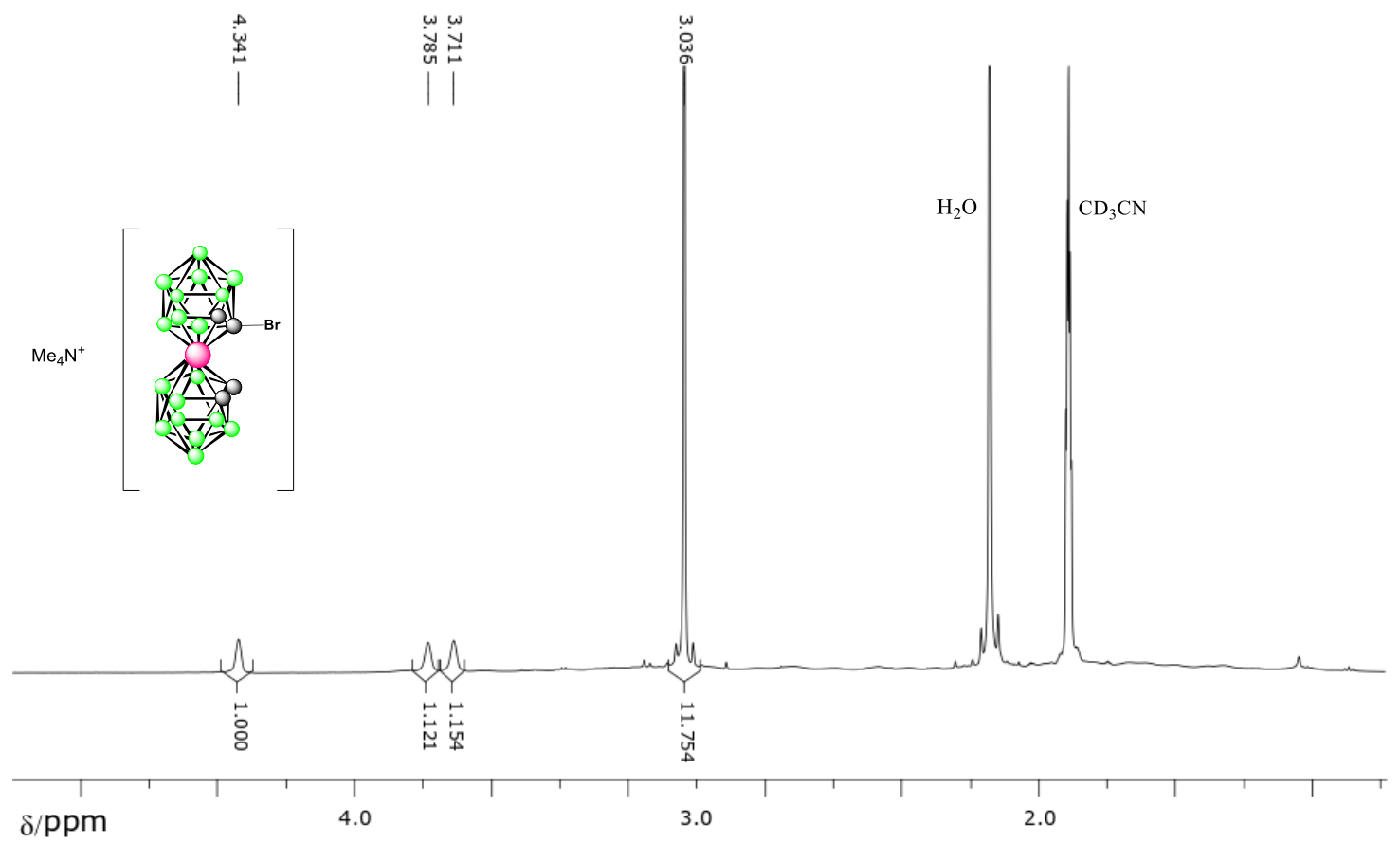

c)

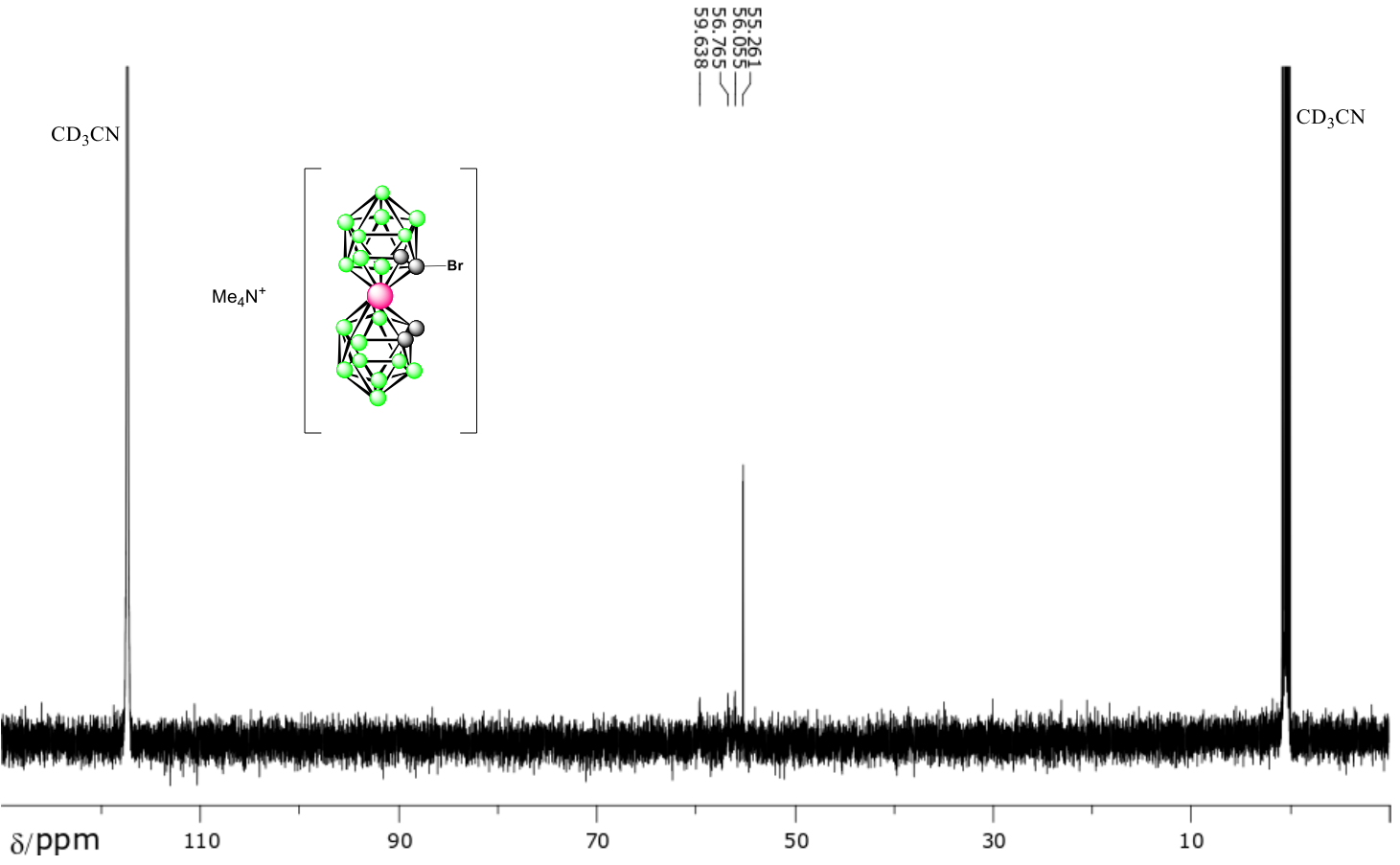

Figure S13. NMR spectra of compound $\mathbf{5}^{-}$in $\mathrm{CD}_{3} \mathrm{CN}$, from top to bottom: a) $\left.{ }^{11} \mathrm{~B}\left\{{ }^{1} \mathrm{H}\right\} \mathrm{NMR}(192 \mathrm{MHz}), \mathrm{b}\right){ }^{1} \mathrm{H} \mathrm{NMR}(600 \mathrm{MHz})$, c) ${ }^{13} \mathrm{C}$ NMR $(150 \mathrm{MHz})$ 
a)

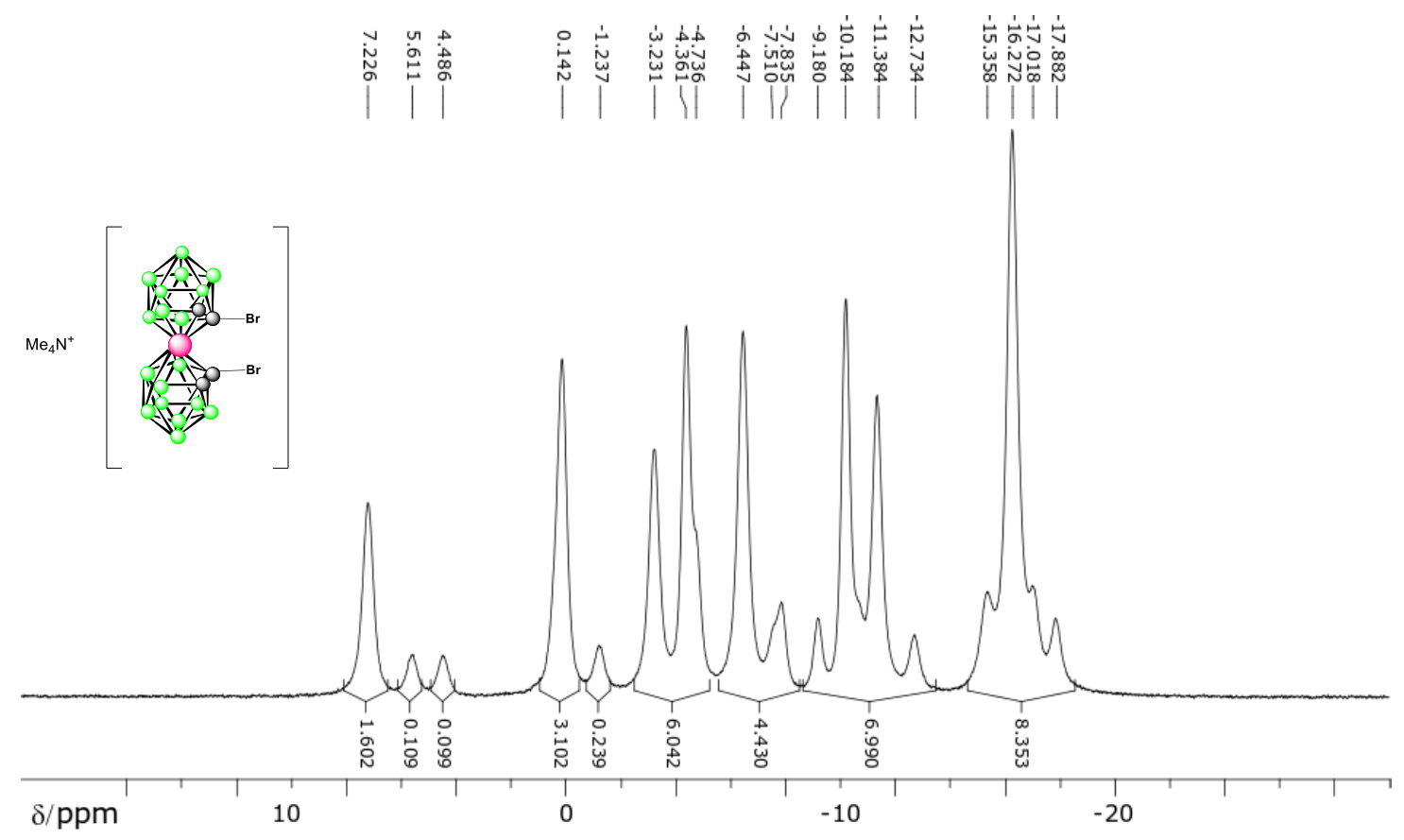

b)

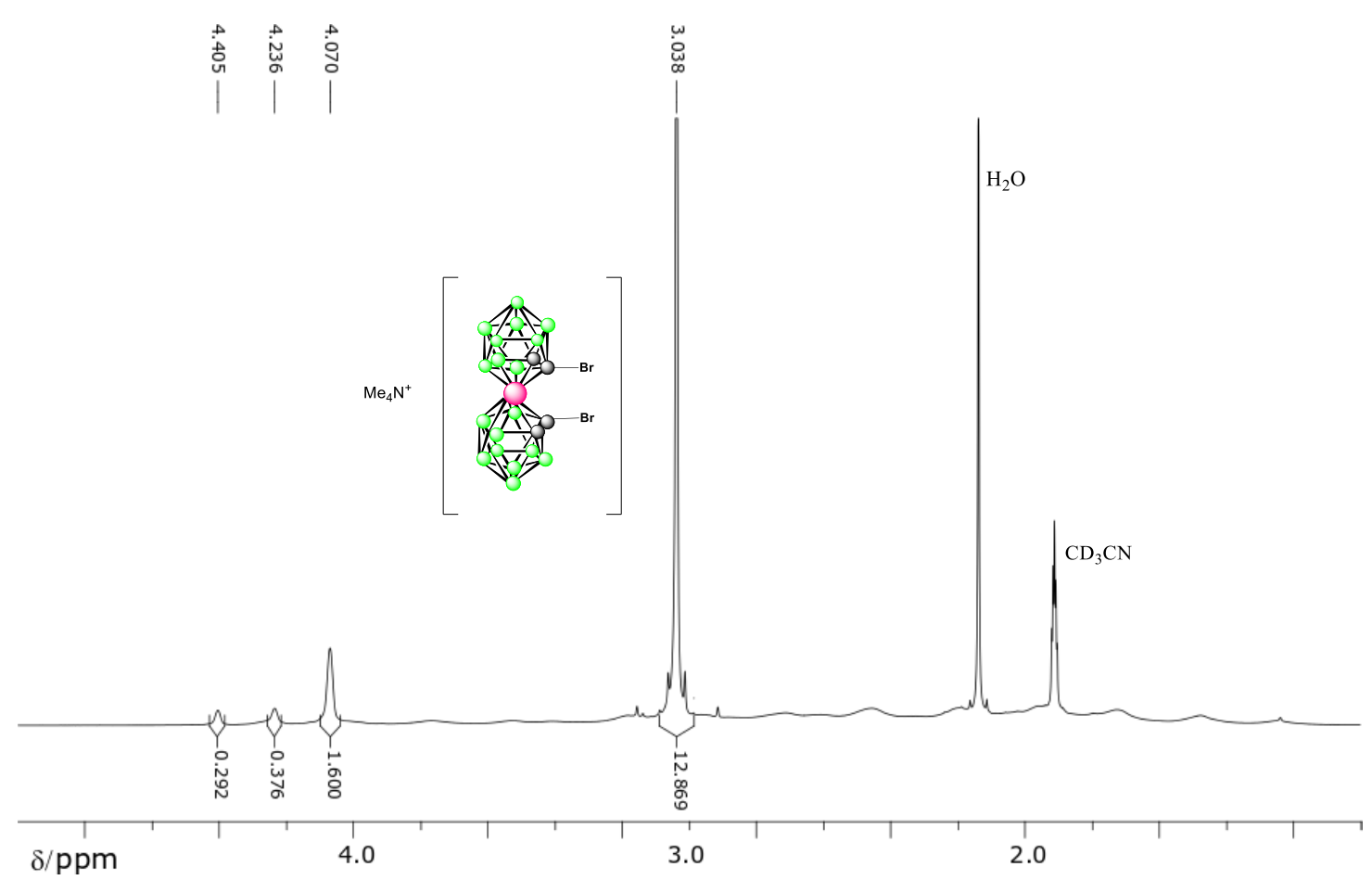


c)

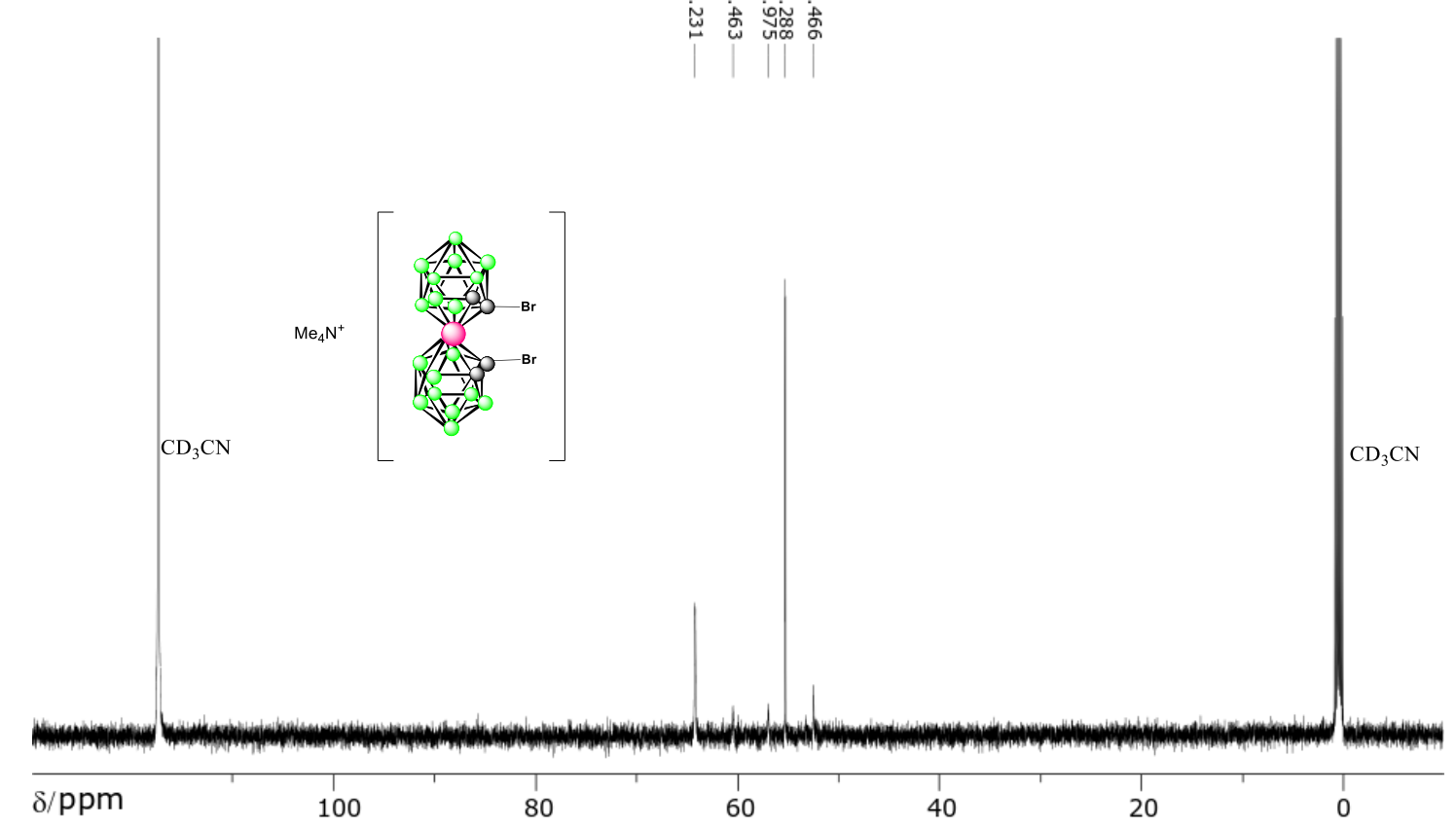

d)

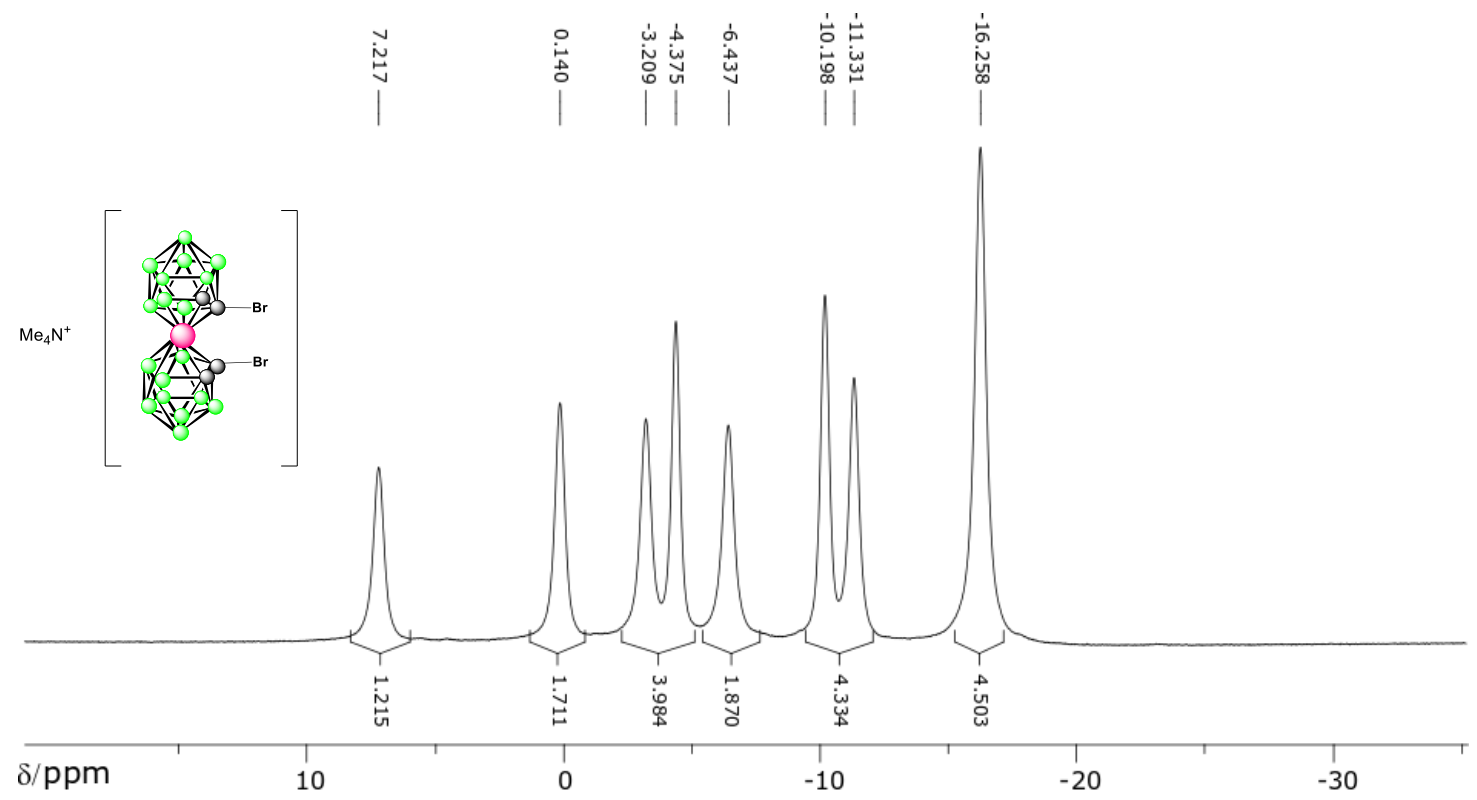


e)

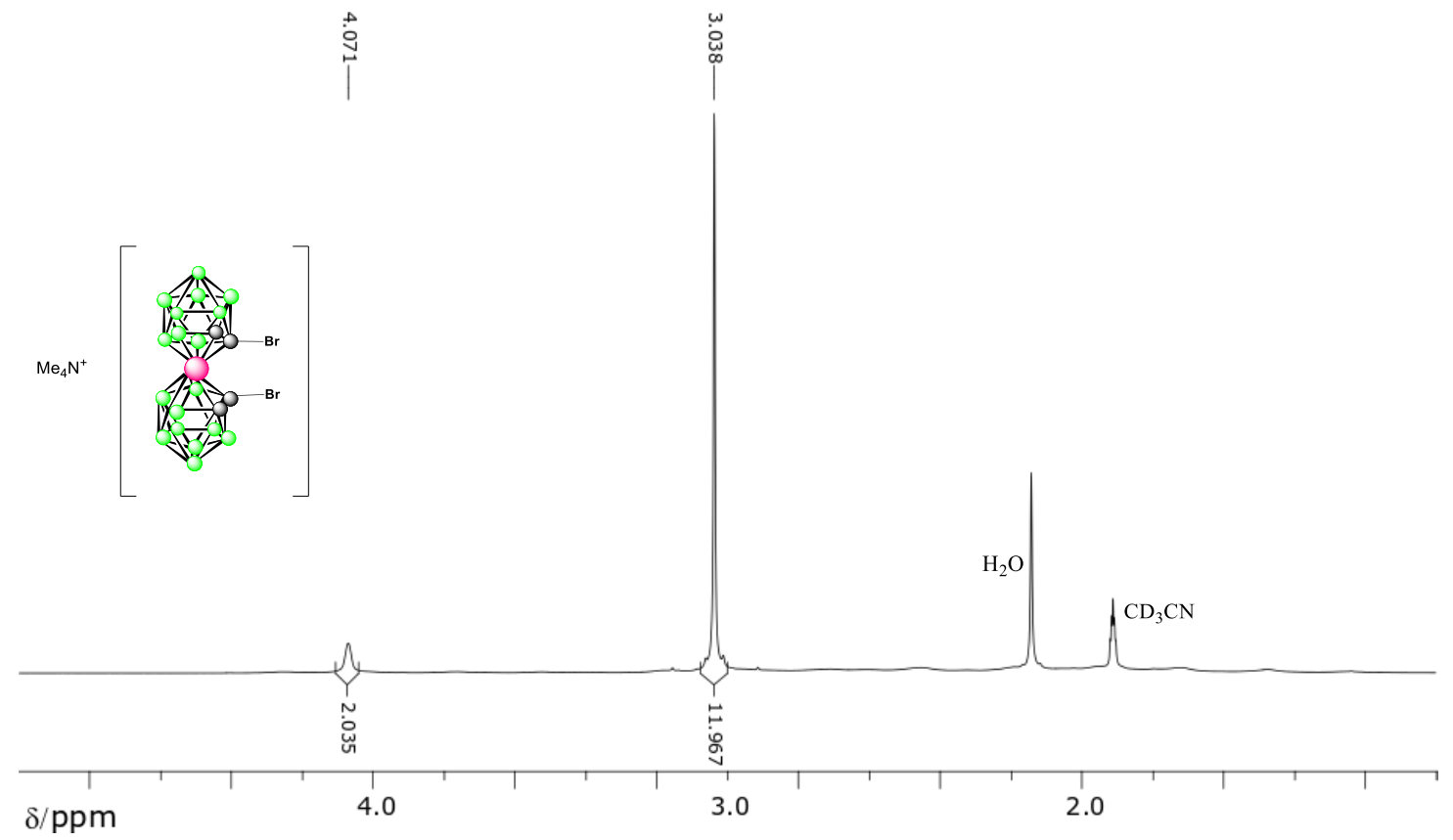

f)

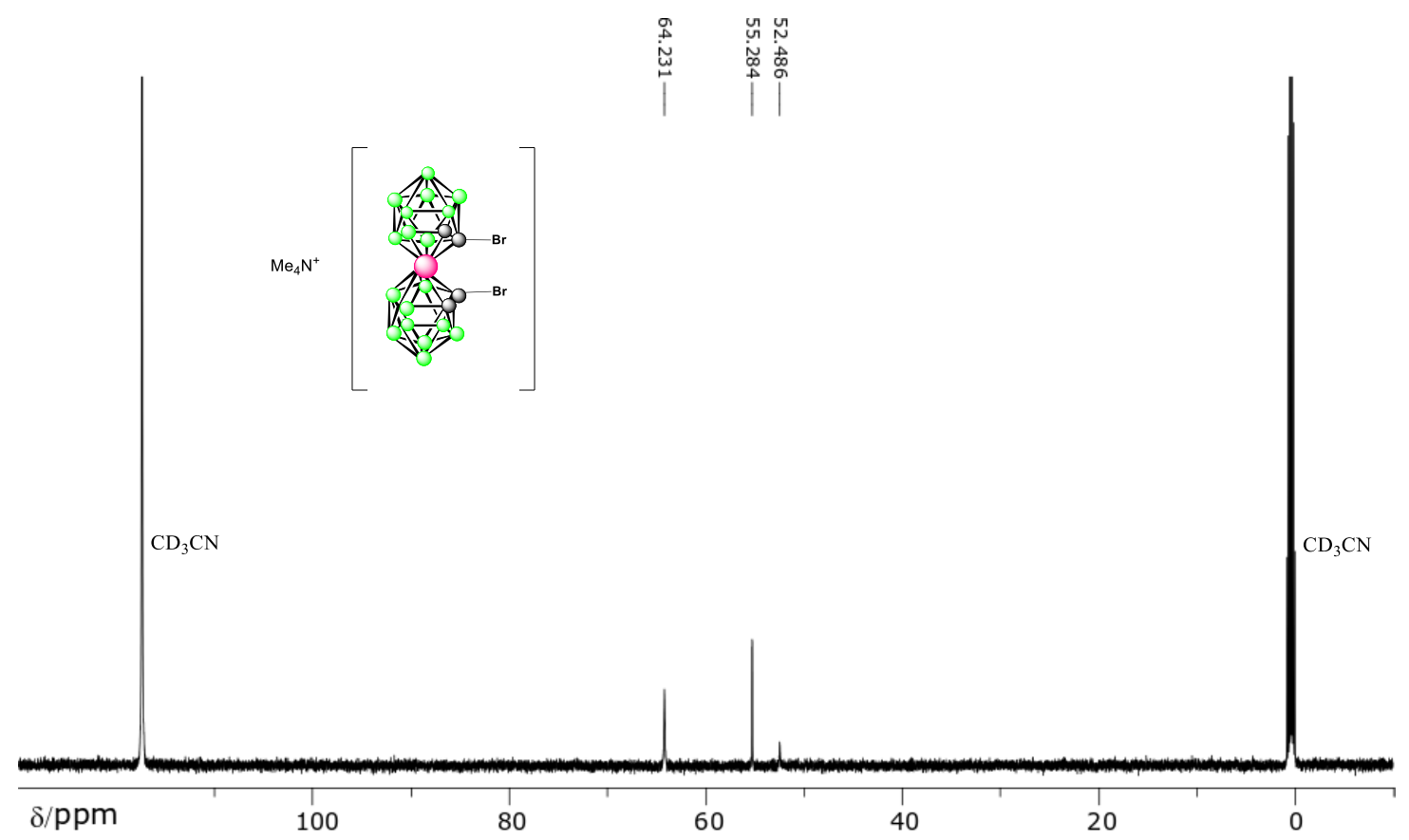

Figure S14. NMR spectra of crude compound $\mathbf{6}^{-}$(diastereoisomers) and $\mathrm{rac}^{-6} \mathbf{6}^{-}$in $\mathrm{CD}_{3} \mathrm{CN}$, from top to bottom: a) ${ }^{11} \mathrm{~B}\left\{{ }^{1} \mathrm{H}\right\} \mathrm{NMR}$ $(192 \mathrm{MHz})$, b) ${ }^{1} \mathrm{H}$ NMR $\left.(600 \mathrm{MHz}), \mathrm{c}\right){ }^{13} \mathrm{C}$ NMR $(150 \mathrm{MHz})$; d ${ }^{11} \mathrm{~B}\left\{{ }^{1} \mathrm{H}\right\}$ NMR for $\mathrm{rac}^{-6} \mathbf{6}^{-}(192 \mathrm{MHz})$, e) ${ }^{1} \mathrm{H}$ NMR for $\mathrm{rac}^{-6^{-}}$ $(600 \mathrm{MHz}), \mathrm{f}){ }^{13} \mathrm{C}$ NMR for rac-6 $\mathbf{6}^{-}(150 \mathrm{MHz})$ 
3. NMR spectra for compounds containing tetrazole rings by dipolar cycloadditions

a)
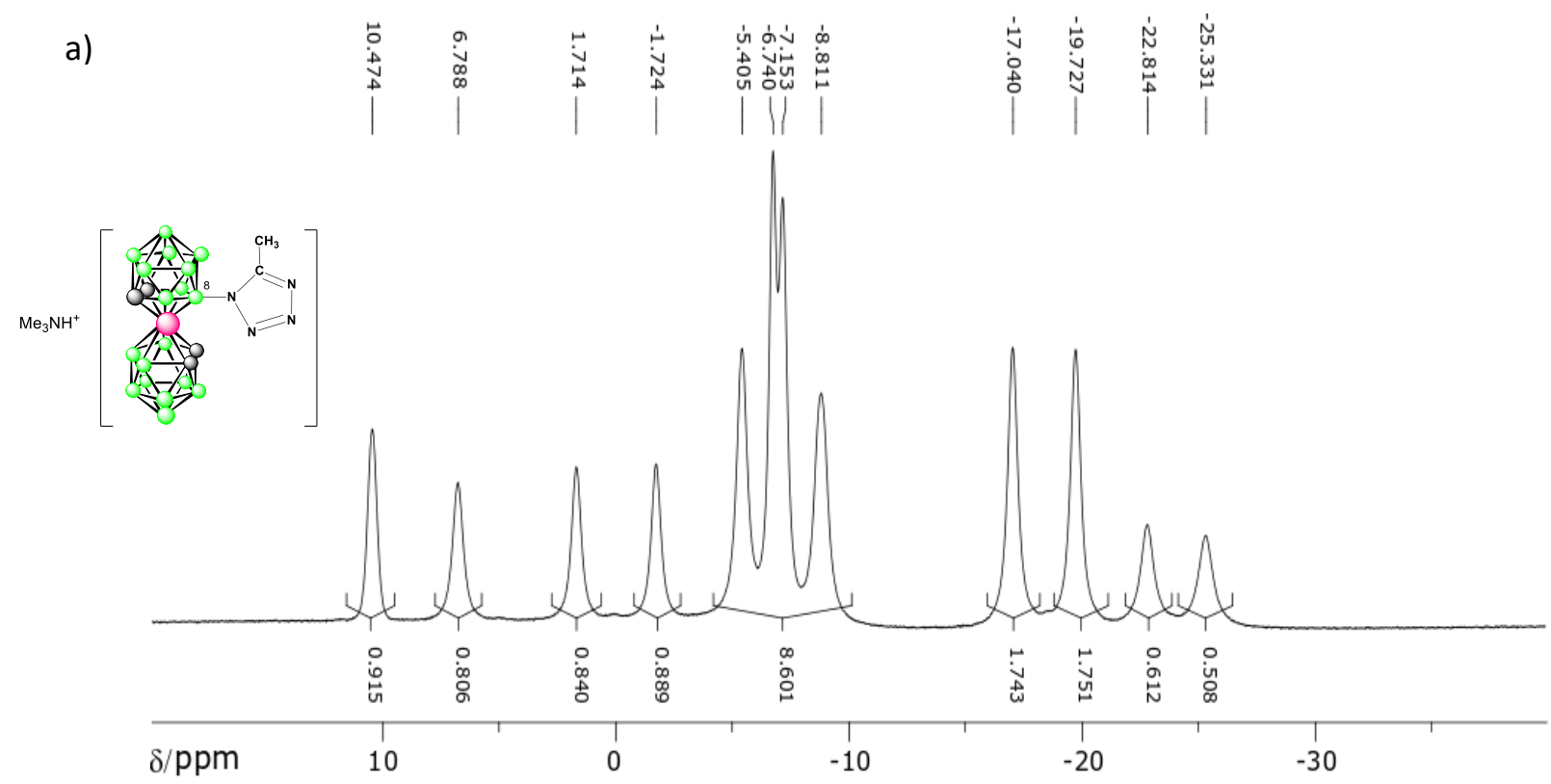

b)

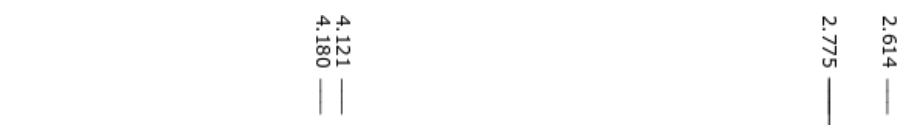

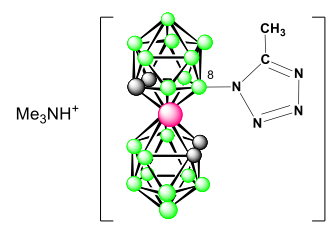

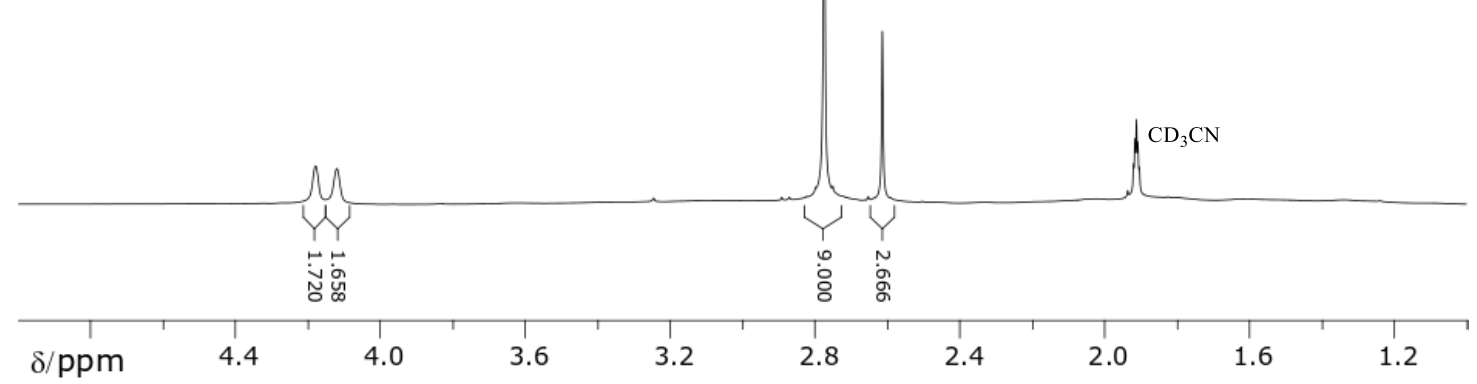


c)

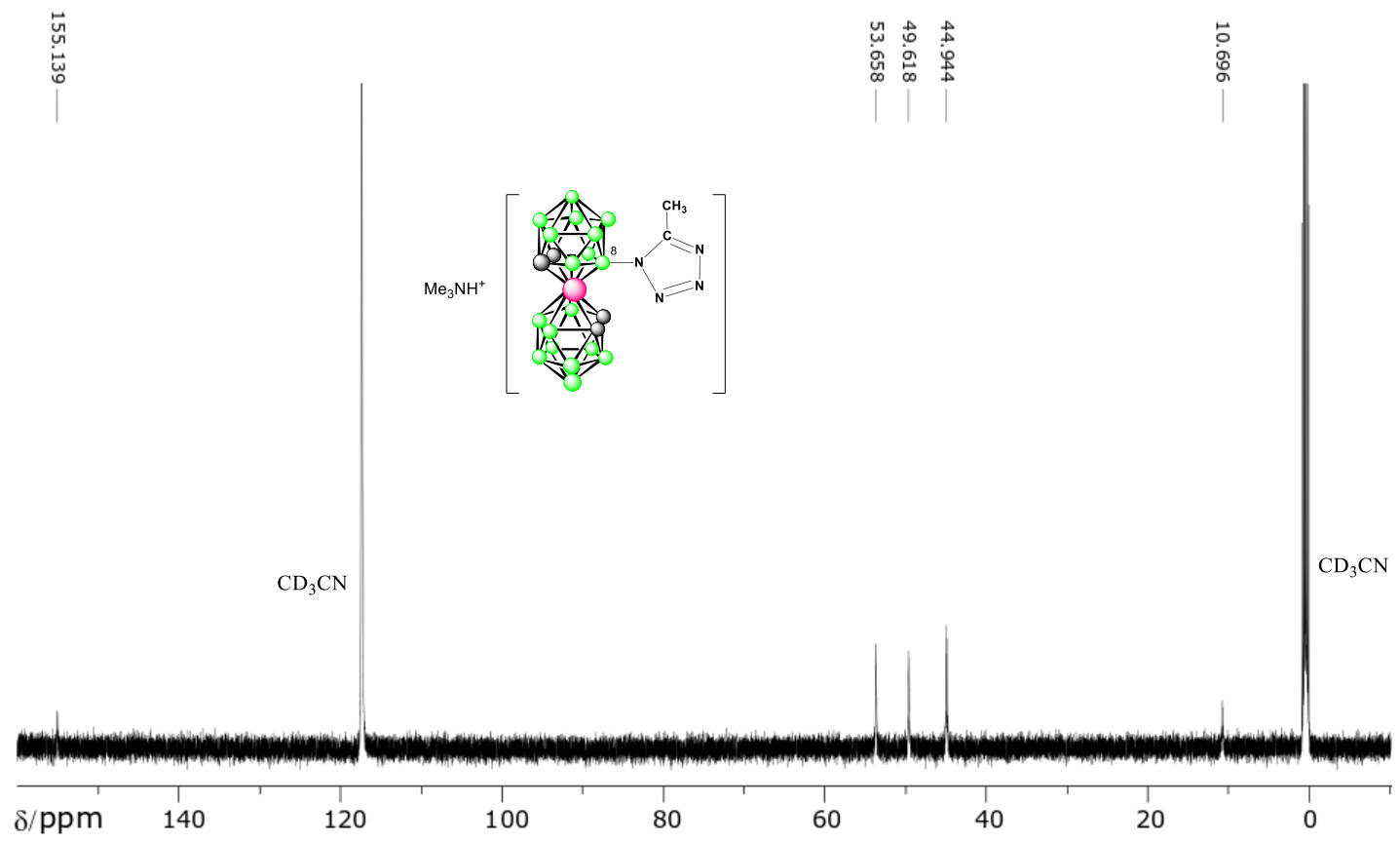

Figure S15. NMR spectra of compound $7^{-}$in $\mathrm{CD}_{3} \mathrm{CN}$, from top to bottom: a) $\left.{ }^{11} \mathrm{~B}\left\{{ }^{1} \mathrm{H}\right\} \mathrm{NMR}(192 \mathrm{MHz}), \mathrm{b}\right){ }^{1} \mathrm{H} \mathrm{NMR}(600 \mathrm{MHz})$, c) ${ }^{13} \mathrm{C}$ NMR $(150 \mathrm{MHz})$

a)

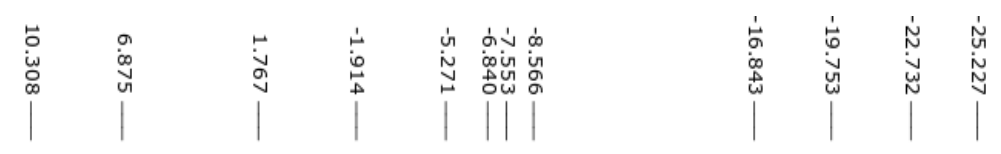

$\mathrm{Me}_{3} \mathrm{NH}^{+}$

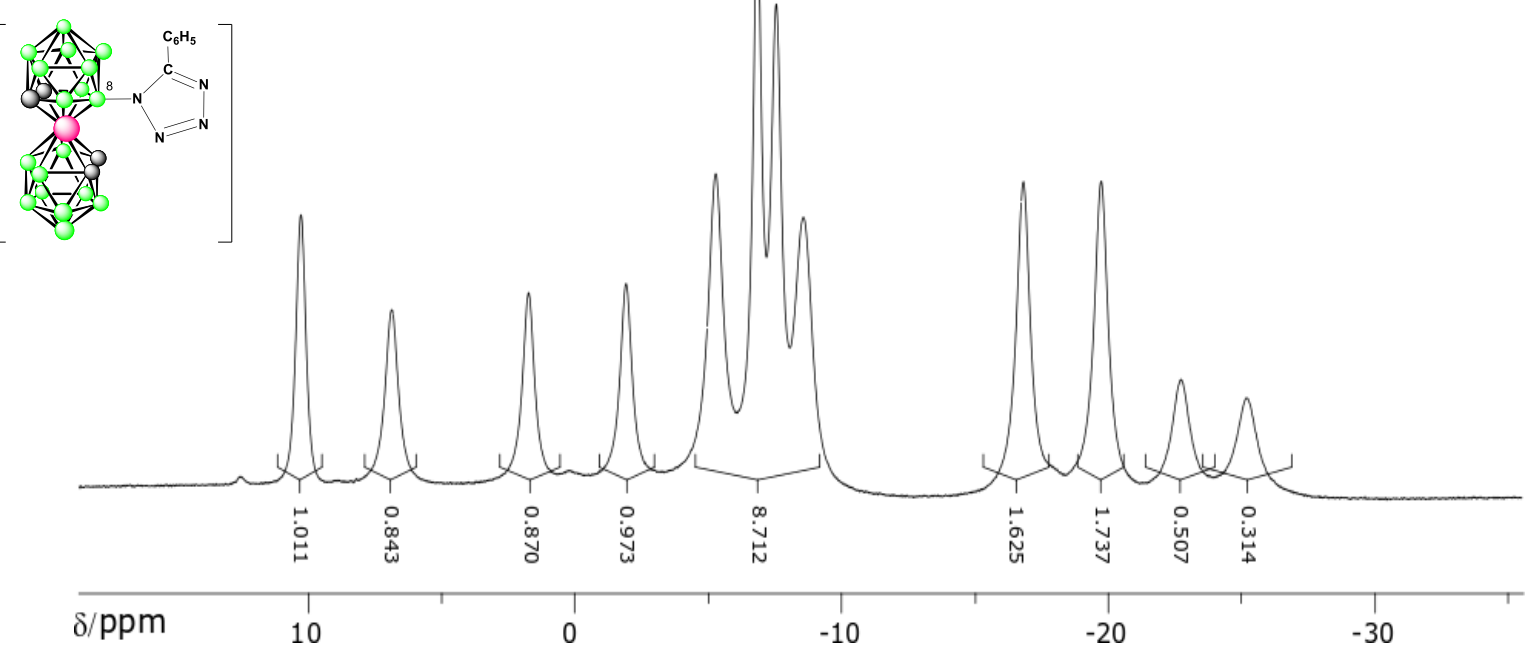


b)

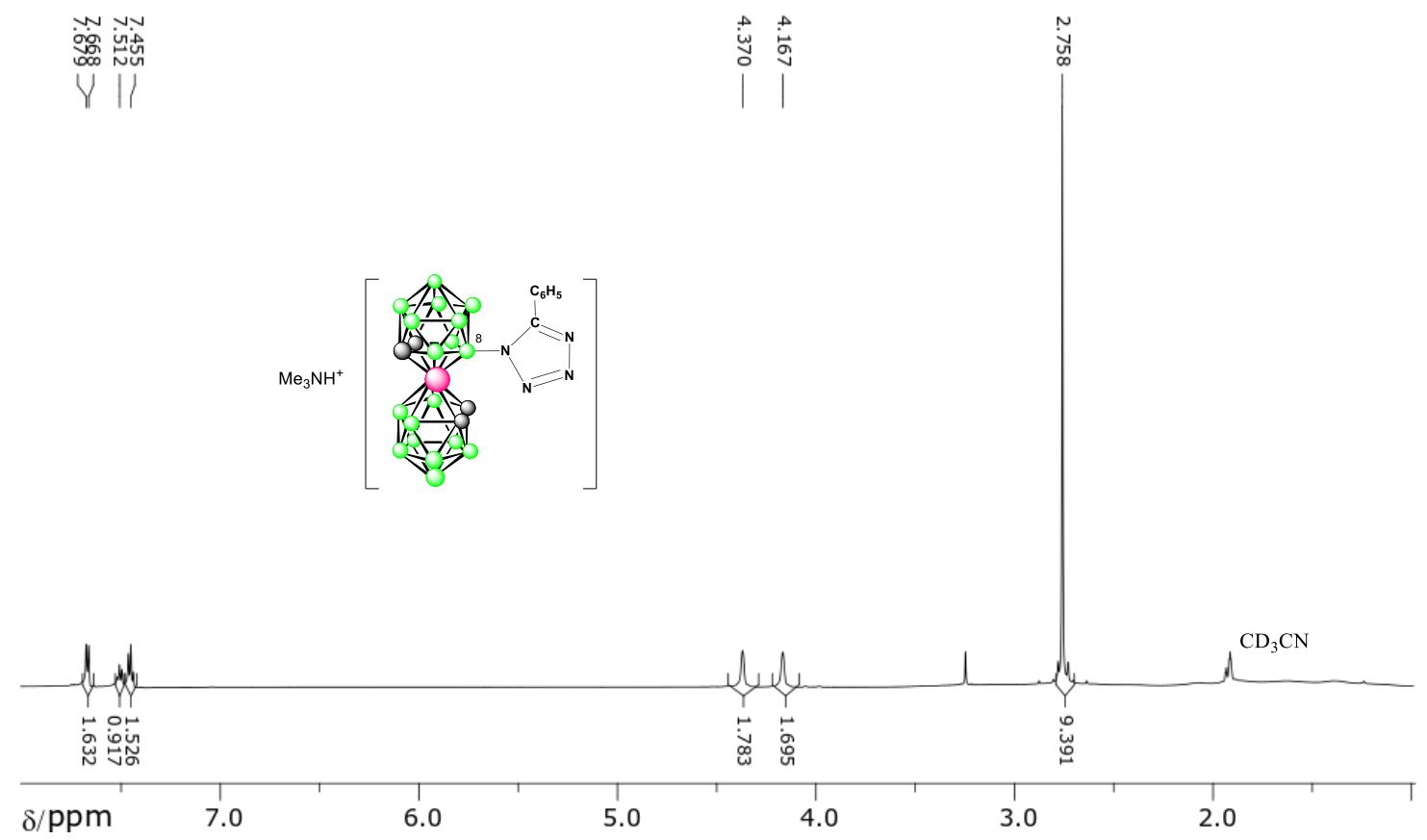

c)

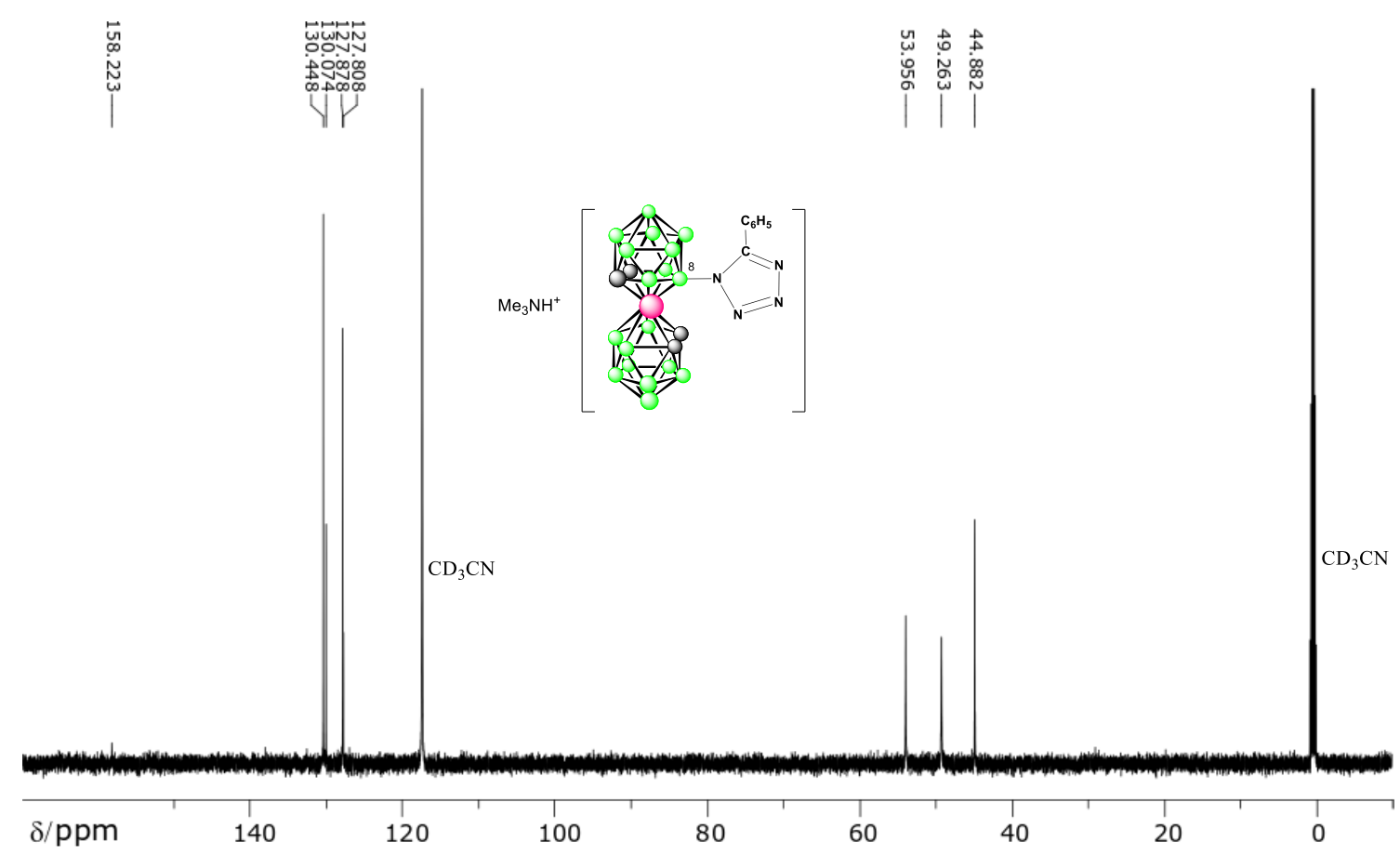

Figure S16. NMR spectra of compound $8^{-}$in $\mathrm{CD}_{3} \mathrm{CN}$, from top to bottom: a) ${ }^{11} \mathrm{~B}\left\{{ }^{1} \mathrm{H}\right\} \mathrm{NMR}(192 \mathrm{MHz})$, b) ${ }^{1} \mathrm{H}$ NMR $(600 \mathrm{MHz})$, c) ${ }^{13} \mathrm{C}$ NMR $(150 \mathrm{MHz})$ 
a)

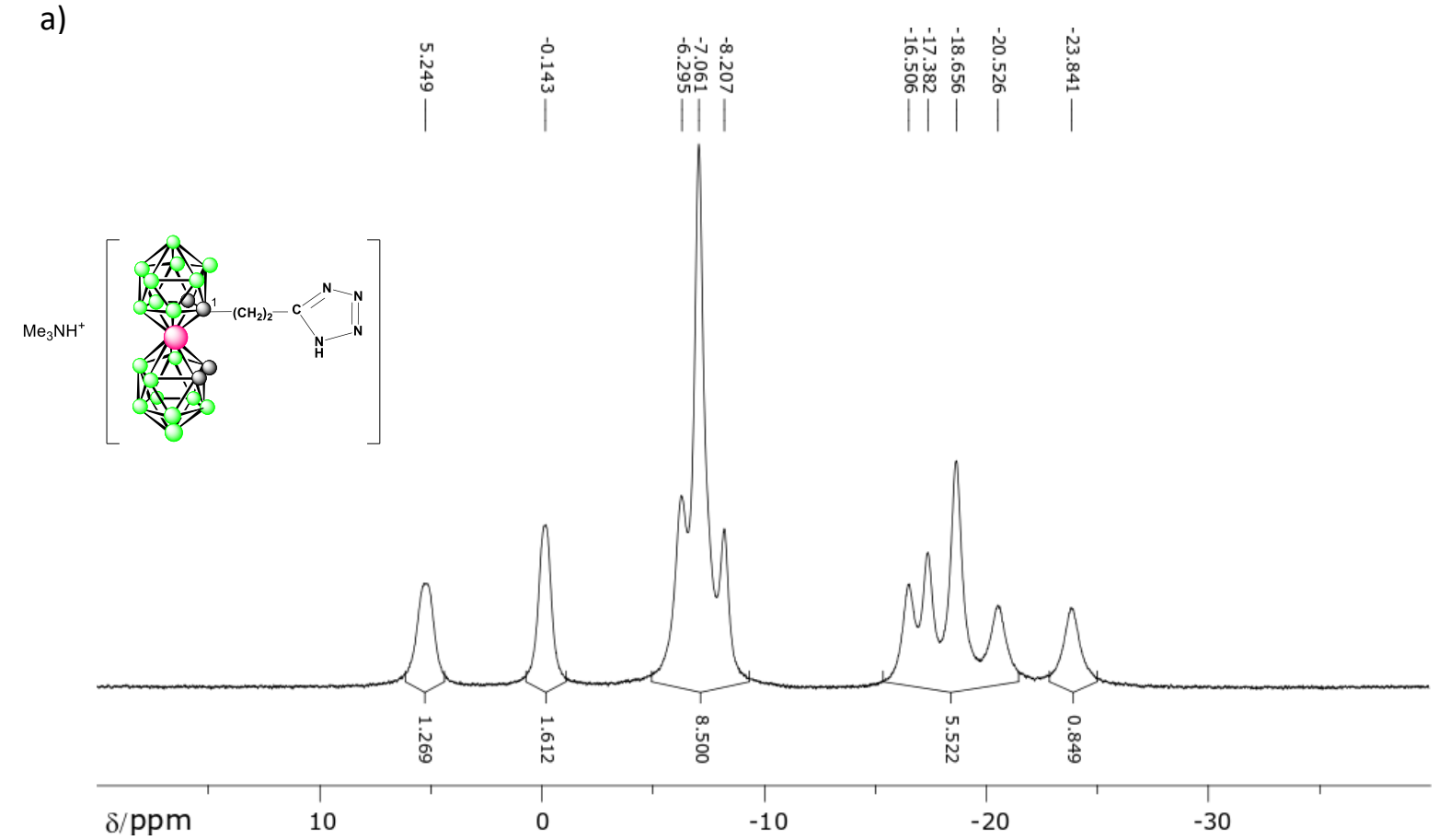

b)

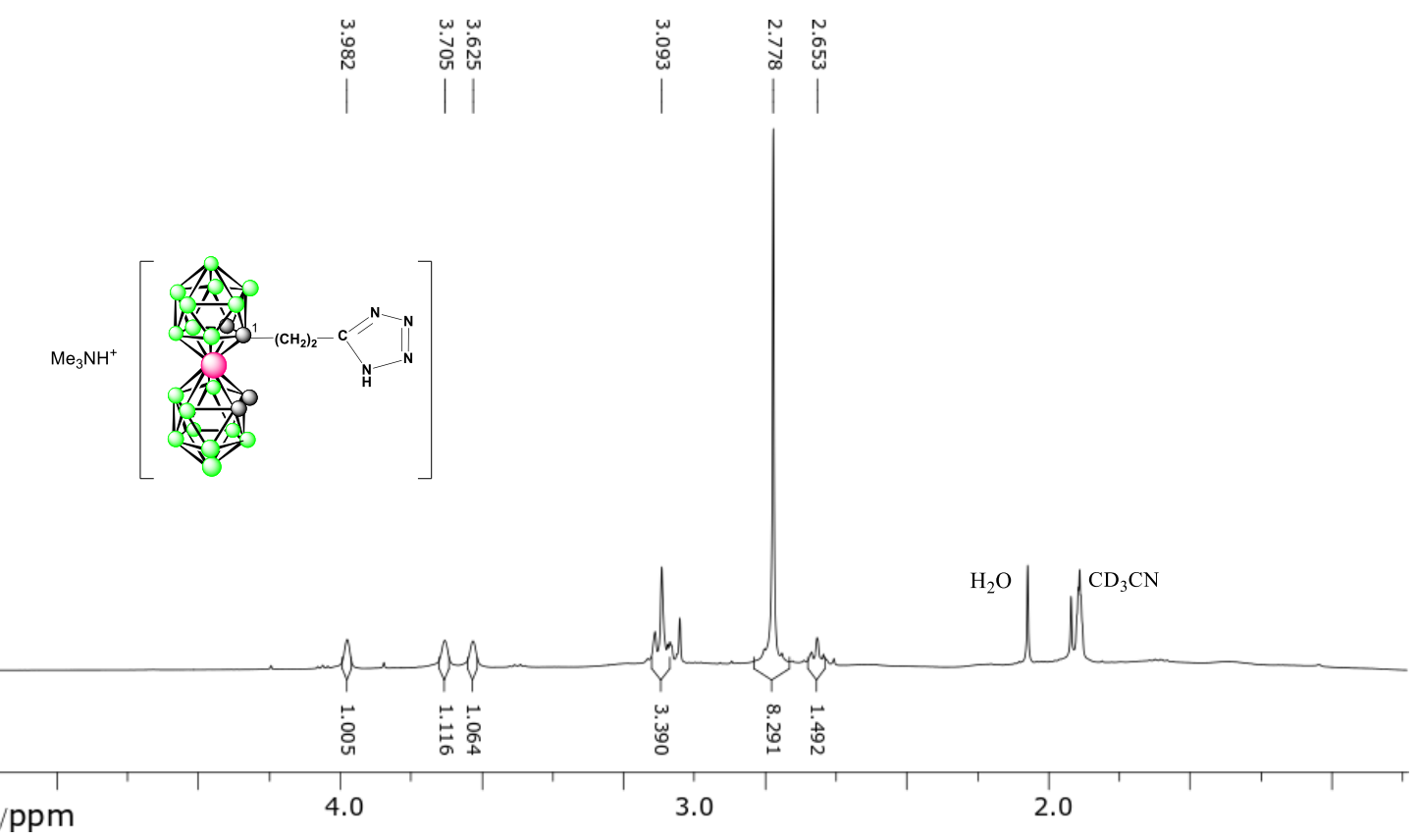


c)

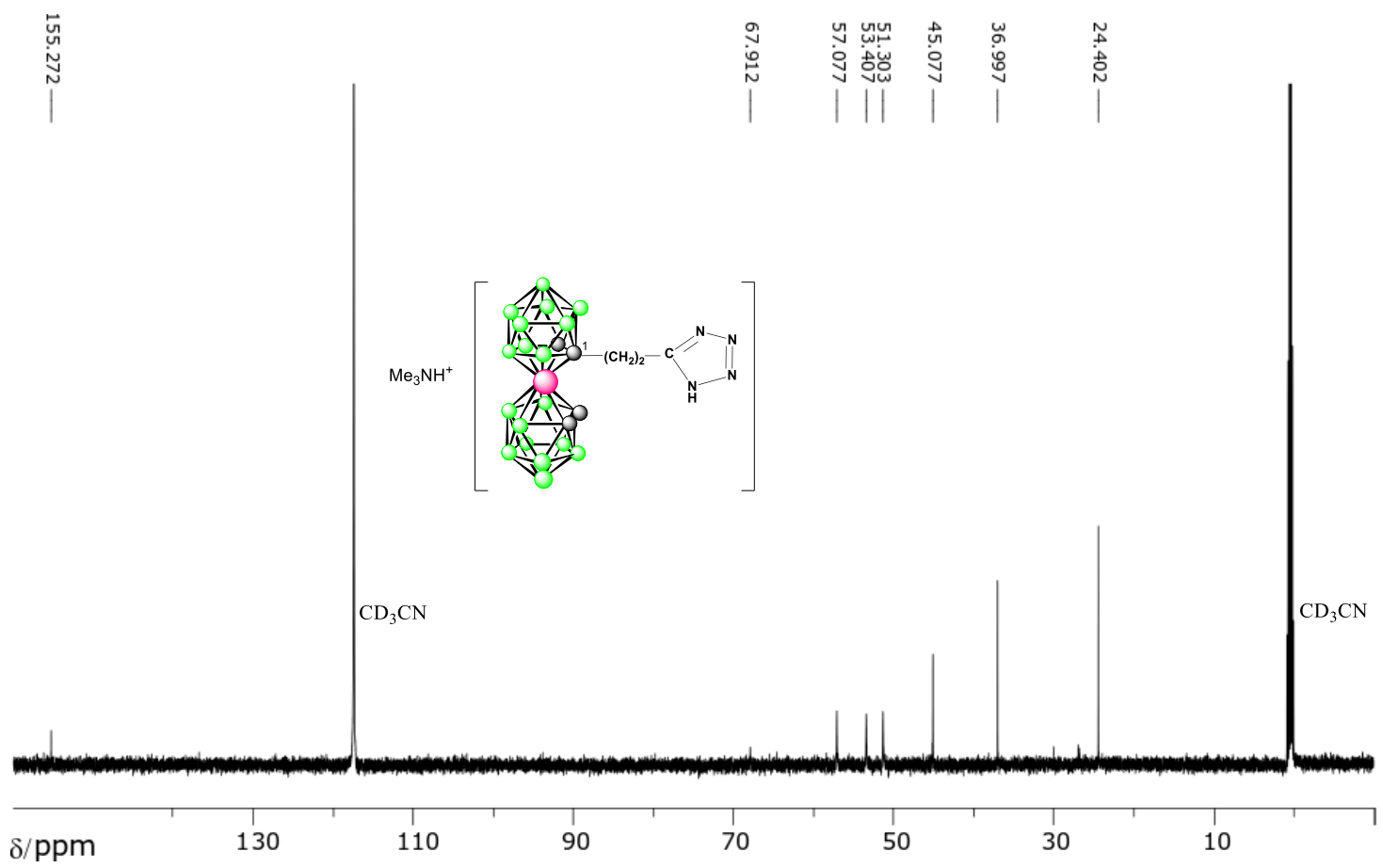

Figure S17. NMR spectra of compound $9^{-}$in $\mathrm{CD}_{3} \mathrm{CN}$, from top to bottom: a) ${ }^{11} \mathrm{~B}\left\{{ }^{1} \mathrm{H}\right\} \mathrm{NMR}(192 \mathrm{MHz})$, b) ${ }^{1} \mathrm{H}$ NMR (600 MHz), c) ${ }^{13} \mathrm{C}$ NMR $(150 \mathrm{MHz})$

a)
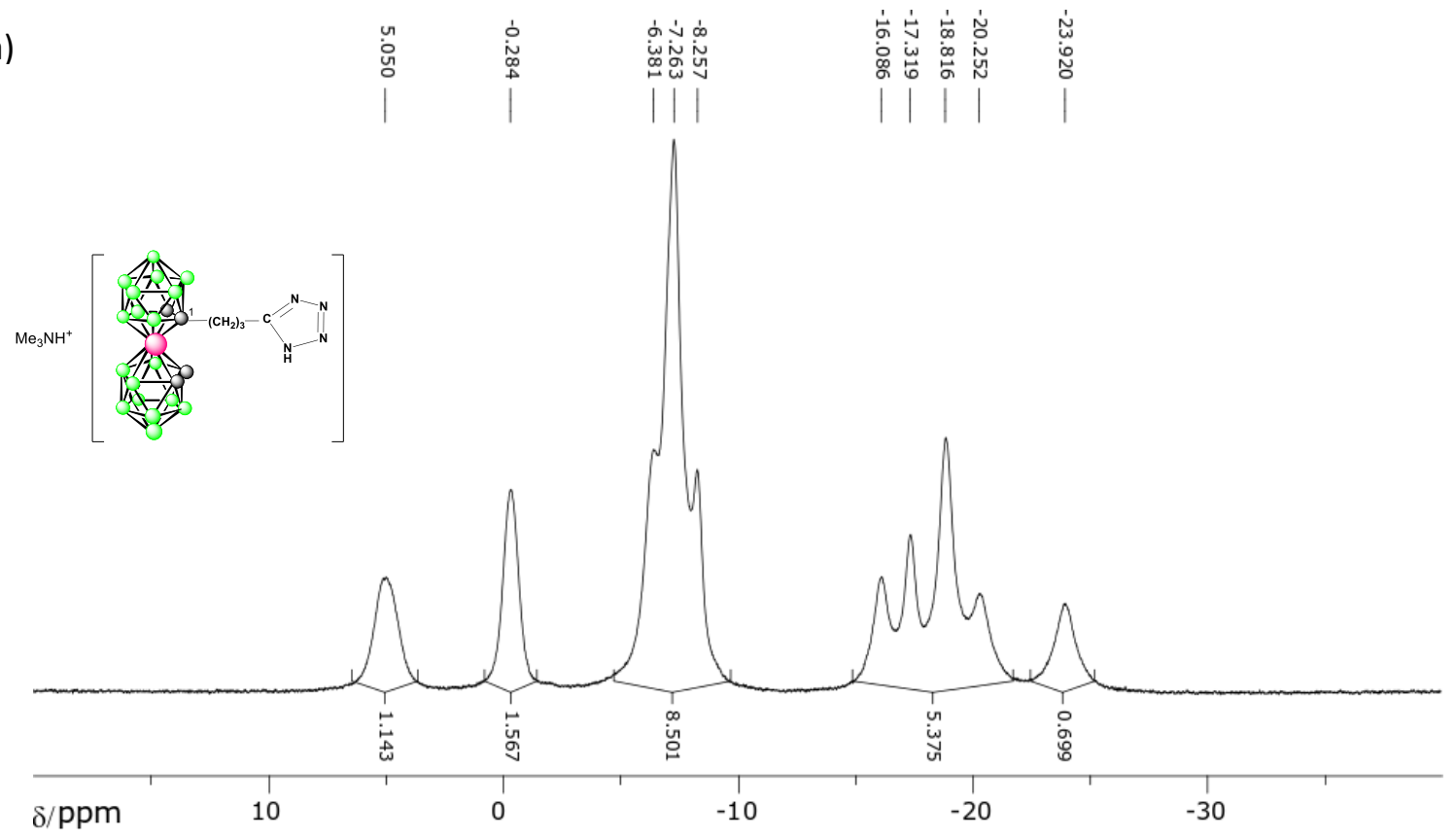


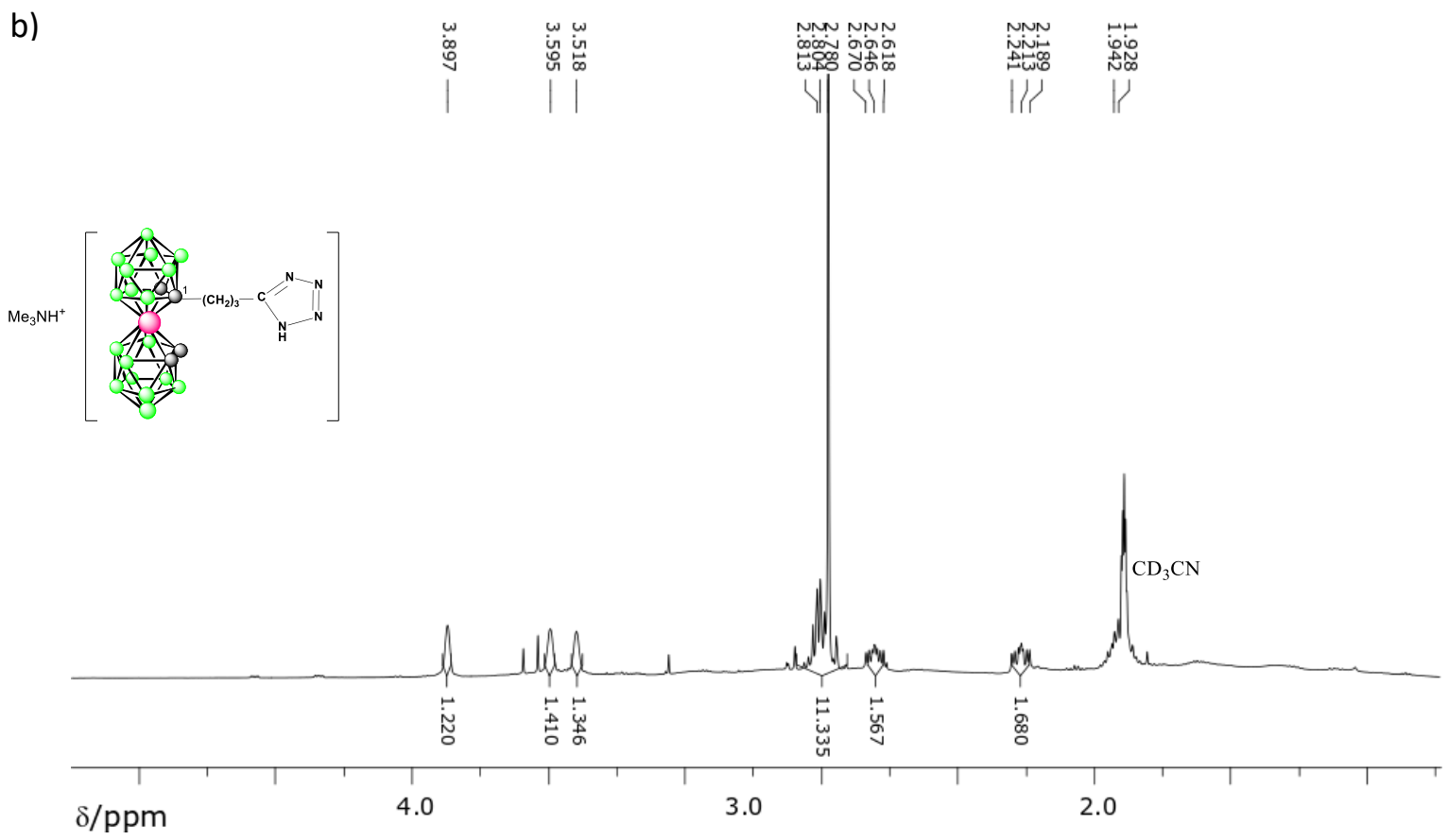

c)

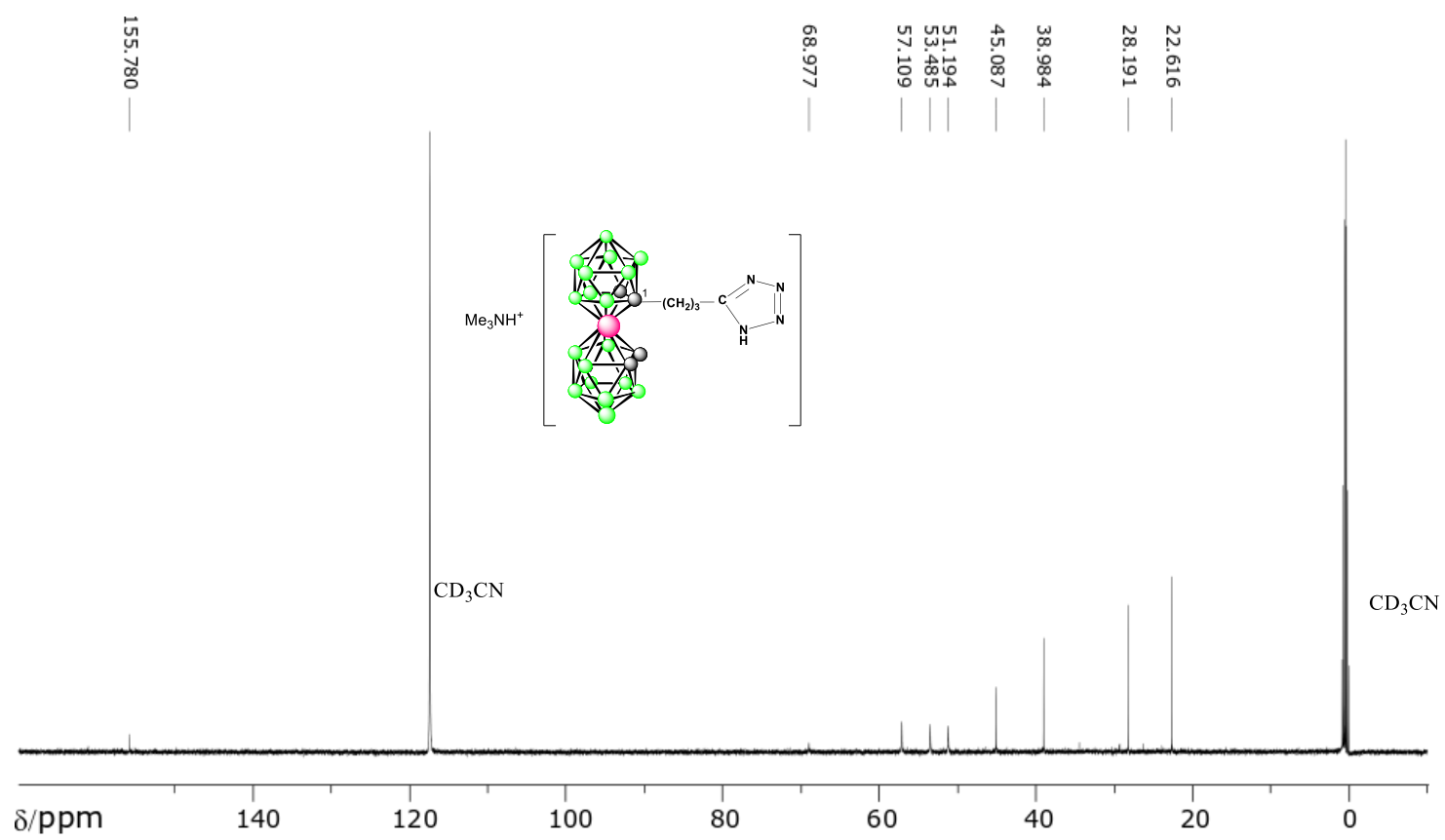

Figure S18. NMR spectra of compound $10^{-}$in $\mathrm{CD}_{3} \mathrm{CN}$, from top to bottom: a) ${ }^{11} \mathrm{~B}\left\{{ }^{1} \mathrm{H}\right\}$ NMR (192 MHz), b) ${ }^{1} \mathrm{H}$ NMR (600 $\mathrm{MHz}), \mathrm{c}){ }^{13} \mathrm{C}$ NMR (150 MHz) 
a)
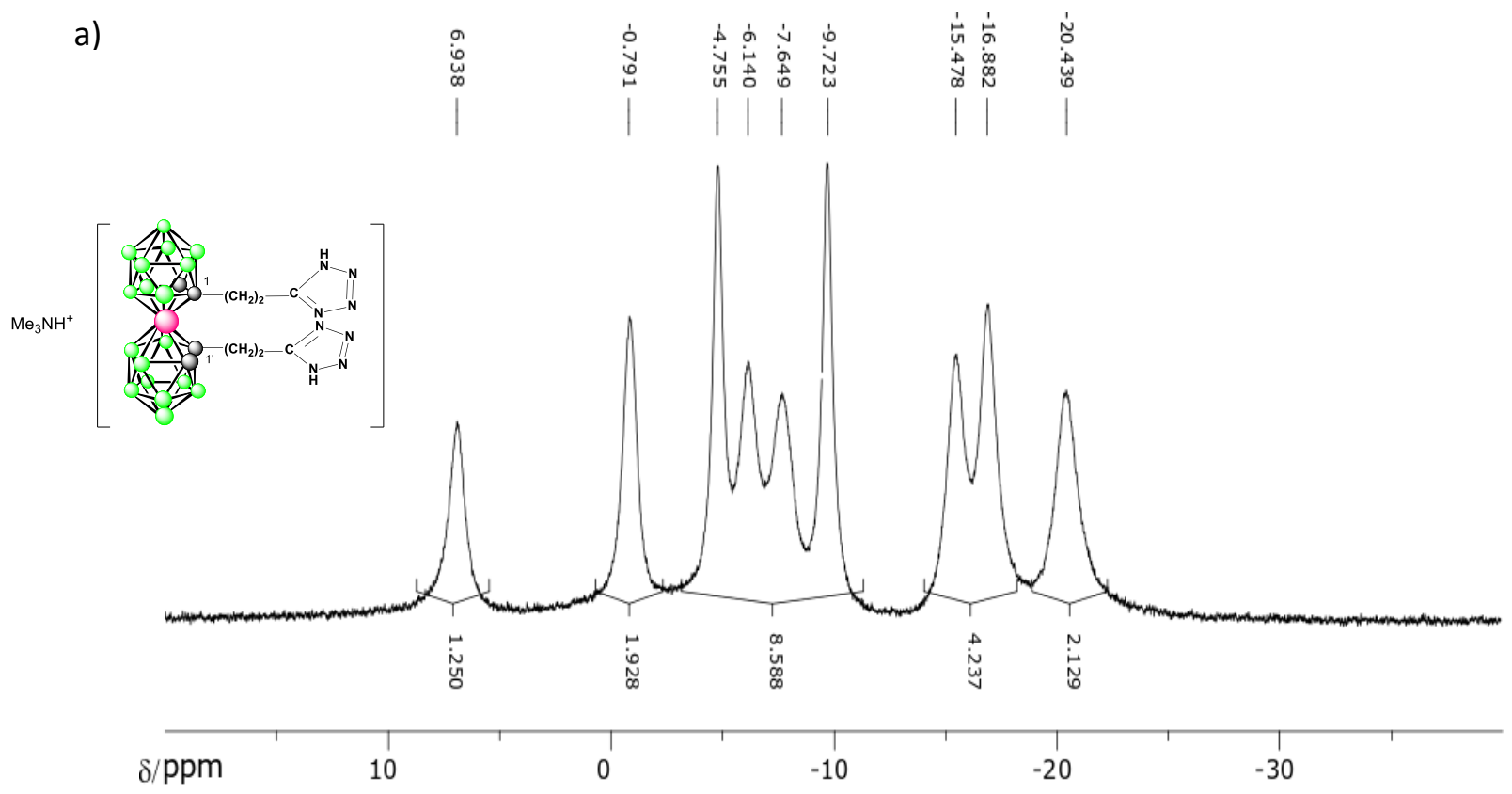

b)
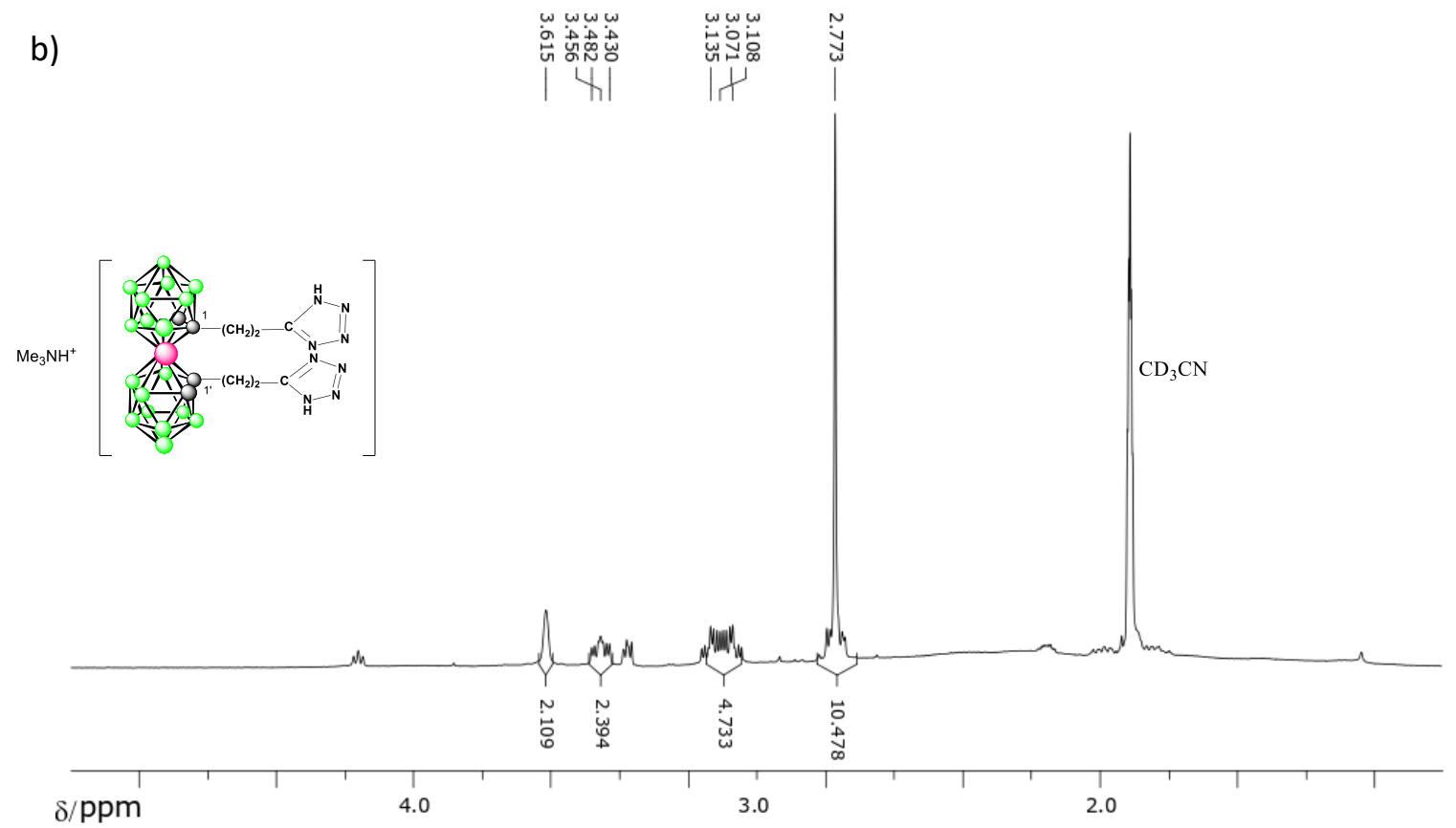


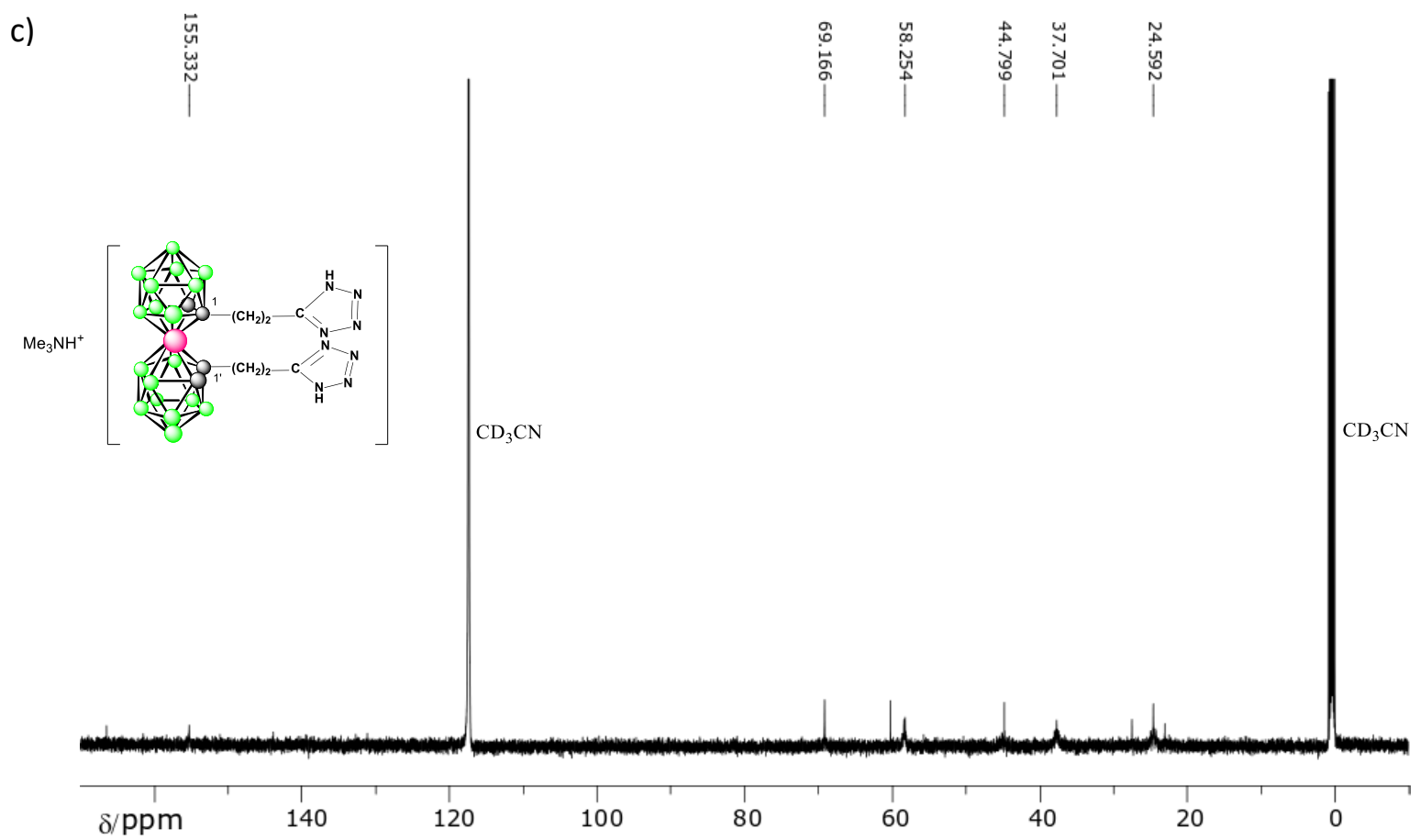

Figure S19. NMR spectra of compound $\mathbf{1 1}^{-}$in $\mathrm{CD}_{3} \mathrm{CN}$, from top to bottom: a) $\left.{ }^{11} \mathrm{~B}\left\{{ }^{1} \mathrm{H}\right\} \mathrm{NMR}(192 \mathrm{MHz}), \mathrm{b}\right){ }^{1} \mathrm{H} \mathrm{NMR}(600$ $\mathrm{MHz}), \mathrm{c}){ }^{13} \mathrm{C}$ NMR $(150 \mathrm{MHz})$

a)

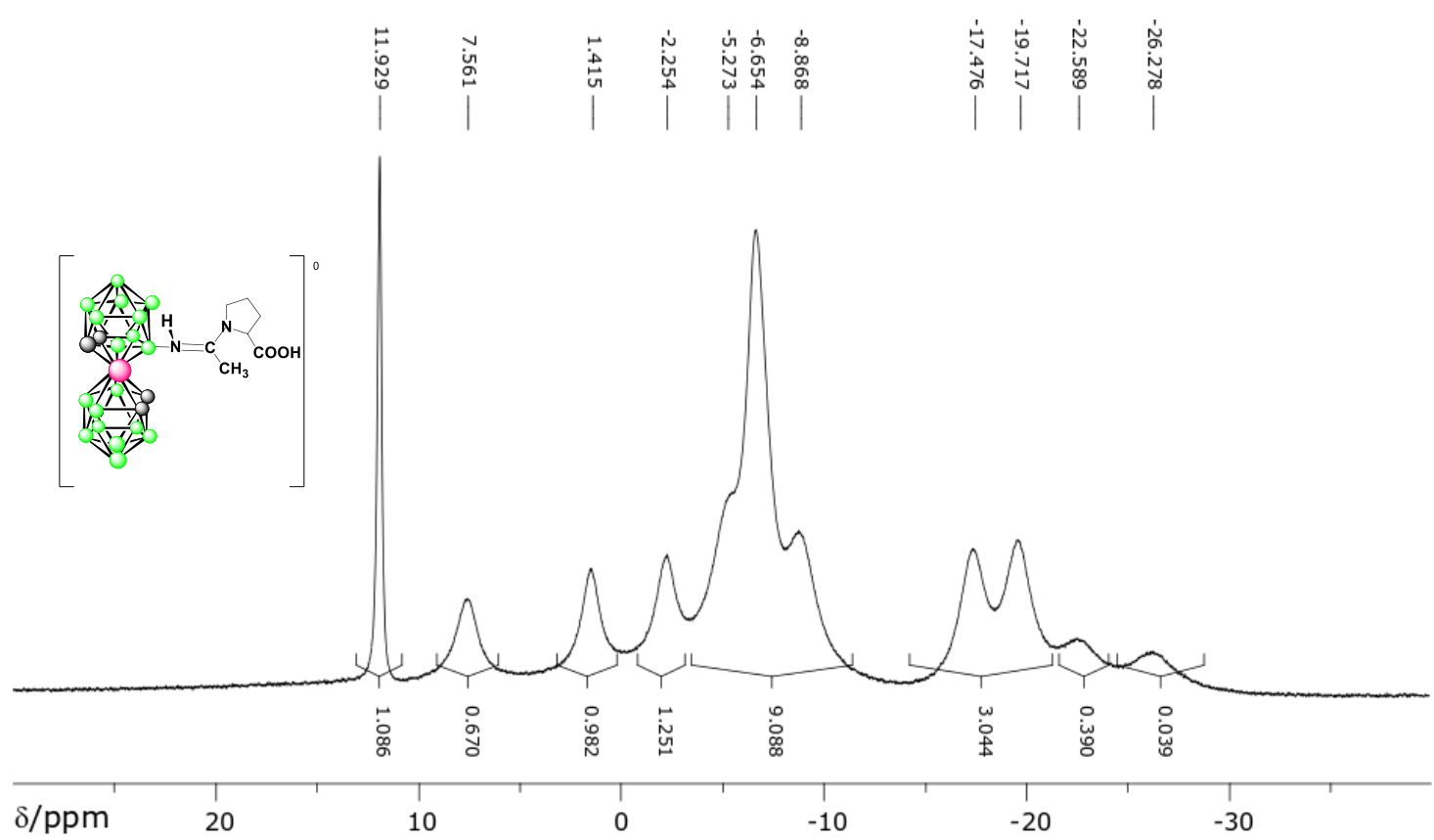


b)
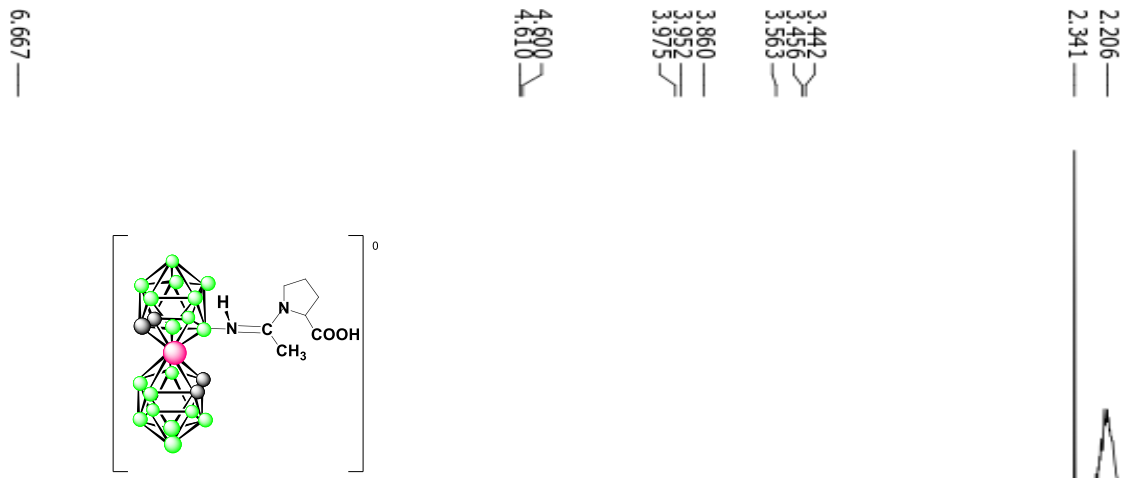

c)

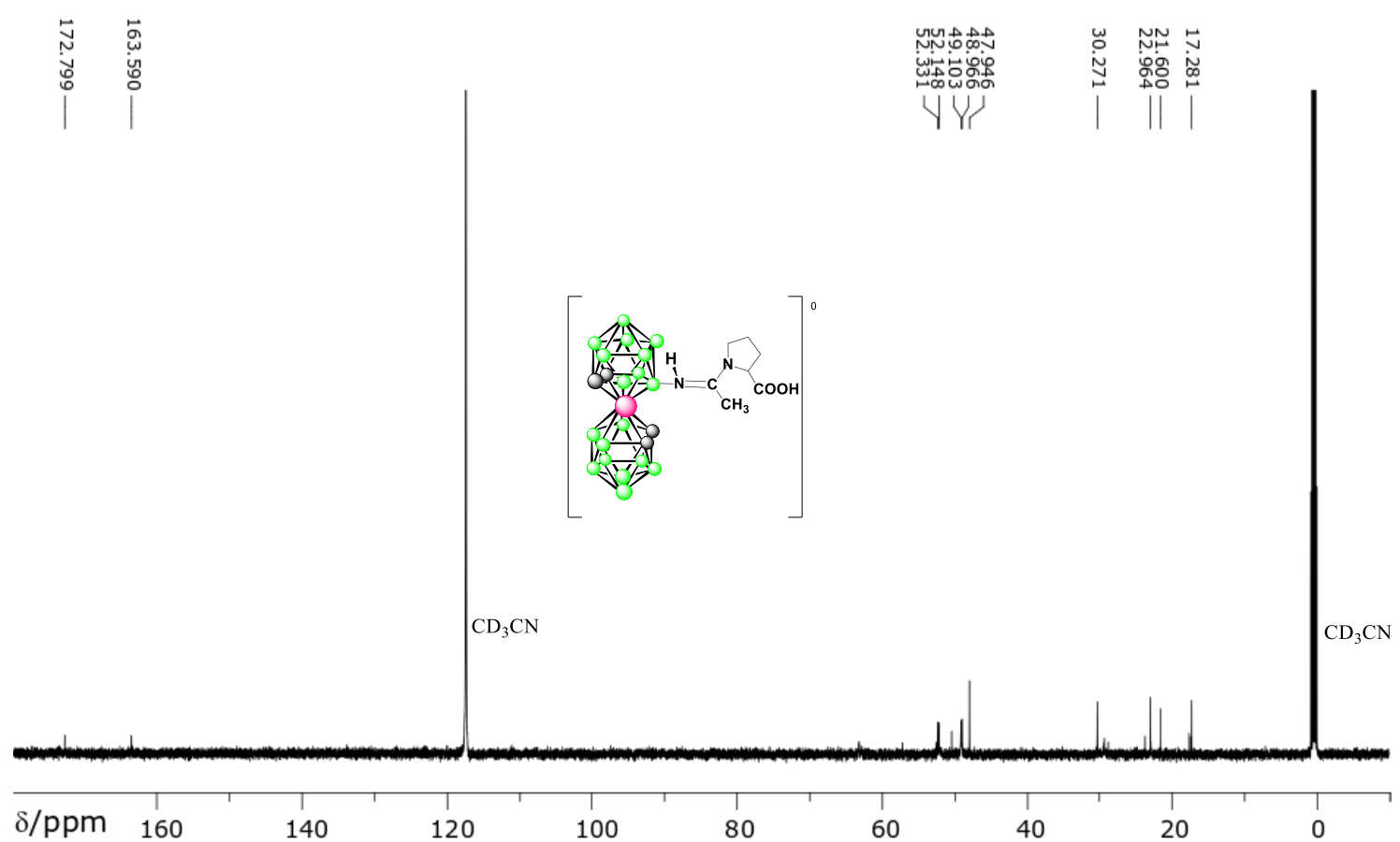

Figure S20. NMR spectra of compound 12 in $\mathrm{CD}_{3} \mathrm{CN}$, from top to bottom: a) ${ }^{11} \mathrm{~B}\left\{{ }^{1} \mathrm{H}\right\}$ NMR (192 MHz), b) ${ }^{1} \mathrm{H}$ NMR (600 $\mathrm{MHz}), \mathrm{c}){ }^{13} \mathrm{C}$ NMR $(150 \mathrm{MHz})$ 


\section{Mass Spectra}

1. MS spectra for nitrilium derivatives of cobalt bis(dicarbollide) ions

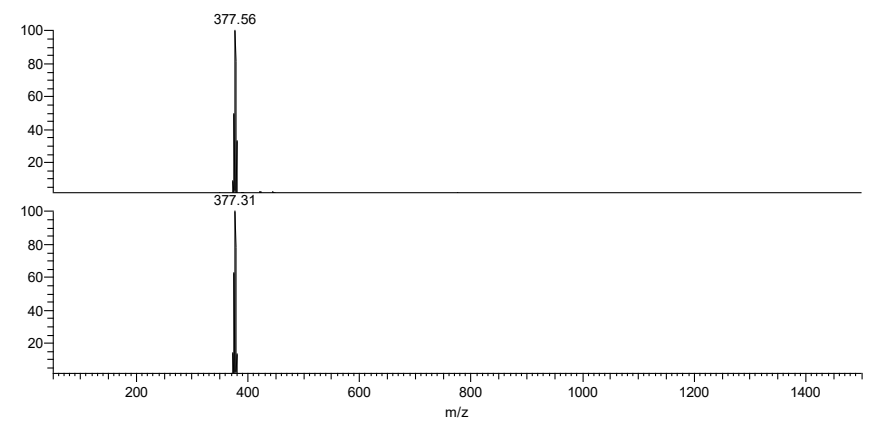

$$
\begin{array}{ll}
\text { NL: } \\
1.76 E 4
\end{array}
$$

Compound 2.\#1 RT: 0.00 AV: 1 T: ITMS - P ESIE
Full ms $[50.00-1500.00]$

NL:
$5.37 E 3$

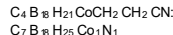

$\mathrm{p}(\mathrm{gss}, \mathrm{s} / \mathrm{p}: 40)$ Chrg
R: 0.4 Da @FWHM

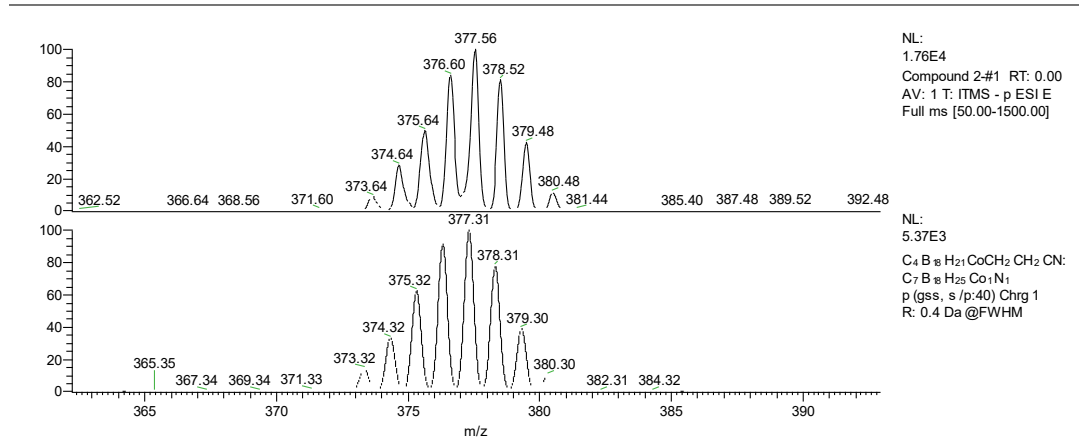

Figure S21. MS of compound $\mathbf{2}^{-}$
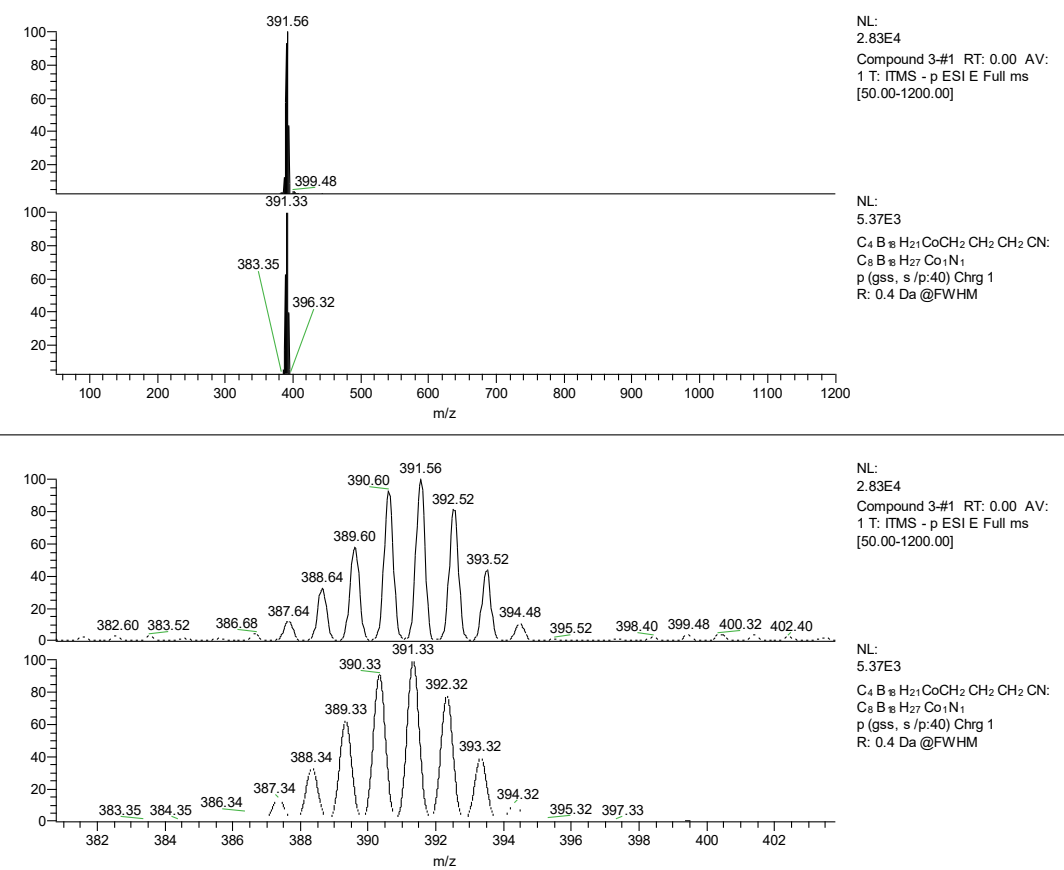

Figure S22. MS of compound $\mathbf{3}^{-}$ 

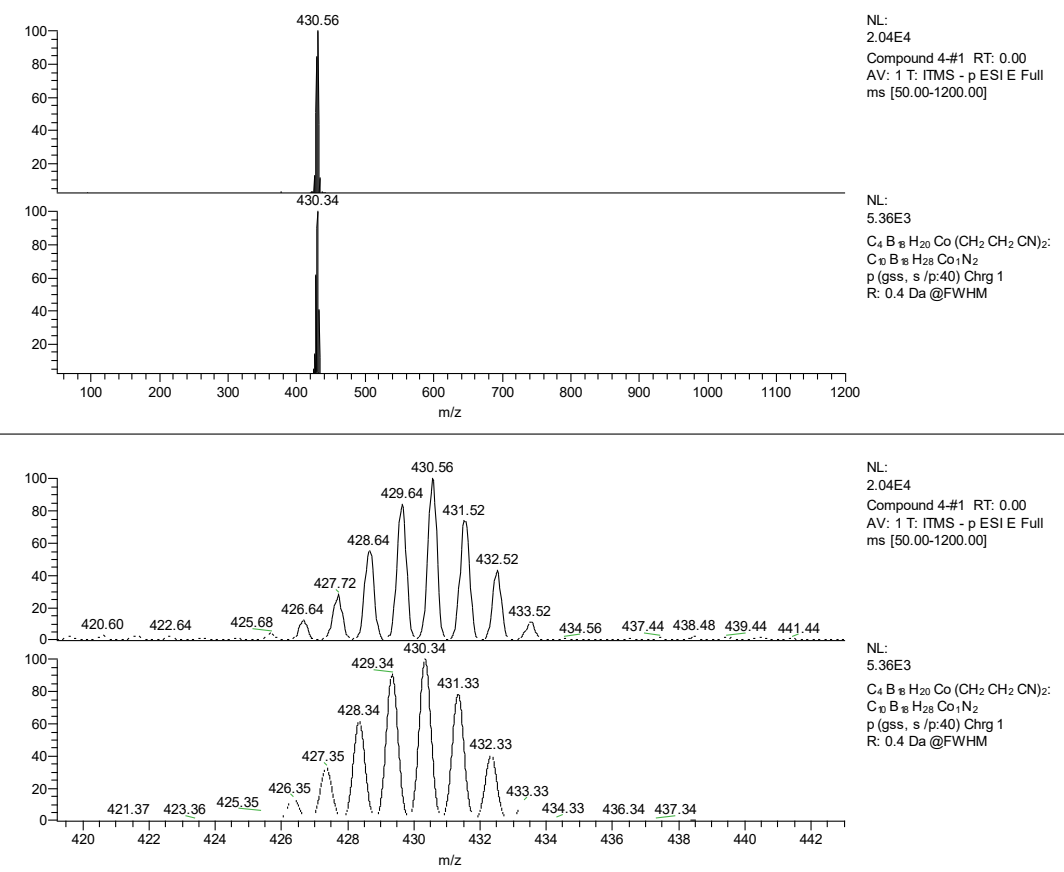

Figure S23. MS of compound $\mathbf{4}^{-}$

2. MS spectra for brominated derivatives of cobalt bis(dicarbollide) ions
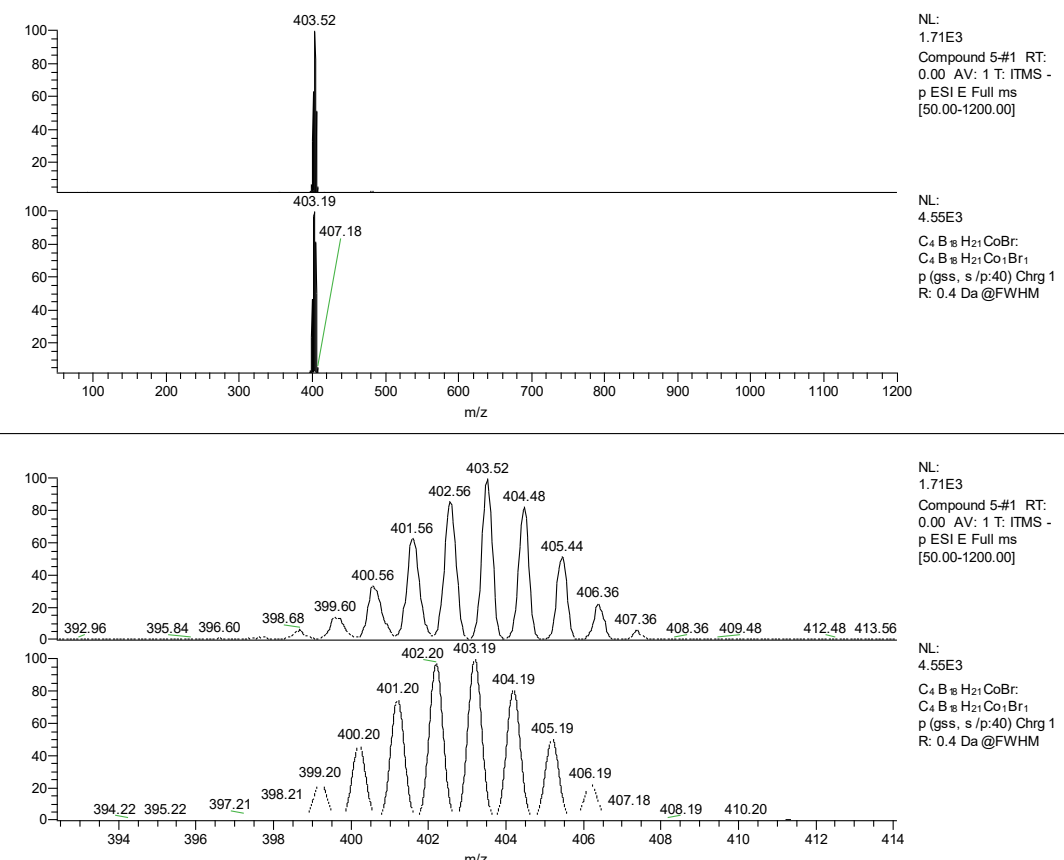

Figure S24. MS of compound 5 


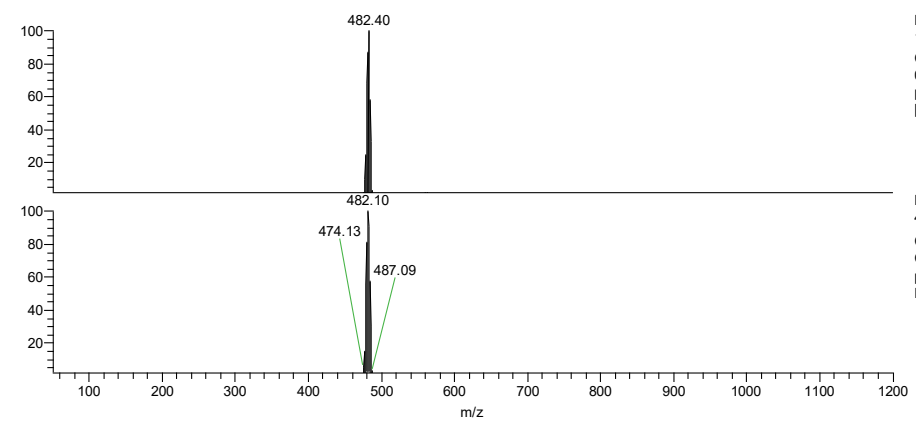

NL:
$1.99 E 4$

Compound 6-\#1 RT:
0.00 AV: 1 T: ITMS

$\mathrm{p}$ ESIE Full ms
$[50.00-1200.00]$

NL:

$\mathrm{C}_{4} \mathrm{~B}_{3 \mathrm{~B}} \mathrm{H}_{20} \mathrm{COBBr}_{2}:$

p (gss, s/p:40) Chrg 1

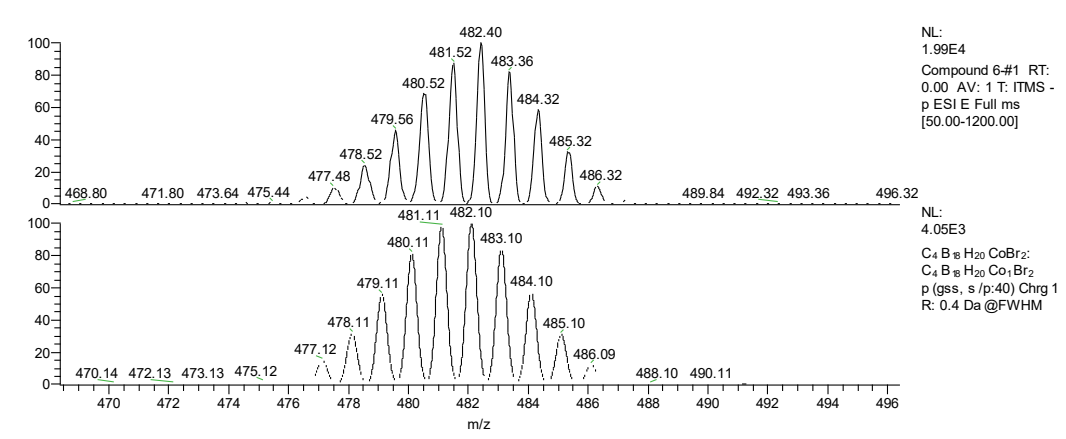

Figure S25. MS of compound $6^{-}$

3. MS spectra for compounds containing tetrazole rings by dipolar cycloadditions
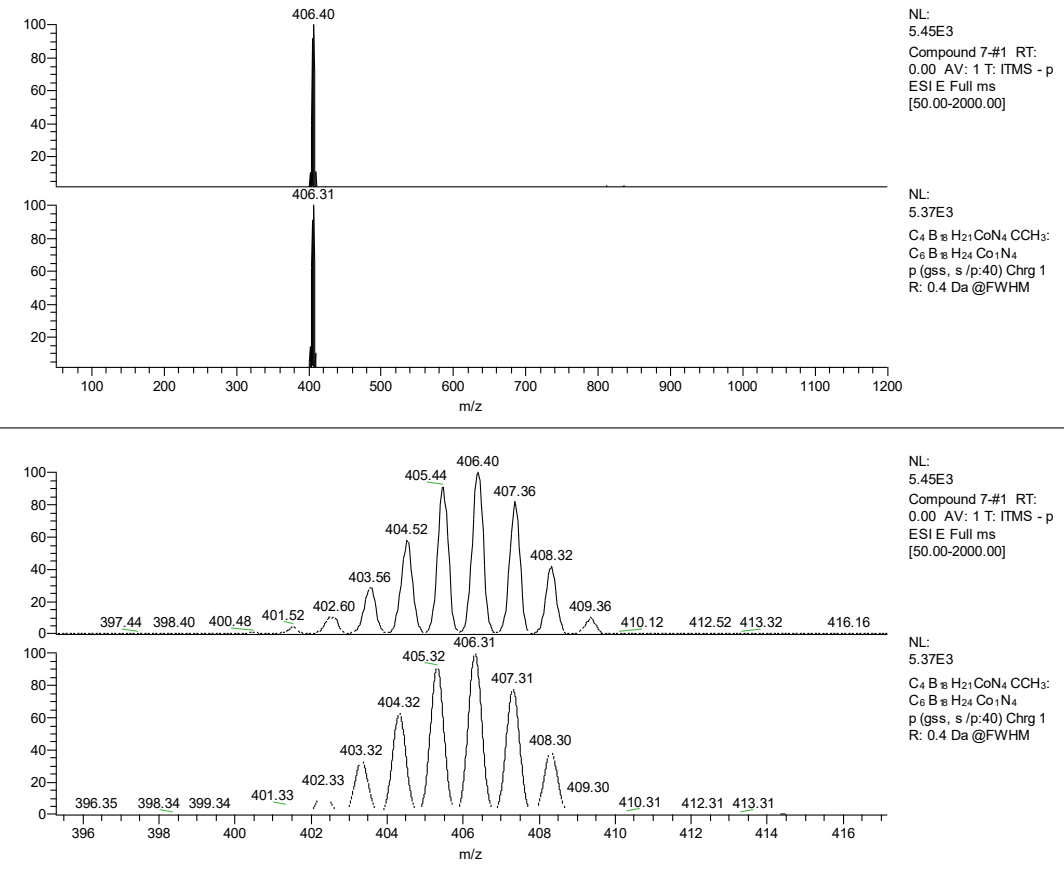

Figure S26. MS of compound $7^{-}$ 


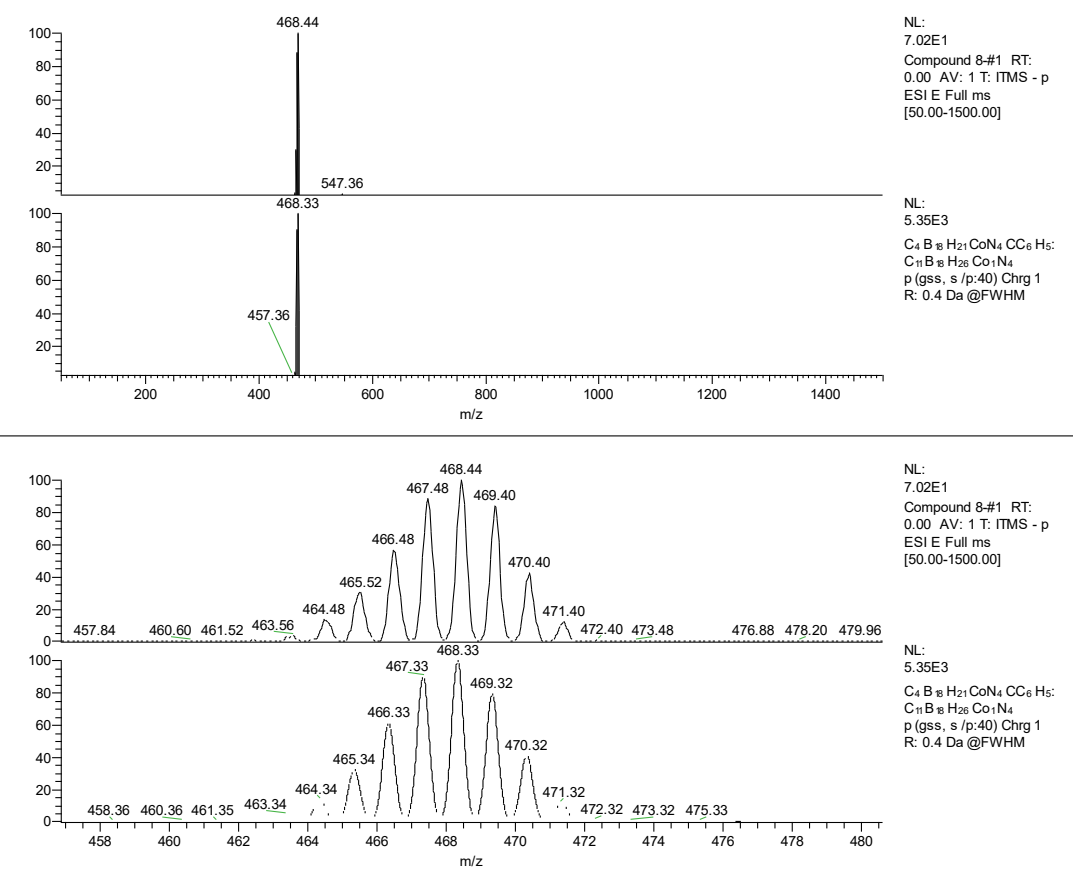

Figure S27. MS of compound $\mathbf{8}^{-}$

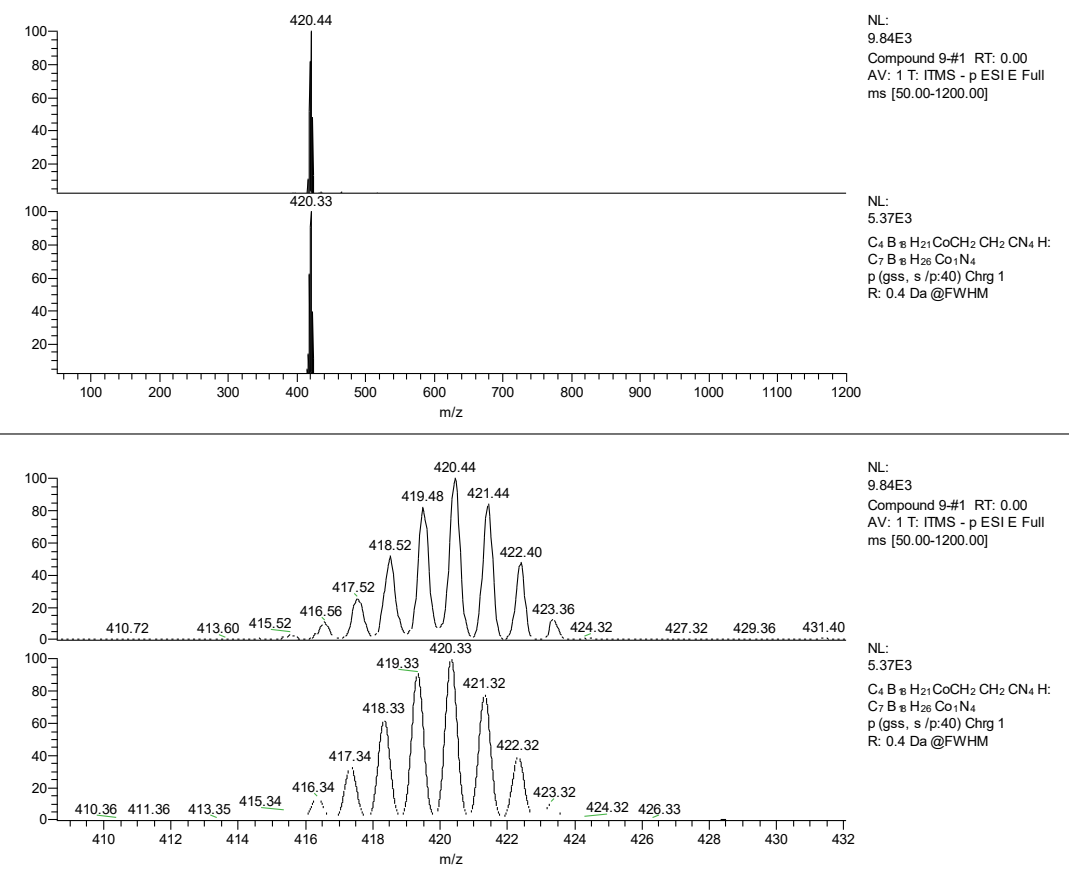

Figure S28. MS of compound 9- 

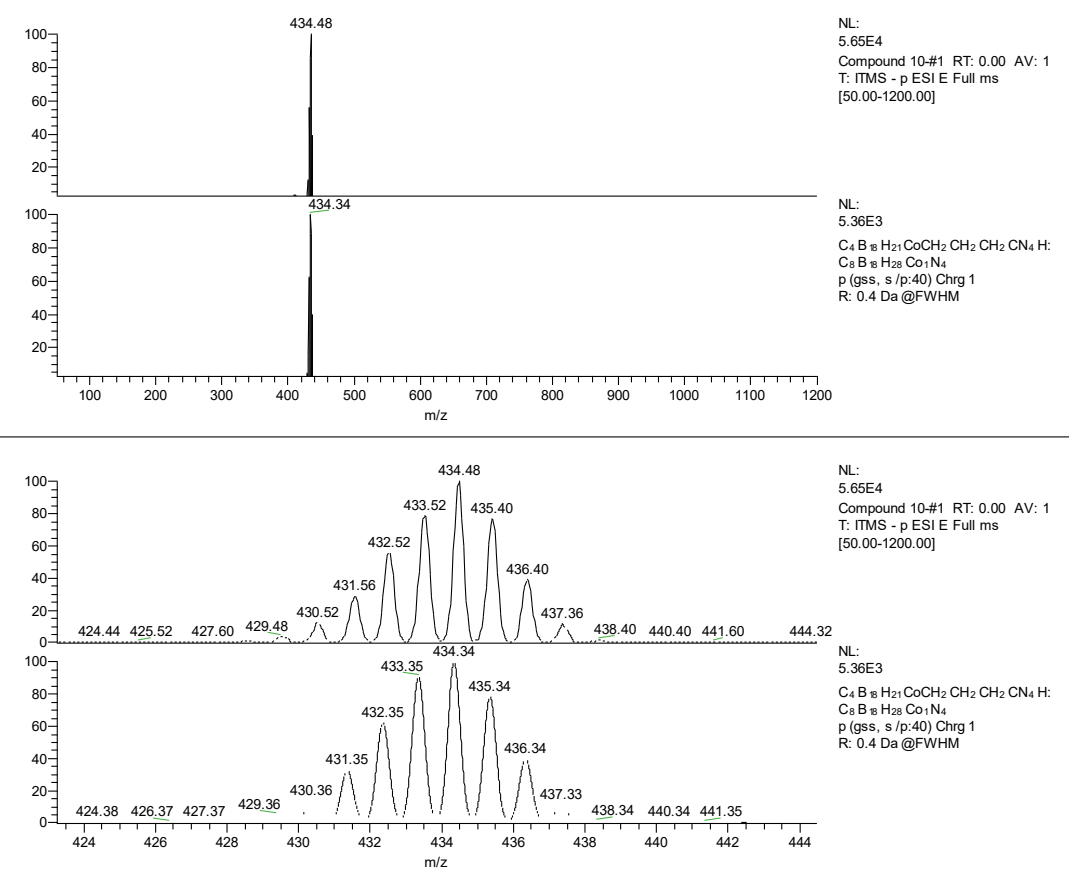

Figure S29. MS of compound $\mathbf{1 0}^{-}$

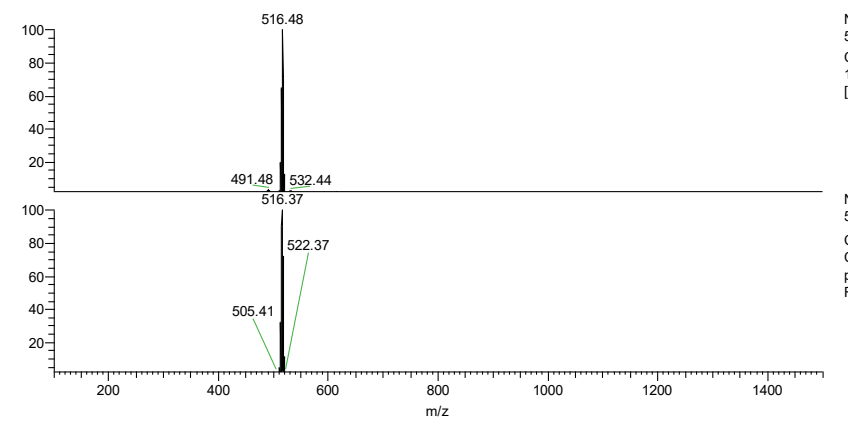

NL:
5.22E3

Compound 11\#1 RT: $0.00 \mathrm{AV}$

[100.00-1500.00]

NL:

$\mathrm{C}_{4} \mathrm{~B}_{8} \mathrm{H}_{20} \mathrm{Co}\left(\mathrm{CH}_{2} \mathrm{CH}_{2} \mathrm{CN}_{4} \mathrm{H}\right)_{2}$

(gss, s/p:40) Chrg

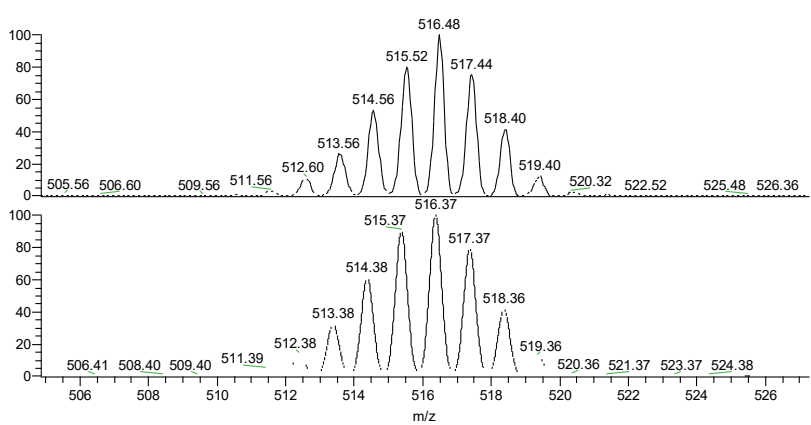

NL:

Compound 11\#1 RT: 0.00 AV:

1 T: ITMS - p ESI E Full ms

[100.00-1500.00]

$\mathrm{NL}:$
$5.35 \mathrm{E} 3$

$\mathrm{C}_{4} \mathrm{~B}_{8} \mathrm{H}_{20} \mathrm{Co}\left(\mathrm{CH}_{2} \mathrm{CH}_{2} \mathrm{CN}_{4} \mathrm{H}_{2}\right.$

p (gss, s/p:40) Chrg

p gss, s/p:40)
R: 0.4 Da @FWHM 1

Figure S30. MS of compound 11 

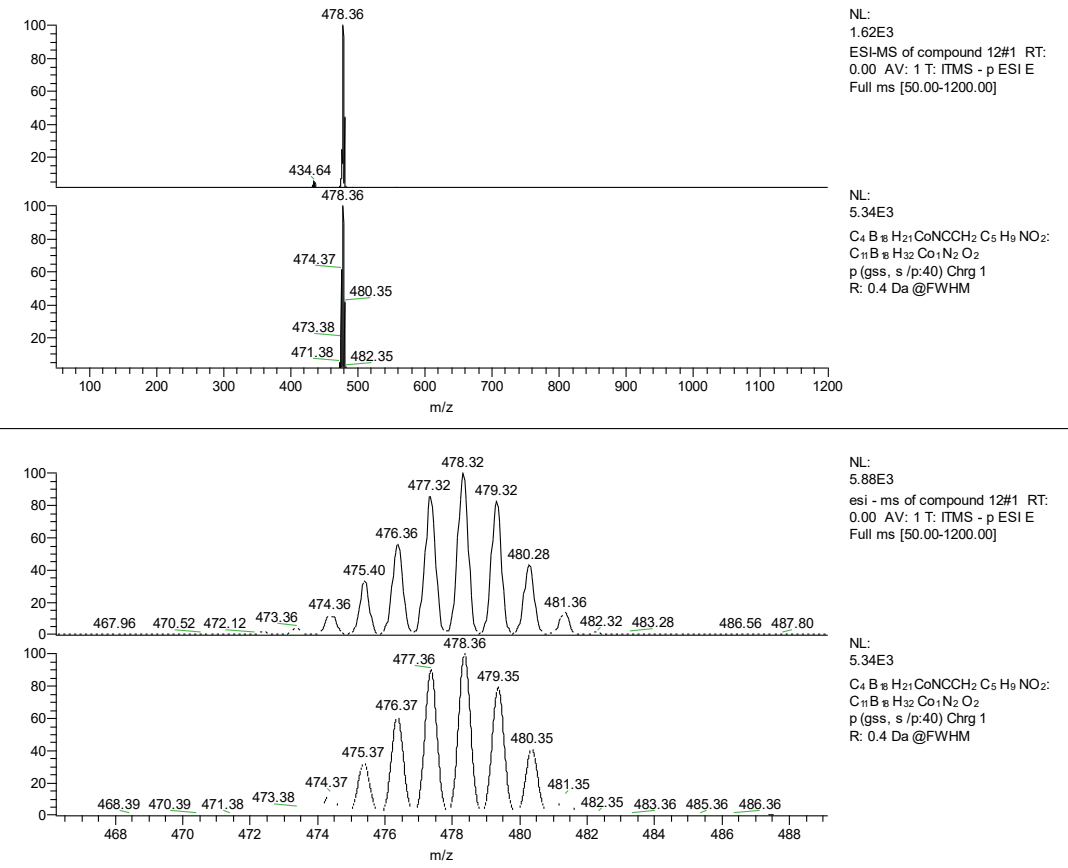

Figure S31. MS of compound 12 